\title{
The census of non-radial pulsation in first-overtone $R R$ Lyrae stars of the OGLE Galactic bulge collection
}

\author{
H. Netzel, ${ }^{1 \star}$ R. Smolec ${ }^{1}$ \\ ${ }^{1}$ Nicolaus Copernicus Astronomical Centre, Polish Academy of Sciences, Bartycka 18, PL-00-716 Warsaw, Poland
}

Accepted XXX. Received YYY; in original form ZZZ

\begin{abstract}
We analyzed photometry for the up-to-date collection of the first-overtone RR Lyrae stars (RRc; 11415 stars) and double-mode RR Lyrae stars (RRd; 148 stars) towards the Galactic bulge from the Optical Gravitational Lensing Experiment. We analyzed frequency spectra of these stars in search for additional, low-amplitude signals, beyond the radial modes. We focused on stars from two groups: $R R_{0.61}$ and $R R_{0.68}$. In the first group, additional low-amplitude signals have periods shorter than the first-overtone period; period ratios fall in the $0.60-0.64$ range. In the second group, additional lowamplitude signals have periods longer than the first-overtone period; period ratios tightly cluster around 0.68. Altogether we have detected 960 and 147 RR Lyrae stars that belong to $R R_{0.61}$ and $R R_{0.68}$ groups, respectively, which yield the incidence rates of 8.3 and 1.3 per cent of the considered sample. We discuss statistical properties of RR Lyrae stars with additional periodicities. For $R R_{0.61}$ group we provide strong arguments that additional periodicities are connected to non-radial pulsation modes of degrees $\ell=8$ and $\ell=9$, as proposed by Dziembowski. We have also detected two double-periodic variables, with two close periodicities, similar to RR Lyrae variable V37 in NGC 6362. Properties of these peculiar variables, which may form a new group of double-mode pulsators, are discussed.
\end{abstract}

Key words: stars: horizontal branch - stars: oscillations - stars: variables: RR Lyrae

\section{INTRODUCTION}

Till recently, RR Lyrae stars were considered to be a textbook example of simple classical pulsating stars. Significant majority pulsate in one radial mode. In majority of stars it is the radial fundamental mode (RRab stars). In a smaller number of stars, it is the radial first-overtone mode (RRc stars). We also know double-mode pulsation in the fundamental mode and the first overtone mode (RRd stars) or in the fundamental mode and the second overtone. The latter group is not very numerous, only a few members are known (e.g. Benkő et al. 2010, 2014). Period of pulsations for RR Lyrae stars is in the $0.2-1 \mathrm{~d}$ range. For a long time, the only flaw on this simple picture was the Blazhko effect, which is a quasi-periodic amplitude and/or phase modulation of pulsations observed in a subgroup of RR Lyrae stars. It was discovered more than a hundred years ago (Blazhko 1907). The Blazhko effect is a frequent phenomenon among RRab stars, for which the incidence rate of modulated stars can be as high as 50 per cent (Jurcsik et al. 2014; Benkö et al. 2014; Smolec et al. 2017). The modulation is less frequent in RRc stars. The analysis of the OGLE Galactic bulge RRc stars

^ E-mail: henia@netzel.pl led to the incidence rate of 5.6 per cent (Netzel et al. 2018). The Blazhko effect was also detected in RRd stars (Jurcsik et al. 2014; Smolec et al. 2015; Soszyński et al. 2016).

Analysis of space photometry revealed that in many RR Lyrae stars additional small-amplitude signals are observed, which cannot be due to radial modes. The first new group that seems to appear at unexpected position in the Petersen diagram (i.e. diagram of period ratio versus the longer period) consists of stars in which the radial first-overtone mode dominates (so either RRc or RRd stars). The additional shorter-period and low-amplitude signal forms period ratio around $0.60-0.64$. We denote this group $R R_{0.61}$. This period ratio suggests that the additional mode cannot correspond to radial pulsations. After first discovery of this additional signal in the RRd star AQ Leo (Gruberbauer et al. 2007), it was found in many more RRc and RRd stars from groundbased photometry (see e.g. Olech \& Moskalik 2009; Netzel et al. 2015c; Jurcsik et al. 2015, and references therein) and from space-based photometry (see e.g. Moskalik et al. 2015; Molnár et al. 2015; Kurtz et al. 2016, and references therein). An explanation of the $R R_{0.61}$ group was proposed by Dziembowski (2016). In this scenario, additional signal forming period ratio $0.60-0.64$ is a harmonic of the non-radial mode of moderate degree $(\ell=8,9)$. 
Another interesting group are RRc stars with the additional long-period signal forming period ratio $P_{10} / P_{x}$ around 0.68 with the first-overtone period. This $R R_{0.68}$ group was reported for the first time by Netzel et al. (2015b). Observed period ratio indicates, that the additional signals have period longer than the period of the fundamental mode, which was never detected in these stars. The problem with an explanation of this group was discussed by Dziembowski (2016).

Prudil et al. (2017) reported discovery of another previously unknown group of double-mode RR Lyrae stars with the additional short-period signal of relatively large amplitude, forming period ratios from the range 0.68-0.72. Explanation of the nature of this additional signal is still missing.

The Optical Gravitational Lensing Experiment (OGLE, Udalski et al. 1992) is a large-scale sky variability survey. OGLE regularly monitors the brightness of stars from the Magellanic System, the Galactic bulge and the Galactic disk in $I$ and $V$ filters. Since 2010 the fourth phase (OGLE-IV, Udalski et al. 2015) is ongoing. The main advantages of the OGLE photometry are a long time base, high sampling and very good quality of photometry. Despite the fact, that OGLE is a ground-based project, the long time base together with a numerous sample make OGLE data the perfect source to search for rare phenomena in pulsating stars and to study long-term behavior of pulsations. In the Galactic bulge fields the OGLE collection of variable stars counts over $38000 \mathrm{RR}$ Lyrae stars (Soszyński et al. 2014, 2017). Among them over 27480 are RRab stars, 11415 are RRc stars, and 148 are RRd stars.

We present the analysis of the full sample of firstovertone RR Lyrae stars from the Galactic bulge. Main aim of this work is to provide the complete census of $R R_{0.61}$ and $R R_{0.68}$ stars. Method of the analysis is discussed in Sec. 2 . In Sec. 3 we present an overview of the results, while in Sec. 4 and $5 R R_{0.61}$ and $R R_{0.68}$ stars are discussed in detail. In Sec. 6 we discuss two interesting objects detected additionally during this analysis. Our findings are summarized in Sec. 7 .

\section{DATA AND ANALYSIS}

The data used for analysis were gathered during the fourth phase of the OGLE project, which started in 2010 and is ongoing (Udalski et al. 2015). Eight seasons of observations carried out in 2010-2017 in the Galactic bulge are available for the analysis. We used observations in the $I$ filter only, as these are more numerous than in the $V$ filter. The input sample for the analysis comprises 11415 RRc stars. Because of the numerous sample, the study was largely automatic.

To search for additional periodicities, automatic script, based on Fourier transform and consecutive pre-whitening method, was used. The first step was to fit the dominant frequency and its harmonics to the data. Frequencies detected in the Fourier transform were fitted to the data in the form:

$$
m(t)=A_{0}+\sum A_{k} \sin \left(2 \pi f_{k} t+\phi_{k}\right)
$$

where $f_{k}, A_{k}$ and $\phi_{k}$ are frequencies, amplitudes and phases of peaks detected in the power spectrum.

Only frequencies which fulfilled the criterion $A_{k} / \sigma\left(A_{k}\right)>$
4 were included in the fit, where $A_{k}$ is an amplitude of the signal and $\sigma\left(A_{k}\right)$ is its error.

In the data we often detect strong signals related to trends and non-stationary pulsation, which increase the noise level in the Fourier transform and hamper the detection of additional periodicities. Trend in the power spectrum manifests as signal or power excess in the low frequency range. High fraction of RRc stars have non-stationary dominant periods, which manifest in power spectrum as unresolved power at the position of the dominant frequency after prewhitening. These signals usually result from slow, often irregular pulsation period changes. In order to remove these signals we used a form of time-dependent prewhitening method, which was proposed by Moskalik et al. (2015). For the application of the time-dependent prewhitening to the OGLE data see Netzel et al. (2015a). In most cases, this method was successful in removing these unwanted signals. After applying time-dependent prewhitening with the first-overtone and its harmonics, the automatic procedure proceeded with the search for additional signals.

The search was performed in the following way: the power spectrum was calculated from 0 to 40 cycles per day. The procedure determined the highest signal in this range. If the signal had a signal-to-noise ratio, $\mathrm{S} / \mathrm{N}$, higher than 4 , then it was added to the fit. Otherwise, the analysis was finished. When a new signal was detected, the procedure decided whether it is independent, or it corresponds to a linear frequency combination of the already detected signals. In the latter case, no new independent frequency was introduced to Eq. 1, but appropriate linear combination term. The procedure also excluded signals located very close to integer frequencies, i.e. $f=1,2,3 \mathrm{c} \mathrm{d}^{-1}$ as these are most likely of diurnal origin.

If no new signals were detected in the power spectrum, or the number of independent frequencies reached 16, then the analysis was finished. The last step was to remove outliers with the $4 \sigma$ criterion.

The output of the procedure were frequency lists for all RRc stars. Then we picked those RRc stars, in which the procedure found signals characteristic for interesting groups: $R R_{0.61}$ and $R R_{0.68}$. For $R R_{0.61}$ group we selected those stars, for which period ratio of the additional signal with the first overtone falls into $0.6-0.64$ range. In the case of $R R_{0.68}$ stars, criterion for range of period ratios was $0.66-0.7$. In case of some stars, time-dependent prewhitening did not remove remnant power in the position of trend or the first overtone. Aliases of remaining signals can be misinterpreted as additional signals. Therefore, the last step of the analysis was visual inspection of prewhitened power spectra in frequency range from 0 to $10 \mathrm{~cd}^{-1}$ for selected stars. Dubious cases were analyzed manually.

We detected additional signals in other stars as well, however, they do not form any new group of multi-mode RR Lyrae stars. Moreover, we do not observe combination frequencies in case of these additional signals, which suggests, that they may be due to contamination.

Additionally, we manually analyzed 148 RRd stars from the Galactic bulge. 


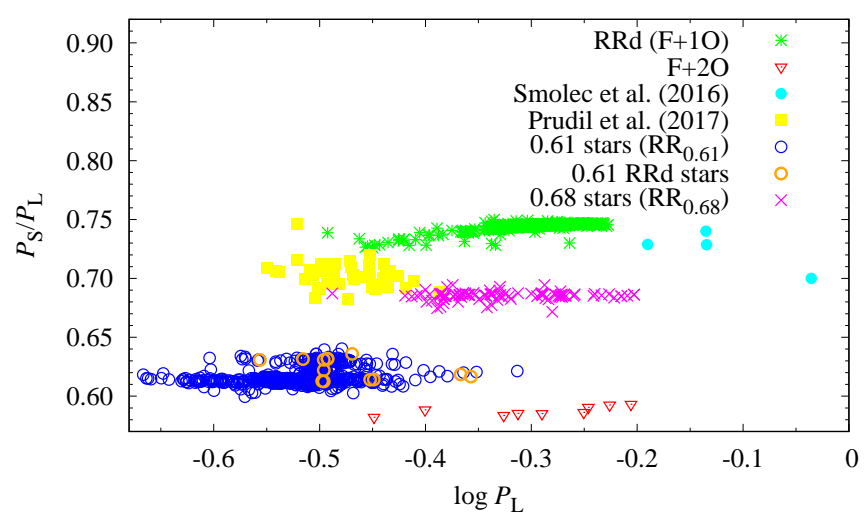

Figure 1. Petersen diagram for multi-mode pulsations in RR Lyrae stars. $R R_{0.61}$ group consists of stars selected during this study and stars reported previously by other studies.

\section{RESULTS}

Analysis of the RRc sample resulted in a discovery of 949 $R R_{0.61}$ stars and $147 R R_{0.68}$ stars. Corresponding incidence rates are 8.3 per cent and 1.3 per cent. Analysis of RRd stars resulted in the detection of $11 R R_{0.61}$ stars and no $R R_{0.68}$ stars. The corresponding incidence rate for RRd $R R_{0.61}$ stars is 7 per cent. List of $R R_{0.61}$ stars with their properties is in Tab. A1, sample of which is presented in Tab. 1 for a reference. Some stars have more than one row in Tab. A1. In these stars we detected more than one signal forming period ratio in the $0.60-0.64$ range. These stars will be discussed in more detail in Sec. 4.2. List of $R R_{0.68}$ with their properties is in Tab. A2, sample of which is presented in Tab. 2. In both tables we provide name of a star, its first-overtone period, period of the additional signal, ratio between two periods, amplitude of the first overtone and ratio of amplitude of the additional signal to the amplitude of the first overtone mode. Last column contains remarks on individual stars.

$R R_{0.61}$ and $R R_{0.68}$ stars are plotted in the Petersen diagram of multi-mode RR Lyrae stars in Fig. $1 . R R_{0.61}$ stars are plotted with blue open circles and RRd stars are plotted with orange open circles. $R R_{0.68}$ stars are plotted with pink crosses. In Sec. 4 and 5 we discuss $R R_{0.61}$ and $R R_{0.68}$ stars in detail. We also detected two interesting stars with additional modes, which are similar to V37 from the NGC 6362 analyzed by Smolec et al. (2017). They are discussed in Sec. 6.

\section{$4 \quad R R_{0.61}$ STARS}

During this analysis of RRc and RRd stars we detected altogether 960 (949 RRc and $11 \mathrm{RRd}$ ) stars falling into the $R R_{0.61}$ group, where 744 stars are new detections (734 RRc and 10 RRd). Combining with previous results from the analysis of the OGLE-III data (Netzel et al. 2015a) and the analysis of two fields from the OGLE-IV data (Netzel et al. 2015c), the total number of $R R_{0.61}$ stars from the OGLE data is $994 \mathrm{RRc}$ and 12 RRd stars.

Several RRc stars detected in the previous studies were not found during this analysis. An example of such star, OGLE-BLG-RRLYR-02077, is plotted in Fig. 2. This star

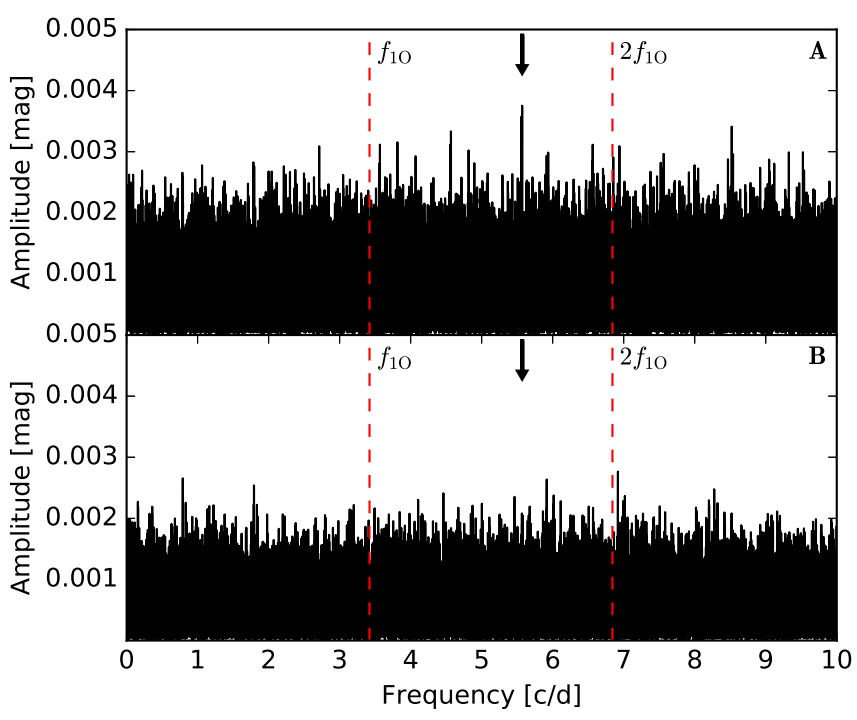

Figure 2. Example of a star, which was detected as $R R_{0.61}$ when O-III data were used (panel A), but was not detected as $R R_{0.61}$ in OGLE-IV data (panel B).

was classified as $R R_{0.61}$ based on the analysis of the OGLEIII data, power spectrum of which is presented in the panel A of Fig. 2. Position of the additional signal is marked with an arrow. In the panel B of Fig. 2 we plotted power spectrum using OGLE-IV data from this analysis. Despite the lower noise level, there is no signal at the position of an arrow. This fact may be a consequence of the variable nature of the additional signal. Netzel et al. (2015c) presented in their fig. 10. analysis of the $R R_{0.61}$ star on the season-by-season basis. Due to variability of the amplitude of the signal, the same star would have different classification depending on the season of observations. Moskalik et al. (2015) in their fig. 7 presented variability in time of the amplitudes of the additional signal and its subharmonics in four $R R_{0.61}$ stars observed by Kepler.

Petersen diagram for $R R_{0.61}$ stars identified in this study is presented in Fig. 3. On the right panel we provided distribution of period ratios. Three sequences are clearly visible in the diagram. Based on minima in the distribution of period ratios we define the borderlines between the sequences. Stars were classified as members of the bottom sequence when period ratio was below 0.62 . For period ratios between 0.62 and 0.625 stars were classified as members of the middle sequence and for period ratios above 0.625 as members of the top sequence. Clearly, the lowest sequence is the most populated and is centered at period ratio 0.613 . The middle sequence is very tight and centered at period ratio 0.622 and the top sequence is centered at period ratio 0.631 with larger scatter than in the middle sequence. By numbers, the bottom, middle and top sequences count 792, 112 and 221 stars, respectively. These numbers do not add up to 960 . This is due to the fact, that in some stars we detected signals corresponding to more than one sequence.

Amplitudes of the additional signals are low, in the mmag range. For the majority of stars, the amplitude is 1-4 mmag, as visible in Fig. 4 which shows the distribution of amplitudes of additional signals for all $R R_{0.61}$ stars. The 
Table 1. Sample table with properties of $R R_{0.61}$ stars. Full table is in the Appendix. Consecutive columns provide name of a star, its first-overtone period, period of the additional signal, ratio between the two periods, amplitude of the first overtone and ratio of amplitude of the additional signal to the amplitude of the first overtone mode. Last column contains remarks on individual stars: 'comb.' - there is a combination between the first overtone and the additional signal, 'bl' - Blazhko effect is present in the star, 'cand.' - additional signal is weak (around $S / N=4$ ) or its daily aliases are higher, $0.5 f_{x}$ - subharmonic of the additonal signal is detected.

\begin{tabular}{lcccccc} 
ID & $P_{1 \mathrm{O}}[\mathrm{d}]$ & $P_{\mathrm{X}}[\mathrm{d}]$ & $P_{\mathrm{X}} / P_{1 \mathrm{O}}$ & $A_{1 \mathrm{O}}[\mathrm{mag}]$ & $A_{\mathrm{X}} / A_{1 \mathrm{O}}$ & Remarks \\
\hline OGLE-BLG-RRLYR-00710 & $0.28749643(7)$ & $0.180509(1)$ & 0.62787 & $0.1420(7)$ & 0.021 & \\
OGLE-BLG-RRLYR-00815 & $0.3117463(3)$ & $0.192034(1)$ & 0.61599 & $0.120(2)$ & 0.041 & \\
OGLE-BLG-RRLYR-01014 & $0.30330353(6)$ & $0.186214(1)$ & 0.61395 & $0.1226(4)$ & 0.022 & \\
OGLE-BLG-RRLYR-01067 & $0.28294809(2)$ & $0.1736623(8)$ & 0.61376 & $0.1426(2)$ & 0.013 & \\
OGLE-BLG-RRLYR-01088 & $0.29471870(5)$ & $0.1811678(9)$ & 0.61471 & $0.1319(4)$ & 0.021 & \\
OGLE-BLG-RRLYR-01097 & $0.2923084(4)$ & $0.179451(1)$ & 0.61391 & $0.1357(5)$ & 0.026
\end{tabular}

Table 2. Sample table with properties of $R R_{0.68}$ stars. Full table is in the Appendix. Consecutive columns provide name of a star, its first-overtone period, period of the additional signal, ratio between the two periods, amplitude of the first overtone and ratio of amplitude of the additional signal to the amplitude of the first overtone mode. Last column contains remarks on individual stars: 'comb.' - there is a combination between the first overtone and the additional signal, 'bl' - Blazhko effect is present in the star.

\begin{tabular}{lcccccc} 
ID & $P_{1 \mathrm{O}}[\mathrm{d}]$ & $P_{\mathrm{X}}[\mathrm{d}]$ & $P_{1 \mathrm{O}} / P_{\mathrm{X}}$ & $A_{1 \mathrm{O}}[\mathrm{mag}]$ & $A_{\mathrm{X}} / A_{1 \mathrm{O}}$ & Remarks \\
\hline OGLE-BLG-RRLYR-01064 & $0.31486155(4)$ & $0.458607(6)$ & 0.68656 & $0.1239(3)$ & 0.016 & \\
OGLE-BLG-RRLYR-01152 & $0.34759513(10)$ & $0.507535(4)$ & 0.68487 & $0.1084(5)$ & 0.04 & comb. \\
OGLE-BLG-RRLYR-01408 & $0.3853534(3)$ & $0.56176(1)$ & 0.68598 & $0.112(1)$ & 0.04 & \\
OGLE-BLG-RRLYR-01436 & $0.3242810(5)$ & $0.47942(2)$ & 0.6764 & $0.120(3)$ & 0.04 & \\
OGLE-BLG-RRLYR-01808 & $0.3161546(2)$ & $0.460225(5)$ & 0.68696 & $0.0937(8)$ & 0.063 & \\
OGLE-BLG-RRLYR-03053 & $0.2898407(1)$ & $0.423478(6)$ & 0.68443 & $0.1273(9)$ & 0.042 & bl
\end{tabular}

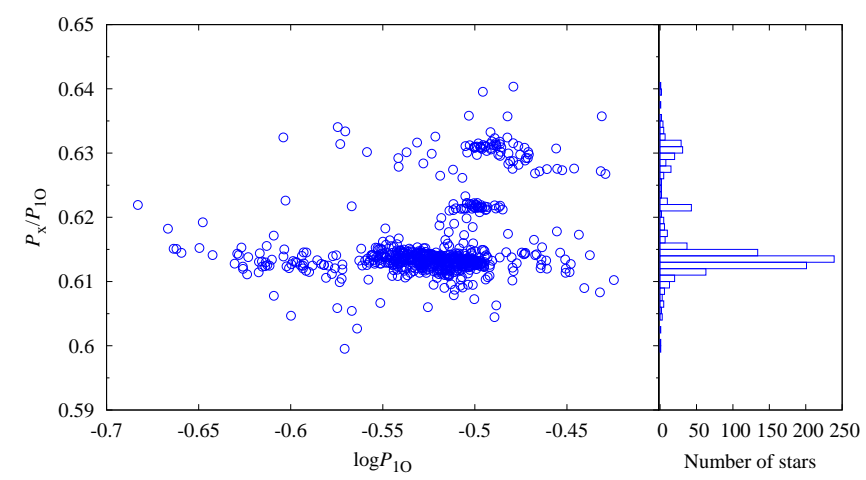

Figure 3. Petersen diagram for RRc and RRd stars with additional 0.61 signal detected in this study. Right panel shows the distribution of period ratios.

highest amplitude is $11 \mathrm{mmag}$, and the lowest is 0.4 mmag. In the bottom panel of Fig. 4 we show the amplitude ratio between the additional mode and the first overtone. For majority of stars this ratio is below 5 per cent, and the peak of the distribution is at 2 per cent. In Fig. 5 we plotted the distribution of amplitudes for the additional signal with the distinction for signals corresponding to the three sequences. Stars with the highest amplitudes are almost only from the lowest sequence. The middle and top sequences correspond to low amplitudes, mostly below 4 mmag.

\subsection{Subharmonics}

In some stars observed by space telescopes, we observe subharmonics of the additional signal centered at the position of $0.5 f_{x}$ and/or $1.5 f_{x}$ (Moskalik et al. 2015). From groundbased data we see almost exclusively signals at $0.5 f_{x}$. Just as
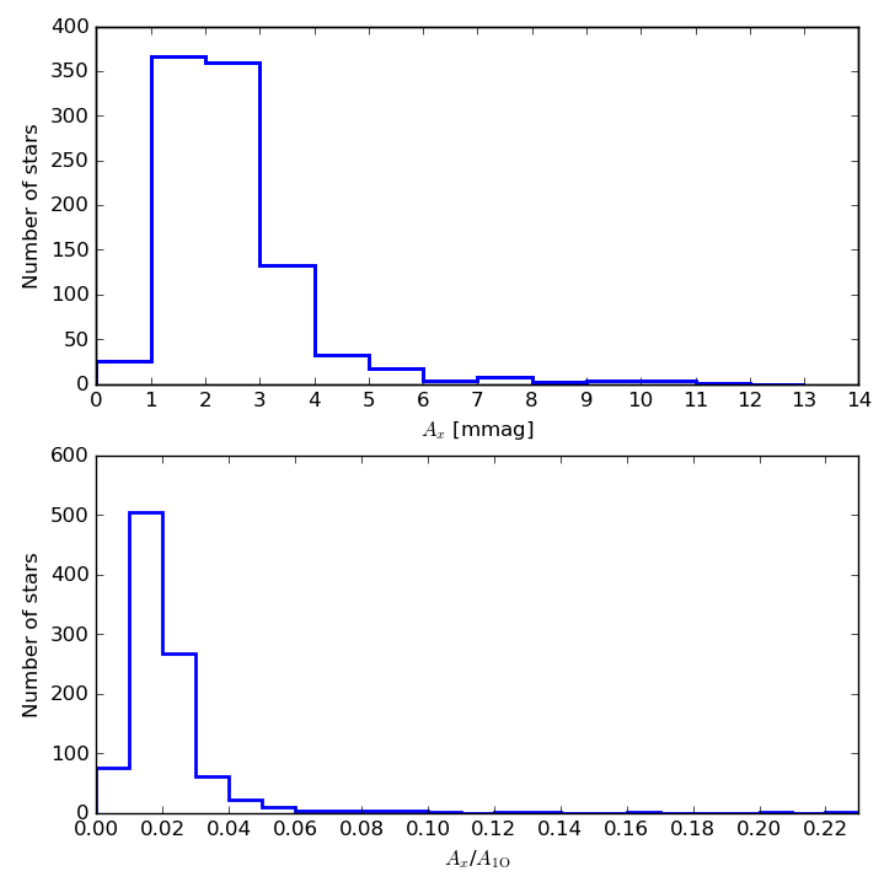

Figure 4. In the top panel we show distribution of amplitudes of the additional signal in $R R_{0.61}$ stars. In the bottom panel we plotted amplitude ratio between the additional signal and the first overtone. One signal for each star included.

signals detected at $f_{x}$, these signals have complex structure. Typically we observe a power excess centered at $0.5 f_{x}$, rather than a single and coherent peak. According to Dziembowski (2016), signals at subharmonic frequencies, $0.5 f_{x}$, are nonradial modes of moderate degrees, $\ell=8$, and $\ell=9$, and the 


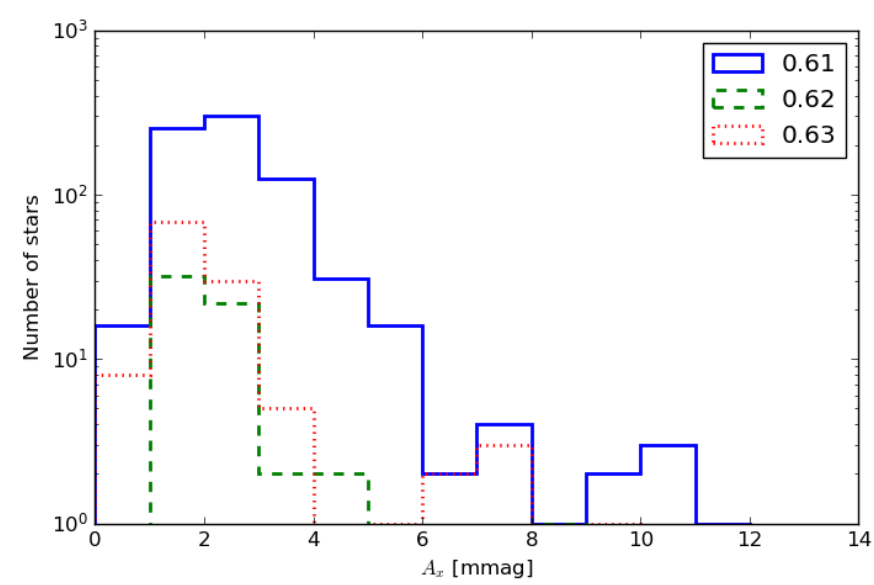

Figure 5. Distribution of amplitudes in $R R_{061}$ stars with distinction to three sequences. One signal for each star included.

signals at $f_{x}$ are harmonics. Due to cancellation effect, it is more difficult to detect the actual non-radial mode, but it is easier to identify its harmonic. Therefore, in the groundbased data, we detect mostly the harmonics, that form the period ratios around 0.6-0.64 with the radial first overtone. We detected signals at $0.5 f_{x}$ for 114 stars from our sample (111 RRc and 3 RRd).

In the model proposed by Dziembowski (2016), the top sequence in the Petersen diagram arises due to harmonics of $\ell=8$ non-radial modes, the bottom sequence arises due to harmonics of $\ell=9$ non-radial modes, and the middle sequence arises due to linear combination frequency of these two modes. Due to differences in cancellation effect for modes with different $\ell$, it should be easier to detect signal corresponding to the non-radial mode with $\ell=8$ than with $\ell=9$. In Fig. 6 we plotted the Petersen diagram for $R R_{0.61}$ stars from our sample. Stars in which we did not detect signal at $0.5 f_{x}$, i.e. the non-radial mode, are plotted with black open circles. Stars in which we detected this signal are plotted with red diamonds. All stars with subharmonic, except eight, correspond to the top sequence, i.e. indeed the nonradial mode of $\ell=8$ is more easily detected than non-radial mode of $\ell=9$. This finding strongly supports the Dziembowski model.

In Fig. 7 we plotted histograms of amplitude ratio between the signal at $0.5 f_{x}$ and the signal at $f_{x}$ (top panel), and between the signal at $0.5 f_{x}$ and the first-overtone mode (bottom panel). Amplitude of the $0.5 f_{x}$ signal constitutes mostly 0.5 to 2 per cent of the amplitude of the first overtone and 60 to 100 per cent of the signal at $f_{x}$. In 35 out of 114 stars we detected higher amplitude the $0.5 f_{x}$ signal than of the $f_{x}$ signal.

In seven stars, which are not members of the $R R_{0.61}$ group, we detected additional signal, which forms period ratio with the first overtone, $P_{10} / P_{y}$ around 0.79 . This additional signal has period longer than the first-overtone period. However, period corresponding to its harmonic, which is not detected in the power spectrum, forms period ratio $0.60-0.64$ with the first overtone. The fact, that in 31 per cent of $R R_{0.61}$ stars in which both signals are detected (35 out of 114) we observe higher amplitude of the signal at $0.5 f_{x}$ than of the

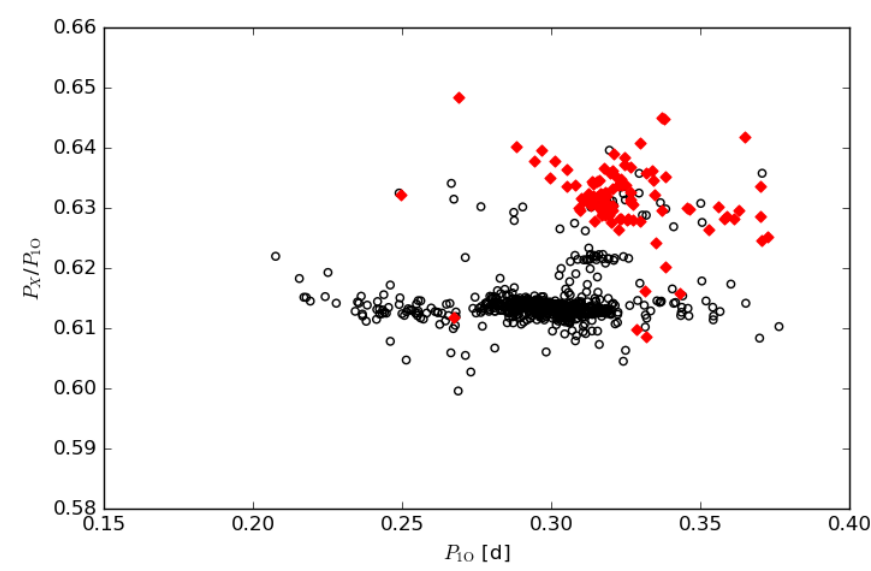

Figure 6. Petersen diagram for OGLE $R R_{0.61}$ stars. Stars without subharmonic in power spectra are plotted with open black circles. For stars in which the signal at the subharmonic frequency is detected, corresponding signal is plotted with red diamonds.
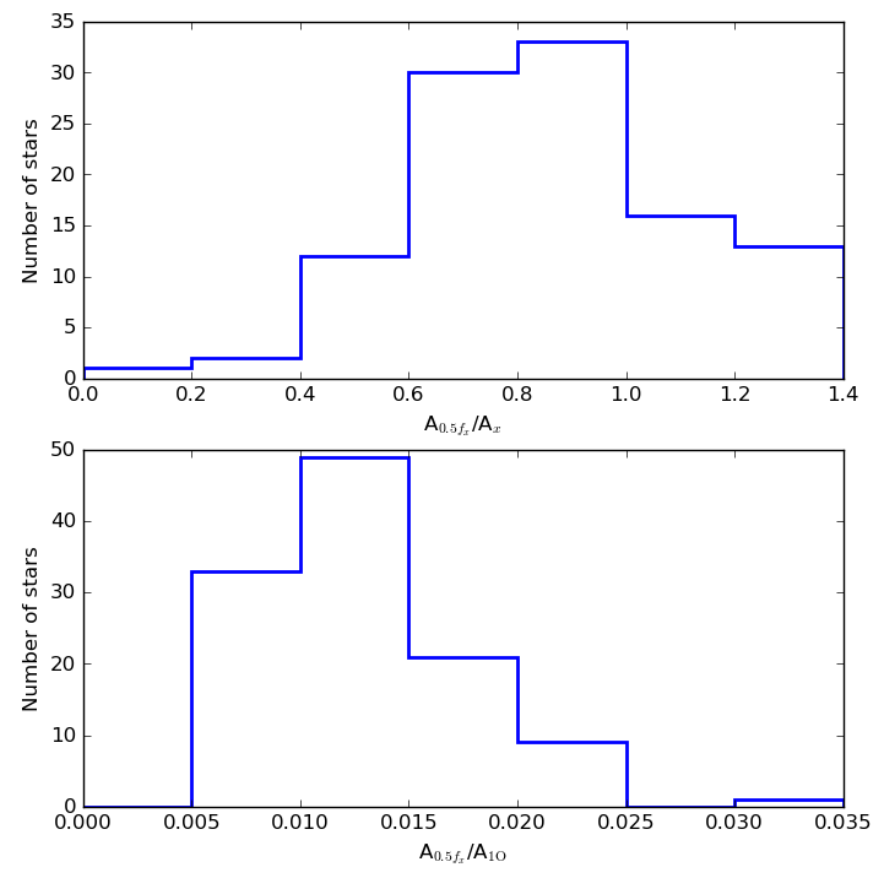

Figure 7. In the top panel we show distribution of amplitude ratio of subharmonic signal to the additional signal. In the bottom panel we plotted amplitude ratio of subharmonic signal to the first overtone.

signal at $f_{x}$ (top panel of Fig. 7) suggests, that these seven stars are in fact $R R_{0.61}$ stars in which we observe only the $0.5 f_{x}$ signal. Properties of these stars are collected in Tab. 3 . In the fourth column of Tab. 3 we provide the ratio of the period corresponding to the harmonic of the detected signal to the first-overtone period. In six out of seven stars this period ratio corresponds to the top sequence, for which it is the most likely to directly detect non-radial mode, i.e. signal corresponding to $0.5 f_{x}$. 
Table 3. Table with properties of stars in which signal fitting into the position of $0.5 f_{\mathrm{x}}$ was detected. Consecutive columns provide ID of a star, period of the first overtone $\left(P_{10}\right)$, and of the detected signal $\left(P_{0.5 f_{\mathrm{x}}}\right)$, period ratio of the harmonic of the detected signal with the first-overtone period $\left(P_{0.5 f_{\mathrm{x}}} /\left(2 P_{10}\right)\right)$, amplitude of the first overtone and amplitude ratio.

\begin{tabular}{lccccc} 
ID & $P_{1 \mathrm{O}}[\mathrm{d}]$ & $P_{0.5 f_{\mathrm{x}}}[\mathrm{d}]$ & $P_{0.5 f_{\mathrm{x}}} /\left(2 P_{1 \mathrm{O}}\right)$ & $A_{1 \mathrm{O}}[\mathrm{mag}]$ & $A_{0.5 f_{\mathrm{x}}} / A_{1 \mathrm{O}}$ \\
\hline OGLE-BLG-RRLYR-02815 & $0.31485827(8)$ & $0.396265(7)$ & 0.62928 & $0.1265(5)$ & 0.017 \\
OGLE-BLG-RRLYR-04622 & $0.3220768(2)$ & $0.407628(7)$ & 0.63281 & $0.1358(9)$ & 0.034 \\
OGLE-BLG-RRLYR-05746 & $0.32689535(6)$ & $0.412324(6)$ & 0.63067 & $0.1284(3)$ & 0.015 \\
OGLE-BLG-RRLYR-06501 & $0.32539511(7)$ & $0.415580(6)$ & 0.63858 & $0.1160(4)$ & 0.014 \\
OGLE-BLG-RRLYR-09926 & $0.38240378(3)$ & $0.471320(4)$ & 0.61626 & $0.1129(2)$ & 0.014 \\
OGLE-BLG-RRLYR-31948 & $0.29931521(4)$ & $0.381558(3)$ & 0.63739 & $0.1277(3)$ & 0.012 \\
OGLE-BLG-RRLYR-32466 & $0.32554603(8)$ & $0.411304(6)$ & 0.63171 & $0.1168(4)$ & 0.016
\end{tabular}

\subsection{Stars with more than one signal}

As it was reported by Netzel et al. (2015c), some stars have more than one signal in the frequency range corresponding to $0.60-0.64$ period ratio. We detected signals corresponding to two sequences in 101 stars from our sample (all classified as RRc) and to three sequences in 34 stars from our sample (33 RRc and $1 \mathrm{RRd)}$. These 34 stars give an excellent opportunity to check whether indeed the middle sequence arises due to linear combination frequency of $\ell=8$ and $\ell=9$ non-radial modes as proposed by Dziembowski (2016).

In this case, the frequency of the signal from the middle sequence $\left(f_{\text {middle }}\right)$ should be an average of the frequencies of the signals from the bottom $\left(f_{0.61}\right)$ and the top $\left(f_{0.63}\right)$ sequences. As these signals vary in time, peaks in the power spectra are very broad (Fig. 8) and merely taking the frequencies of the highest peaks within the power excesses, to test the postulated relation, would lead to non reliable results. Therefore we fitted these excess powers with Gaussian function (plotted with red line in Fig. 8) and adopted the centroid as the frequency of the signals. Green dashed line in Fig. 8 is placed then at $0.5\left(f_{0.61}+f_{0.63}\right)$. Then we constructed the deviation parameter, which we defined as the following frequency difference, $\Delta=\left|f_{\text {middle }}-0.5\left(f_{061}+f_{063}\right)\right|$. In Fig. 9 we show the distribution of the deviation parameter in the discussed sample. The green dashed line corresponds to the adopted resolution of the power spectrum. Its location may suggest that in several stars the discussed relation between position of the three signals is not fulfilled. This is not the case however, as these signals are wide. Frequency spectra of stars in Fig. 8 are sorted by increasing deviation parameter and in the last panel we plotted a star with the largest deviation parameter in the considered sample. It is clear that the calculated deviations are always smaller than full width at half maximum (FWHM) of the fitted Gaussians and the discussed relation is well fulfilled given the nature of the signals.

\section{$5 \quad R R_{0.68}$ STARS}

Stars with additional long-period signals, forming period ratio around 0.68 with the first overtone mode, were detected for the first time in the OGLE data (Netzel et al. 2015b). A few more stars were reported in Netzel \& Smolec (2016). One star, KIC 9453114, is known from the Kepler data (Moskalik et al. 2015). This star belongs to the $R R_{061}$ group and contains additional low frequency signals, one of which places this star also in the $R R_{0.68}$ group. During this study we identified $147 \mathrm{RRc}$ stars with this additional signal. We did not detect this signal in any RRd star. Corresponding incidence rate for RRc stars is 1.3 per cent. 128 stars are new detections. In Fig. 10 we show zoom in the Petersen diagram for all known OGLE $R R_{0.68}$ stars together with KIC 9453114. On the right panel we show distribution of period ratios in these stars. The distribution is centered on period ratio 0.686 , as previously reported by Netzel et al. (2015b). However, there are several outliers with smaller or higher period ratios.

All $R R_{0.68}$ stars detected in this study are collected in Table A2, sample of which is presented in Tab. 2. Consecutive columns provide ID, first-overtone period, period of the additional signal, period ratio, first-overtone amplitude, amplitude ratio of additional signal to the first-overtone mode. Remarks are given in the last column.

We note that contrary to additional signals in $R R_{0.61}$ group, additional variability in $R R_{0.68}$ stars is always coherent. Amplitudes and phases of these signals do not vary in time.

In Fig. 11 we present distribution of amplitudes of the additional signal (top panel) and distribution of amplitude ratio of additional signal to the first overtone (bottom panel). Amplitude of this signal is 2-4 mmag in the majority of stars, which constitutes 1 to 3 per cent of the first overtone amplitude.

Two $R R_{0.68}$ stars are particularly interesting: OGLEBLG-RRLYR-11167 and OGLE-BLG-RRLYR-30601. In OGLE-BLG-RRLYR-11167 we detected two signals corresponding to two period ratios: 0.68271 and 0.68106 . It is the only star which has two rows in the Tab. A2. This star also has the Blazhko effect and was discussed in detail in Netzel et al. (2018). We note that separation between the signals does not correspond to the Blazhko modulation period.

In OGLE-BLG-RRLYR-30601, besides the first overtone and the signal corresponding to the 0.68 period ratio, $f_{0.68}$, we detected one more signal, $f_{x}$, which has period in between the period of the first overtone and the period of the $f_{0.68}$ signal. Both additional signals form combinations with the first overtone. Period ratio between the first overtone and the $f_{x}$ signal is 0.7695 , so higher than for RRd stars. In the Petersen diagram, the two periodicities, $f_{x}$ and $f_{10}$ place OGLE-BLG-RRLYR-30601 at the long-period end of the double-mode sequence of the High Amplitude Delta Scuti (HADS) stars pulsating in the fundamental mode and in the first overtone. If OGLE-BLG-RRLYR-30601 is indeed HADS, then it would show that the $f_{0.68}$ signal can be present in other stars than RR Lyrae (see also Süveges \& Anderson 2018, for the possible detection in Classical Cepheids), and it would be the first star with this peculiar periodicity which pulsates in two radial modes simultaneously. However, clas- 

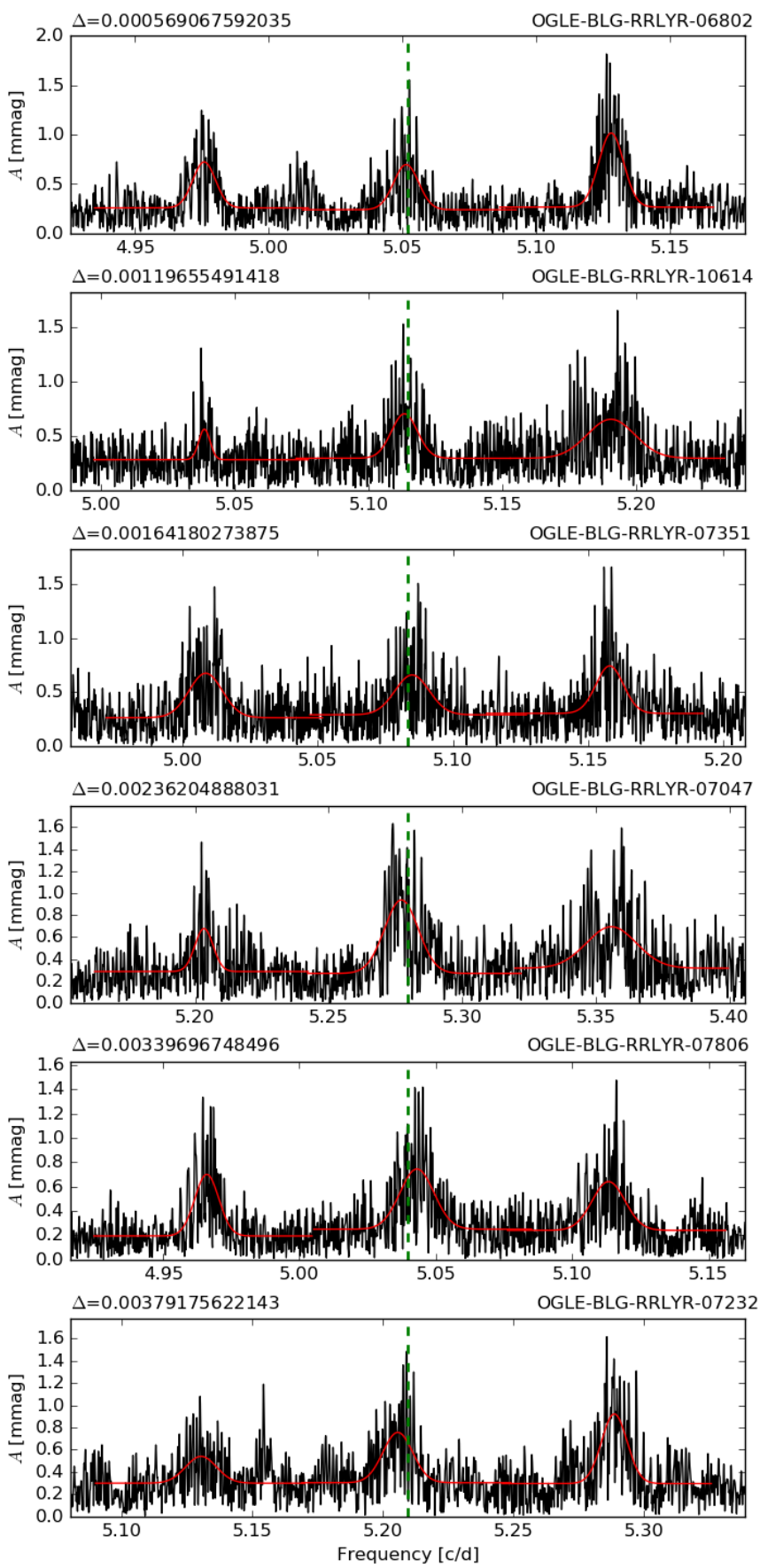

Figure 8. Examples of stars with three signals in the $0.6-0.64$ frequency range. Stars are sorted with increasing $\Delta$ and each correspond to one bin from Fig. 9. Red line shows Gaussian fits to the signals. Green dashed line is placed at $0.5\left(f_{0.61}+f_{0.63}\right)$.

sification of OGLE-BLG-RRLYR-30601 as HADS is not certain, because Fourier decomposition parameters fit very well typical values for RRc stars.

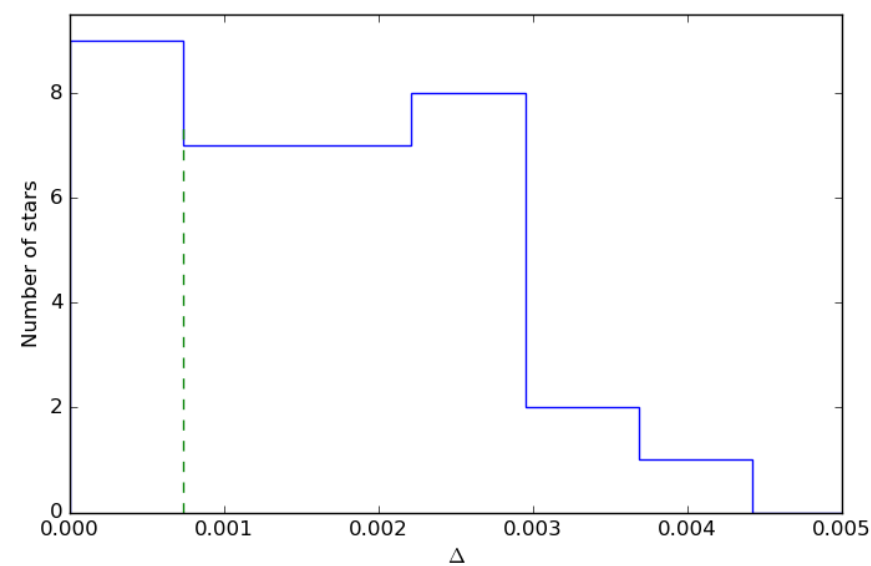

Figure 9. Histogram of the deviation parameter $\Delta=\mid f_{\text {middle }}-$ $0.5\left(f_{061}+f_{063}\right) \mid$ in stars with signals corresponding to three sequences. Green dashed line corresponds to resolution of the power spectrum, which is the same for majority of stars included in the figure.

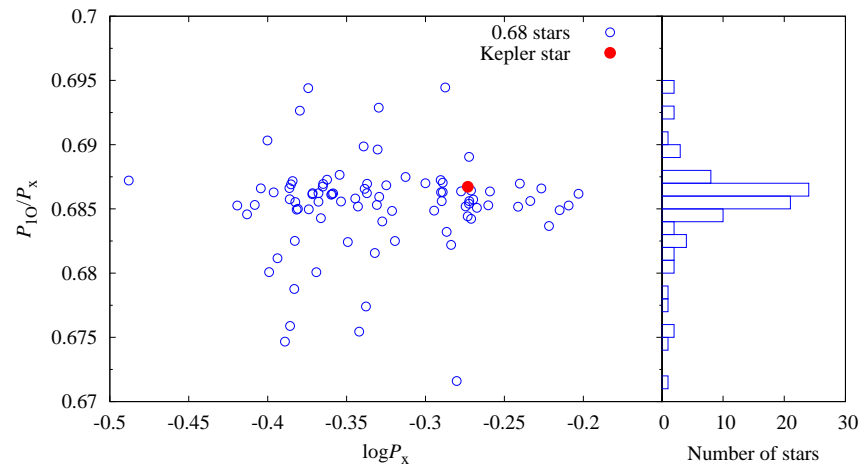

Figure 10. Zoom in the Petersen diagram for $R R_{0.68}$ stars. Stars found in the OGLE data are plotted with blue open circles. Star detected in the Kepler data is plotted with red filled circle. Right panel shows distribution of period ratio among these stars.

\section{OGLE-BLG-RRLYR-11754 AND OGLE-BLG-RRLYR-15059}

We detected two peculiar stars, OGLE-BLG-RRLYR-11754 and OGLE-BLG-RRLYR-15059, which are very similar to RRc star V37 in the globular cluster NGC 6362 analyzed by Smolec et al. (2017). In the period amplitude diagram for RRc stars (Fig. 14) all three variables are located in the short-period and low-amplitude region. Both stars we discuss here have light curves that immediately suggest multiperiodicity or modulation (see top panels of Figs 12 and 13). In Tab. 4 and Tab. 5 we collected all frequencies detected in OGLE-BLG-RRLYR-11754 and OGLE-BLGRRLYR-15059, respectively. Consecutive columns provide frequencies of signals, their amplitudes and phases. Last two columns provide two possible interpretations of the detected signals: modulation or beating. In power spectra of both stars we detected the additional signal with the frequency very close to the frequency of the first overtone. In the modulation scenario this additional signal corresponds to the mod- 

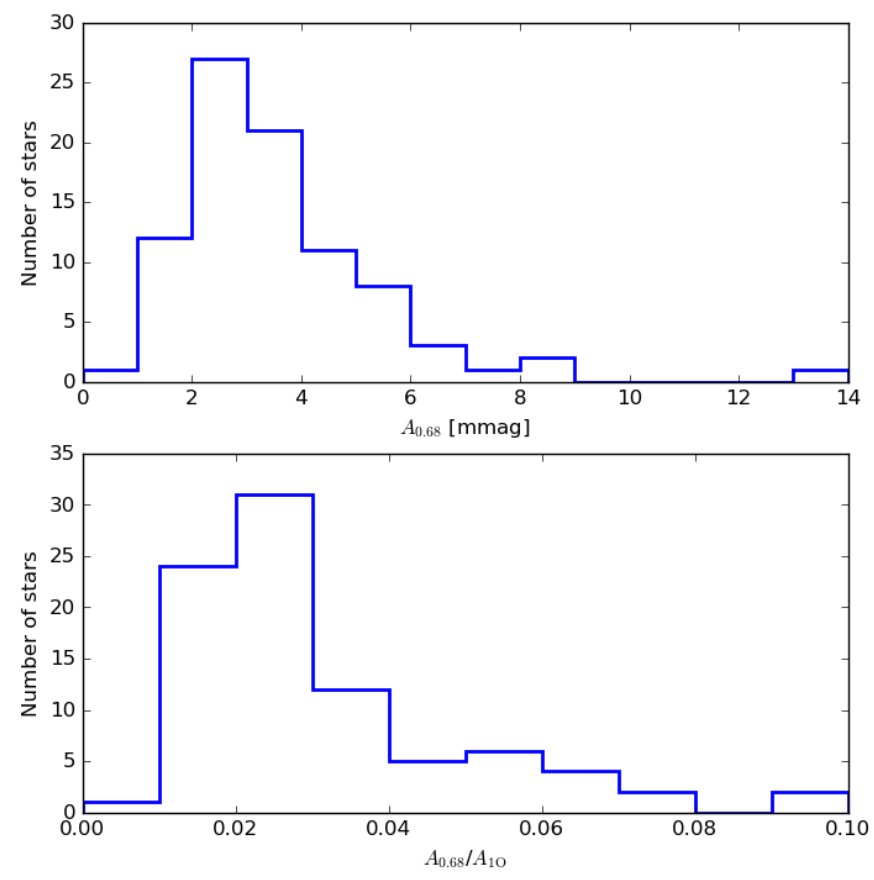

Figure 11. Top panel shows distribution of amplitudes of the additional signal forming period ratio 0.68 with the first overtone. Bottom panel shows distribution of amplitude ratio between the additional signal and the first overtone mode.

ulation sidepeak, and its separation with the first-overtone frequency corresponds to the modulation frequency, $v_{m}$. In the beating scenario this additional signal is treated as an independent frequency and is denoted in both tables as $v_{x}$. As in V37, modulation scenario is unlikely. In the power spectra of both stars we observe very incomplete high-order multiplet components on the lower frequency side of the harmonics of the first overtone. The harmonics, except $2 v_{1}$, are not detected. Such pattern is very different from modulation patterns observed in RRc stars (Netzel et al. 2018). Beating scenario is more realistic for both stars: we observe two close frequencies, their harmonics and linear combinations of the two signals. Period ratio of two signals is 0.9788 and 0.9904 for OGLE-BLG-RRLYR-11754 and OGLE-BLGRRLYR-15059, accordingly, which suggest that at least one signal cannot correspond to the radial mode. In the middle panels of Figs. 12 and 13 we plotted disentangled light curve for the dominant periodicity for OGLE-BLG-RRLYR11754 and OGLE-BLG-RRLYR-15059, respectively. In the bottom panels we present light curves corresponding to the additional lower amplitude signal. In both stars, and in V37 (see fig. 12 in Smolec et al. 2017), light curves corresponding to the dominant periodicity are very symmetric, whereas light curves corresponding to the lower amplitude signal are more non-sinusoidal. We calculated Fourier parameters for light curves corresponding to the dominant and additional periodicity of both stars. Their values are not typical for RRc stars. Especially phase differences are lower than for RRc stars. The nature of all three stars remains a puzzle. We refer the reader to section 3.7 in Smolec et al. (2017) for a more detailed discussion.
Table 4. Two interpretations for OGLE-BLG-RRLYR-11754. First three columns list frequencies, amplitudes and phases of the significant peaks detected in the frequency spectrum of OGLEBLG-RRLYR-11754. The following two columns provide two possible interpretations: 'modulation' and 'beating' scenarios.

\begin{tabular}{cllll}
$v\left(\mathrm{c} \mathrm{d}^{-1}\right)$ & $A(\mathrm{mag})$ & $\phi(\mathrm{rad})$ & modulation? & beating? \\
\hline 0.07989431 & 0.0049 & 0.90 & $v_{\mathrm{m}}$ & $v_{1}-v_{x}$ \\
3.68319790 & 0.0650 & 1.94 & $v_{1}-v_{\mathrm{m}}$ & $v_{x}$ \\
3.76309221 & 0.066343 & 1.26 & $v_{1}$ & $v_{1}$ \\
7.36639581 & 0.009335 & 0.31 & $2 v_{1}-2 v_{\mathrm{m}}$ & $2 v_{x}$ \\
7.44629012 & 0.004961 & 5.70 & $2 v_{1}-v_{\mathrm{m}}$ & $v_{1}+v_{\mathrm{x}}$ \\
7.52618443 & 0.004394 & 4.68 & $2 v_{1}$ & $2 v_{1}$ \\
11.04959371 & 0.004867 & 4.84 & $3 v_{1}-3 v_{\mathrm{m}}$ & $3 v_{x}$ \\
14.73279162 & 0.002674 & 3.21 & $4 v_{1}-4 v_{\mathrm{m}}$ & $4 v_{x}$ \\
\hline
\end{tabular}

Table 5. The same as Tab. 4, but for OGLE-BLG-RRLYR-15059.

\begin{tabular}{cllll}
$v\left(\mathrm{c} \mathrm{d}^{-1}\right)$ & $A(\mathrm{mag})$ & $\phi(\mathrm{rad})$ & modulation? & beating? \\
\hline 3.76105696 & 0.074649 & 4.98 & $v_{1}$ & $v_{1}$ \\
7.52211392 & 0.005627 & 0.24 & $2 v_{1}$ & $2 v_{1}$ \\
3.72483401 & 0.049168 & 3.97 & $v_{1}-v_{\mathrm{m}}$ & $v_{\mathrm{x}}$ \\
7.48589097 & 0.006323 & 4.82 & $2 v_{1}-v_{\mathrm{m}}$ & $v_{1}+v_{\mathrm{x}}$ \\
0.03622295 & 0.003700 & 2.78 & $v_{\mathrm{m}}$ & $v_{1}-v_{x}$ \\
7.44966801 & 0.011156 & 4.48 & $2 v_{1}-2 v_{\mathrm{m}}$ & $2 v_{x}$ \\
11.17450202 & 0.004099 & 4.20 & $3 v_{1}-3 v_{\mathrm{m}}$ & $3 v_{x}$ \\
14.89933602 & 0.003803 & 4.94 & $4 v_{1}-4 v_{\mathrm{m}}$ & $4 v_{x}$ \\
\hline
\end{tabular}

\section{SUMMARY AND CONCLUSIONS}

We analyzed the complete sample of RRc and RRd stars from the Galactic bulge based on the OGLE-IV data. Summary of the results is as follows:

- We have detected $960 R R_{0.61}$ stars, i.e. stars with additional, low-amplitude variability and period ratios, $P_{x} / P_{10}$, in the $0.6-0.64$ range. The incidence rate of this phenomenon, considering the whole OGLE sample is thus 8.3 per cent. The real incidence rate is much higher however. Our previous analysis of selected OGLE-IV fields with the highest observing cadence and hence of relatively low noise points towards incidence rate of 27 per cent (Netzel et al. $2015 \mathrm{c})$. Space photometry, on the other hand, indicates that $R R_{0.61}$ must be common, as nearly all RRc/RRd stars observed from space belong to $R R_{0.61}$ class (Molnár et al. 2015; Moskalik et al. 2015).

- In the Petersen diagram $R R_{0.61}$ stars form three well defined sequences. Bottom sequence is the most populated and is centered at $P_{x} / P_{10}=0.613$. Middle sequence contains lowest number of stars and is centered at $P_{x} / P_{10}=0.622$. The top sequence is not sharply defined. It is centered at $P_{x} / P_{10}=0.631$.

- In $114 R R_{0.61}$ stars, in addition to low amplitude signals at $f_{x}$, we detect signals centered at $0.5 f_{x}$. Of these stars 111 are RRc and 3 are RRd. Signals at $0.5 f_{x}$ correspond to the top sequence in the Petersen diagram for the majority of stars. This finding supports explanation for additional signals proposed by Dziembowski (2016). In this explanation, signals observed at $0.5 f_{x}$ are non-radial modes of $\ell=8$ (top sequence) and $\ell=9$ (bottom sequence). Due to geometric cancellation, modes of $\ell=8$ should be easier to detect and this is what we observe. In this theory, signals detected at $f_{x}$ 

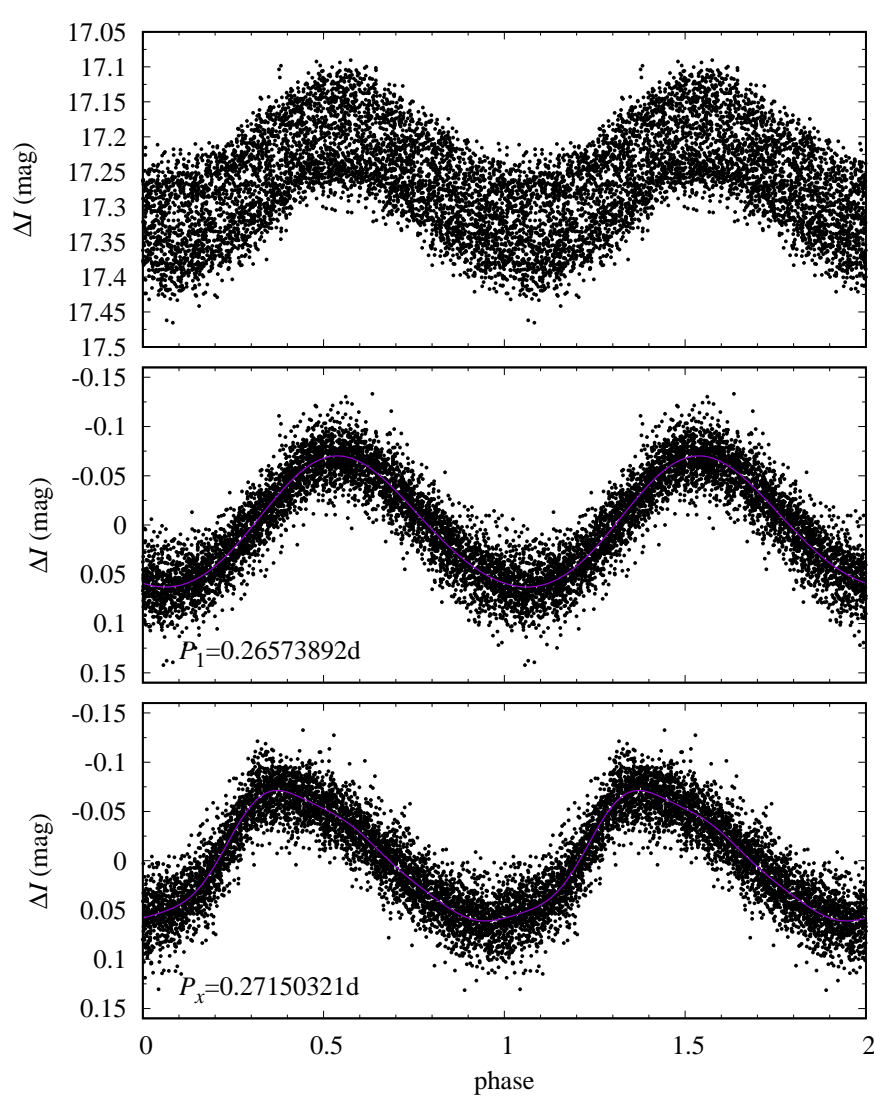

Figure 12. Light curve of OGLE-BLG-RRLYR-11754 phased with the dominant periodicity $\left(P_{1}\right)$ is plotted in the top panel. Disentangled light curves for the dominant and the additional signal are plotted in the middle and bottom panels, respectively.

are harmonics of non-radial modes that do not suffer from geometric cancellation.

- In seven stars we detect additional signals that would fit the expected position of $\ell=8$ non-radial mode. Harmonic is not observed in these stars however.

- In $134 R R_{0.61}$ stars we observe signals corresponding to more than one sequence on the Petersen diagram. 101 stars have signals corresponding to two sequences and 33 stars have signals corresponding to three sequences. According to the model proposed by Dziembowski (2016), the middle sequence in the Petersen diagram arises due to linear combination frequency of the $\ell=8$ and $\ell=9$ modes. For stars in which we detect three signals simultaneously, we confirm that the middle signal is indeed a combination - another point in favor of the Dziembowski model.

- We have detected $147 R R_{0.68}$ stars, i.e. stars with the additional low-amplitude variability and period ratios $P_{10} / P_{x}$ close to 0.686 . The incidence rate is thus 1.3 per cent. 128 stars are new detections. We note that we still lack the interpretation for this mysterious group in which additional signal has period longer that the period of the radial fundamental mode (not detected in these stars).

- We found two RRc stars, similar to V37 from the globular cluster NGC 6362 (Smolec et al. 2017), in which we observe beating of two close frequencies. Dominant frequency has sinusoidal light-curve and the additional period corre-
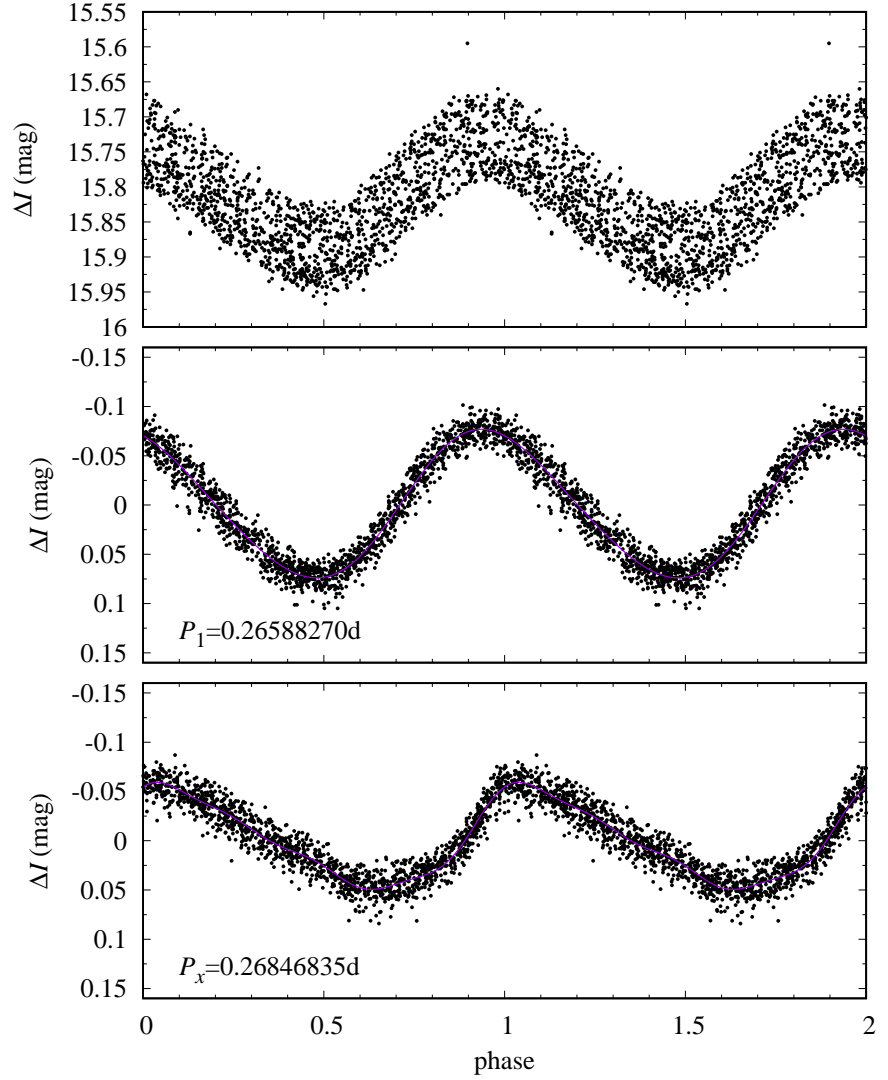

Figure 13. The same as Fig. 12, but for OGLE-BLG-RRLYR15059 .

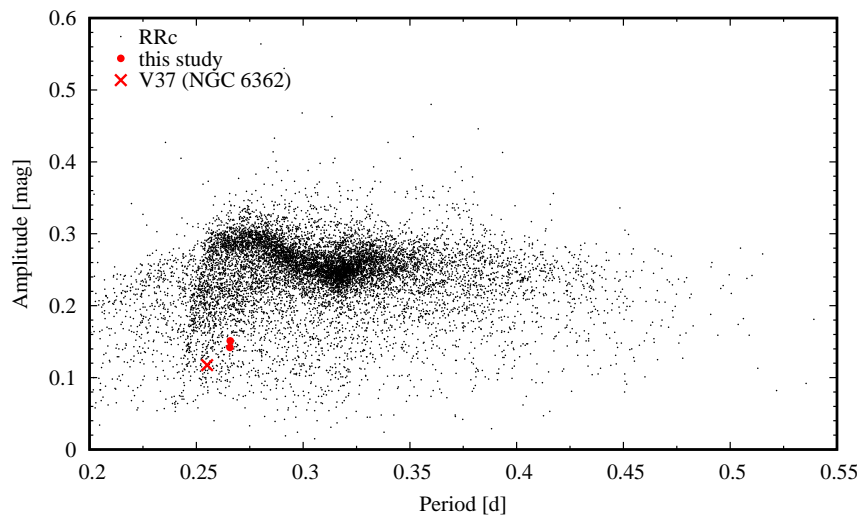

Figure 14. Period - amplitude diagram for RRc stars (black dots). OGLE-BLG-RRLYR-11754 and OGLE-BLG-RRLYR15059 are plotted with red points. V37 star (NGC 6362) is plotted with red cross.

sponds to more non-linear light curve characteristic for pulsations in the fundamental mode. Both light curves differ from typical for RRc stars. As in the case of V37, there is no satisfactory explanation for these stars yet. 


\section{ACKNOWLEDGEMENTS}

This research is supported by the Polish Ministry of Science and Higher Education under grant 0192/DIA/2016/45 within the Diamond Grant Programme for years 2016-2020 (HN) and by the National Science Center, Poland, grant agreement DEC-2015/17/B/ST9/03421 (RS).

\section{REFERENCES}

Benkő J. M., et al., 2010, MNRAS, 409, 1585

Benkő J. M., Plachy E., Szabó R., Molnár L., Kolláth Z., 2014, ApJS, 213, 31

Dziembowski W. A., 2016, Commmunications of the Konkoly Observatory Hungary, 105, 23

Gruberbauer M., et al., 2007, MNRAS, 379, 1498

Jurcsik J., Smitola P., Hajdu G., Nuspl J., 2014, ApJ, 797, L3

Jurcsik J., et al., 2015, ApJS, 219, 25

Kurtz D. W., Bowman D. M., Ebo S. J., Moskalik P., Handberg R., Lund M. N., 2016, MNRAS, 455, 1237

Molnár L., et al., 2015, MNRAS, 452, 4283

Moskalik P., et al., 2015, MNRAS, 447, 2348

Netzel H., Smolec R., 2016, in Różańska A., Bejger M., eds, Vol. 3, 37th Meeting of the Polish Astronomical Society. pp 36-39 (arXiv:1603.05389)

Netzel H., Smolec R., Moskalik P., 2015a, MNRAS, 447, 1173

Netzel H., Smolec R., Dziembowski W., 2015b, MNRAS, 451, L25

Netzel H., Smolec R., Moskalik P., 2015c, MNRAS, 453, 2022

Netzel H., Smolec R., Soszyński I., Udalski A., 2018, MNRAS, 480, 1229

Olech A., Moskalik P., 2009, A\&A, 494, L17

Prudil Z., Smolec R., Skarka M., Netzel H., 2017, MNRAS, 465, 4074

Smolec R., et al., 2015, MNRAS, 447, 3756

Smolec R., Moskalik P., Kałużny J., Pych W., Różyczka M., Thompson I. B., 2017, MNRAS, 467, 2349

Soszyński I., et al., 2014, Acta Astron., 64, 177

Soszyński I., et al., 2016, MNRAS, 463, 1332

Soszyński I., et al., 2017, Acta Astron., 67, 297

Süveges M., Anderson R. I., 2018, MNRAS, 478, 1425

Udalski A., Szymanski M., Kaluzny J., Kubiak M., Mateo M., 1992, Acta Astron., 42, 253

Udalski A., Szymański M. K., Szymański G., 2015, Acta Astron., 65,1

\section{APPENDIX A: DATA TABLES}


Table A1: Properties of $\mathrm{RR}_{0.61}$ stars. Consecutive columns provide name of a star, its first-overtone period, period of the additional signal, ratio between the two periods, amplitude of the first overtone and ratio of amplitude of the additional signal to the amplitude of the first overtone mode. Last column contains remarks on individual stars: 'comb.' - there is a combination between the first overtone and the additional signal, 'bl' - Blazhko effect is present in the star, 'cand.' - additional signal is weak (around $S / N=4$ ) or its daily aliases are higher, $0.5 f_{x}$ - subharmonic of the additional signal is detected.

\begin{tabular}{|c|c|c|c|c|c|c|}
\hline ID & $P_{1 \mathrm{O}}[\mathrm{d}]$ & $P_{\mathrm{X}}[\mathrm{d}]$ & $P_{1 \mathrm{O}} / P_{\mathrm{X}}$ & $A_{10}[\mathrm{mag}]$ & $A_{\mathrm{X}} / A_{1 \mathrm{O}}$ & Remarks \\
\hline OGLE-BLG-RRLYR-00368 & $0.27303168(3)$ & $0.1674370(8)$ & 0.61325 & $0.1013(6)$ & 0.031 & cand. \\
\hline OGLE-BLG-RRLYR-00710 & $0.28749643(7)$ & $0.180509(1)$ & 0.62787 & $0.1420(7)$ & 0.021 & \\
\hline OGLE-BLG-RRLYR-00815 & $0.3117463(3)$ & $0.192034(1)$ & 0.61599 & $0.120(2)$ & 0.041 & \\
\hline OGLE-BLG-RRLYR-01014 & $0.30330353(6)$ & $0.186214(1)$ & 0.61395 & $0.1226(4)$ & 0.022 & \\
\hline OGLE-BLG-RRLYR-01067 & $0.28294809(2)$ & $0.1736623(8)$ & 0.61376 & $0.1426(2)$ & 0.013 & \\
\hline OGLE-BLG-RRLYR-01088 & $0.29471870(5)$ & $0.1811678(9)$ & 0.61471 & $0.1319(4)$ & 0.021 & \\
\hline OGLE-BLG-RRLYR-01097 & $0.2923084(4)$ & $0.179451(1)$ & 0.61391 & $0.1357(5)$ & 0.026 & \\
\hline OGLE-BLG-RRLYR-01099 & $0.25533780(4)$ & $0.1564733(2)$ & 0.61281 & $0.1097(3)$ & 0.036 & \\
\hline OGLE-BLG-RRLYR-01117 & $0.29099566(4)$ & $0.1785419(3)$ & 0.61356 & $0.1356(4)$ & 0.032 & \\
\hline OGLE-BLG-RRLYR-01118 & $0.29082264(4)$ & $0.1786575(9)$ & 0.61432 & $0.1374(3)$ & 0.016 & \\
\hline OGLE-BLG-RRLYR-01183 & $0.30478519(5)$ & $0.186842(1)$ & 0.61303 & $0.1390(4)$ & 0.018 & \\
\hline OGLE-BLG-RRLYR-01186 & $0.30942188(7)$ & $0.190062(1)$ & 0.61425 & $0.1235(4)$ & 0.021 & \\
\hline OGLE-BLG-RRLYR-01209 & $0.31927889(8)$ & $0.195666(1)$ & 0.61284 & $0.1237(5)$ & 0.02 & \\
\hline OGLE-BLG-RRLYR-01213 & $0.25507415(6)$ & $0.1561186(7)$ & 0.61205 & $0.0973(5)$ & 0.035 & \\
\hline OGLE-BLG-RRLYR-01355 & $0.312636(2)$ & $0.191810(2)$ & 0.61352 & $0.123(2)$ & 0.089 & \\
\hline OGLE-BLG-RRLYR-01744 & $0.29585261(6)$ & $0.1810786(9)$ & 0.61206 & $0.1222(4)$ & 0.022 & \\
\hline OGLE-BLG-RRLYR-02119 & $0.24240010(4)$ & $0.1484259(9)$ & 0.61232 & $0.1166(5)$ & 0.019 & \\
\hline OGLE-BLG-RRLYR-02167 & $0.31685902(9)$ & $0.194034(1)$ & 0.61237 & $0.1230(6)$ & 0.022 & \\
\hline OGLE-BLG-RRLYR-02187 & $0.31037026(8)$ & $0.190254(1)$ & 0.61299 & $0.1149(4)$ & 0.023 & cand. \\
\hline OGLE-BLG-RRLYR-02243 & $0.3248316(2)$ & $0.196940(1)$ & 0.60628 & $0.0263(3)$ & 0.042 & \\
\hline OGLE-BLG-RRLYR-02366 & $0.31843153(8)$ & $0.195108(2)$ & 0.61272 & $0.1132(4)$ & 0.018 & \\
\hline OGLE-BLG-RRLYR-02406 & $0.35005209(8)$ & $0.220784(2)$ & 0.63072 & $0.1380(5)$ & 0.017 & \\
\hline OGLE-BLG-RRLYR-02409 & $0.32008133(9)$ & $0.196221(1)$ & 0.61303 & $0.1134(5)$ & 0.023 & \\
\hline \multirow[t]{2}{*}{ OGLE-BLG-RRLYR-02494 } & $0.31476910(10)$ & $0.192830(1)$ & 0.61261 & $0.1126(6)$ & 0.02 & \\
\hline & $0.31476910(10)$ & $0.195666(2)$ & 0.62162 & $0.1126(6)$ & 0.025 & \\
\hline OGLE-BLG-RRLYR-02592 & $0.31349889(8)$ & $0.192323(1)$ & 0.61347 & $0.1223(5)$ & 0.025 & \\
\hline OGLE-BLG-RRLYR-02607 & $0.30212853(5)$ & $0.185281(1)$ & 0.61325 & $0.1299(4)$ & 0.016 & \\
\hline \multirow[t]{2}{*}{ OGLE-BLG-RRLYR-02642 } & $0.33653463(7)$ & $0.212297(2)$ & 0.63083 & $0.1246(4)$ & 0.013 & \\
\hline & $0.33653463(7)$ & $0.211767(2)$ & 0.62926 & $0.1246(4)$ & 0.014 & \\
\hline OGLE-BLG-RRLYR-02963 & $0.30895777(9)$ & $0.189580(1)$ & 0.61361 & $0.1015(5)$ & 0.027 & \\
\hline OGLE-BLG-RRLYR-03124 & $0.30139895(8)$ & $0.1853948(5)$ & 0.61511 & $0.1277(6)$ & 0.027 & cand. \\
\hline OGLE-BLG-RRLYR-03186 & $0.28434260(3)$ & $0.175276(1)$ & 0.61643 & $0.1483(4)$ & 0.011 & \\
\hline OGLE-BLG-RRLYR-03197 & $0.31556218(2)$ & $0.194368(1)$ & 0.61594 & $0.1309(4)$ & 0.014 & \\
\hline OGLE-BLG-RRLYR-03225 & $0.31891007(7)$ & $0.195475(2)$ & 0.61295 & $0.1229(4)$ & 0.02 & \\
\hline OGLE-BLG-RRLYR-03417 & $0.30092069(7)$ & $0.1846212(9)$ & 0.61352 & $0.1273(5)$ & 0.029 & \\
\hline OGLE-BLG-RRLYR-03594 & $0.29519123(7)$ & $0.180908(1)$ & 0.61285 & $0.1379(5)$ & 0.023 & \\
\hline OGLE-BLG-RRLYR-03615 & $0.31293133(6)$ & $0.1944662(9)$ & 0.62143 & $0.1265(4)$ & 0.022 & \\
\hline OGLE-BLG-RRLYR-03687 & $0.2963329(1)$ & $0.181640(1)$ & 0.61296 & $0.0534(4)$ & 0.039 & \\
\hline OGLE-BLG-RRLYR-03710 & $0.3194060(2)$ & $0.204275(2)$ & 0.63955 & $0.1211(10)$ & 0.043 & \\
\hline OGLE-BLG-RRLYR-03797 & $0.26723945(4)$ & $0.163729(1)$ & 0.61267 & $0.1034(3)$ & 0.015 & cand. \\
\hline OGLE-BLG-RRLYR-03952 & $0.28734866(4)$ & $0.1808065(10)$ & 0.62922 & $0.1237(3)$ & 0.013 & \\
\hline OGLE-BLG-RRLYR-03954 & $0.29616868(6)$ & $0.1811923(10)$ & 0.61179 & $0.0598(2)$ & 0.022 & \\
\hline OGLE-BLG-RRLYR-03970 & $0.32661834(9)$ & $0.205836(2)$ & 0.6302 & $0.1232(6)$ & 0.015 & $0.5 f_{x}$ \\
\hline OGLE-BLG-RRLYR-04031 & $0.31144901(6)$ & $0.189695(1)$ & 0.60907 & $0.1271(4)$ & 0.019 & \\
\hline \multirow[t]{2}{*}{ OGLE-BLG-RRLYR-04067 } & $0.3199257(1)$ & $0.195916(1)$ & 0.61238 & $0.1045(5)$ & 0.026 & \\
\hline & $0.3199257(1)$ & $0.199455(1)$ & 0.62344 & $0.1045(5)$ & 0.029 & \\
\hline OGLE-BLG-RRLYR-04083 & $0.28947359(5)$ & $0.177066(1)$ & 0.61168 & $0.1211(3)$ & 0.017 & \\
\hline OGLE-BLG-RRLYR-04105 & $0.30581872(8)$ & $0.187249(1)$ & 0.61229 & $0.1304(5)$ & 0.024 & \\
\hline OGLE-BLG-RRLYR-04144 & $0.3663029(2)$ & $0.223950(2)$ & 0.61138 & $0.1139(10)$ & 0.022 & cand. \\
\hline OGLE-BLG-RRLYR-04209 & $0.28981732(7)$ & $0.177829(4)$ & 0.61359 & $0.1363(6)$ & 0.018 & \\
\hline
\end{tabular}


Table A1 - Continued from previous page

\begin{tabular}{c} 
ID \\
\hline OGLE-BLG-RRLYR-04242 \\
OGLE-BLG-RRLYR-04324 \\
OGLE-BLG-RRLYR-04411 \\
OGLE-BLG-RRLYR-04434 \\
OGLE-BLG-RRLYR-04437 \\
OGLE-BLG-RRLYR-04446 \\
OGLE-BLG-RRLYR-04449 \\
OGLE-BLG-RRLYR-04457 \\
OGLE-BLG-RRLYR-04466 \\
OGLE-BLG-RRLYR-04469 \\
OGLE-BLG-RRLYR-04489 \\
OGLE-BLG-RRLYR-04502 \\
OGLE-BLG-RRLYR-04508 \\
OGLE-BLG-RRLYR-04536 \\
OGLE-BLG-RRLYR-04549 \\
OGLE-BLG-RRLYR-04596 \\
OGLE-BLG-RRLYR-04599 \\
OGLE-BLG-RRLYR-04600 \\
OGLE-BLG-RRLYR-04653 \\
OGLE-BLG-RRLYR-04673 \\
OGLE-BLG-RRLYR-04713 \\
OGLE-BLG-RRLYR-04742
\end{tabular}

OGLE-BLG-RRLYR-04754 OGLE-BLG-RRLYR-04762 OGLE-BLG-RRLYR-04804 OGLE-BLG-RRLYR-04806 OGLE-BLG-RRLYR-04902 OGLE-BLG-RRLYR-04909 OGLE-BLG-RRLYR-04918 OGLE-BLG-RRLYR-04932 OGLE-BLG-RRLYR-04974 OGLE-BLG-RRLYR-04989 OGLE-BLG-RRLYR-05010 OGLE-BLG-RRLYR-05071 OGLE-BLG-RRLYR-05073 OGLE-BLG-RRLYR-05103 OGLE-BLG-RRLYR-05178 OGLE-BLG-RRLYR-05201 OGLE-BLG-RRLYR-05202

OGLE-BLG-RRLYR-05231 OGLE-BLG-RRLYR-05266

OGLE-BLG-RRLYR-05291 OGLE-BLG-RRLYR-05296

OGLE-BLG-RRLYR-05301 OGLE-BLG-RRLYR-05308 OGLE-BLG-RRLYR-05311 OGLE-BLG-RRLYR-05315 OGLE-BLG-RRLYR-05316 OGLE-BLG-RRLYR-05328 OGLE-BLG-RRLYR-05343 OGLE-BLG-RRLYR-05407 OGLE-BLG-RRLYR-05408 OGLE-BLG-RRLYR-05462 OGLE-BLG-RRLYR-05477
$P_{10}[\mathrm{~d}]$

$0.31946328(8)$

$P_{\mathrm{X}}[\mathrm{d}]$

$0.30834017(7)$

$0.29236826(7)$

$0.31633013(8)$

$0.3025502(2)$

$0.30449818(9)$

$0.3657049(1)$

$0.2378579(2)$

$0.29726286(5)$

$0.29726286(5)$

$0.31722366(8)$

$0.29242286(5)$

$0.28919097(6)$

$0.33625015(6)$

$0.31134175(7)$

$0.29963789(6)$

$0.3167623(2)$

$0.28939129(4)$

$0.30773350(6)$

$0.30212857(7)$

$0.29624429(5)$

$0.30986014(9)$

$0.34604132(5)$

$0.34604132(5)$

$0.28631140(4)$

$0.29465508(9)$

$0.31313647(4)$

$0.3221128(1)$

$0.32219135(6)$

$0.31610582(7)$

$0.21911351(4)$

$0.31067201(8)$

$0.29665461(5)$

$0.30669363(5)$

$0.28887291(6)$

$0.33468607(6)$

$0.28573456(6)$

$0.28739704(4)$

$0.37063337(9)$

$0.29692924(5)$

$0.31386605(5)$

$0.31386605(5)$

$0.28600939(2)$

$0.30484525(4)$

$0.30484525(4)$

$0.32072269(2)$

$0.31873205(2)$

$0.31873205(2)$

$0.30561492(3)$

$0.29559922(4)$

$0.29733335(4)$

$0.29958518(4)$

$0.27746606(3)$

$0.30228358(5)$

$0.31876771(9)$

$0.29957178(7)$

$0.32690604(8)$

$0.31296441(10)$ $0.30324951(3)$

$0.195659(1)$

$0.189200(1) \quad 0.61361$

$0.179314(1)$

0.199559(1)

0.187196(1)

$0.186248(2)$

$0.231291(2)$

$0.1453596(9)$

$0.1822009(10)$

$0.184792(1)$

$0.1973855(8)$

$0.1796968(9)$

$0.1777439(10)$

$0.206541(1)$

$0.194946(1)$

$0.1887434(3)$

$0.194071(1)$

$0.177970(1)$

$0.193078(2)$

$0.185288(1)$

$0.1814880(5)$

$0.190247(1)$

$0.217141(1)$

$0.218127(2)$

$0.175781(2)$

0.1806091(7)

$0.192156(2)$

$0.196781(2)$

$0.197265(1)$

$0.193618(1)$

$0.1346378(5)$

$0.1895998(3)$

$0.1816314(7)$

$0.187627(1)$

$0.177289(1)$

$0.210775(2)$

$0.175848(1)$

$0.1765644(9)$

$0.235621(2)$

$0.1822046(9)$

$0.199562(1)$

$0.192226(1)$

$0.1754631(10)$

$0.1869213(9)$

$0.189471(1)$

$0.1969553(10)$

$0.1982157(7)$

$0.1952212(9)$

$0.1872876(5)$

$0.1817250(8)$

$0.1827430(5)$

$0.1839707(9)$

$0.170244(1)$

$0.1847428(5)$

$0.195313(1)$

$0.183965(1)$

$0.204089(2)$

$0.193989(1)$

$0.1858695(4)$
0.61332

0.63086

0.61873

0.61166

0.63245

0.61112

0.61293

0.62165

0.62223

0.61451

0.61462

0.61425

0.62615

0.6299

0.61267

0.61498

0.62742

0.61328

0.61263

0.61398

0.6275

0.63035

0.61395

0.61295

0.61365

0.61091

0.61226

0.61251

0.61447

0.61029

0.61227

0.61177

0.61373

0.62977

0.61542

0.61436

0.63573

0.61363

0.63582

0.61245

0.61349

0.61317

0.62153

0.6141

0.62189

0.61249

0.61282

0.61477

0.61461

0.61408

0.61357

0.61116

0.61271

0.61409

0.6243

0.61984 0.61293
$A_{1 \mathrm{O}}[\mathrm{mag}] \quad A_{\mathrm{X}} / A_{1 \mathrm{O}} \quad$ Remarks

$0.1224(5) \quad 0.018$

$0.1186(5) \quad 0.025$

$0.1404(6) \quad 0.025$

$0.1239(4) \quad 0.021$

$0.139(1) \quad 0.058$

$0.1270(5) \quad 0.025$

$0.1149(4) \quad 0.018$

$0.100(2) \quad 0.029$

$0.1246(3) \quad 0.022$

$0.1246(3) \quad 0.014$

$0.1235(5) \quad 0.016$

$0.1377(5) \quad 0.026$

$0.1390(6) \quad 0.026$

$0.1238(3) \quad 0.017$

$0.1046(4) \quad 0.014$

$0.1224(4) \quad 0.021$

$0.1133(4) \quad 0.024$

$0.1403(3) \quad 0.017$

$0.1416(4) \quad 0.011$

$0.1218(5) \quad 0.026$

$0.1357(4) \quad 0.017$

$0.1356(6) \quad 0.028$

$0.1383(3) \quad 0.015$

$0.1383(3) \quad 0.012$

$0.1275(3) \quad 0.027$

$0.1259(6) \quad 0.046$

$0.1267(6) \quad 0.027$

$0.1127(4) \quad 0.018$

$0.1231(3) \quad 0.019$

$0.1448(5) \quad 0.019$

$0.1039(4) \quad 0.031$

$0.1208(6) \quad 0.022$

$0.1234(4) \quad 0.029$

$0.1307(3) \quad 0.013$

$0.1252(5) \quad 0.018$

$0.1364(3) \quad 0.015$

$0.1080(4) \quad 0.015$

$0.1491(4) \quad 0.016$

$0.1273(4) \quad 0.015$

$0.1282(4) \quad 0.024$

$0.1258(3) \quad 0.017$

$0.1258(3) \quad 0.014$

$0.1443(2) \quad 0.01$

$0.1263(2) \quad 0.016$

$0.1263(2) \quad 0.013$

$0.1146(3) \quad 0.017$

$0.1326(2) \quad 0.02$

$0.1326(2) \quad 0.015$

$0.1240(2) \quad 0.034$

$0.1075(3) \quad 0.018$

$0.1218(2) \quad 0.03$

$0.1317(3) \quad 0.019$

$0.1553(3) \quad 0.011$

$0.1389(3) \quad 0.027$

$0.1131(5) \quad 0.022$

$0.1081(5) \quad 0.022$

$0.1037(2) \quad 0.014$

$0.1210(6) \quad 0.03$

$0.1245(2)$ cand.

$0.5 f_{x}$

$0.5 f_{x}$

$0.5 f_{x}$

$0.5 f_{x}$

$0.5 f_{x}$

cand.

cand. 
Table A1 - Continued from previous page

\begin{tabular}{|c|c|c|c|c|c|c|}
\hline ID & $P_{1 \mathrm{O}}[\mathrm{d}]$ & $P_{\mathrm{X}}[\mathrm{d}]$ & $P_{1 \mathrm{O}} / P_{\mathrm{X}}$ & $A_{1 \mathrm{O}}[\mathrm{mag}]$ & $A_{\mathrm{X}} / A_{1 \mathrm{O}}$ & Remarks \\
\hline \multirow[t]{2}{*}{ OGLE-BLG-RRLYR-05504 } & $0.31484445(5)$ & $0.195837(1)$ & 0.62201 & $0.1220(3)$ & 0.018 & \\
\hline & $0.31484445(5)$ & $0.192942(2)$ & 0.61282 & $0.1220(3)$ & 0.013 & \\
\hline OGLE-BLG-RRLYR-05527 & $0.287153170(10)$ & $0.1762484(7)$ & 0.61378 & $0.1230(3)$ & 0.015 & \\
\hline OGLE-BLG-RRLYR-05529 & $0.34545856(8)$ & $0.217400(2)$ & 0.62931 & $0.1148(4)$ & 0.015 & \multirow{5}{*}{$0.5 f_{x}$, cand. } \\
\hline OGLE-BLG-RRLYR-05531 & $0.30808172(7)$ & $0.1890627(10)$ & 0.61368 & $0.0945(3)$ & 0.026 & \\
\hline OGLE-BLG-RRLYR-05535 & $0.30310723(10)$ & $0.1858008(8)$ & 0.61299 & $0.1178(6)$ & 0.046 & \\
\hline OGLE-BLG-RRLYR-05542 & $0.28751182(2)$ & $0.1773122(6)$ & 0.61671 & $0.1423(1)$ & 0.008 & \\
\hline OGLE-BLG-RRLYR-05600 & $0.30881624(3)$ & $0.1888918(6)$ & 0.61166 & $0.1300(2)$ & 0.024 & \\
\hline OGLE-BLG-RRLYR-05612 & $0.32531345(3)$ & $0.204827(2)$ & 0.62963 & $0.1541(2)$ & 0.006 & \multirow{3}{*}{$\begin{array}{l}0.5 f_{x}, \text { cand. } \\
\text { cand. }\end{array}$} \\
\hline OGLE-BLG-RRLYR-05619 & $0.28861170(4)$ & $0.176206(1)$ & 0.61053 & $0.1434(4)$ & 0.013 & \\
\hline OGLE-BLG-RRLYR-05650 & $0.29725501(4)$ & $0.1828744(9)$ & 0.61521 & $0.1376(4)$ & 0.019 & \\
\hline OGLE-BLG-RRLYR-05666 & $0.30623626(7)$ & $0.187245(1)$ & 0.61144 & $0.1575(5)$ & 0.024 & cand. \\
\hline OGLE-BLG-RRLYR-05672 & $0.28434589(3)$ & $0.1746395(7)$ & 0.61418 & $0.1483(2)$ & 0.013 & \\
\hline OGLE-BLG-RRLYR-05679 & $0.30649533(7)$ & $0.188245(1)$ & 0.61419 & $0.1297(5)$ & 0.024 & \\
\hline \multirow[t]{2}{*}{ OGLE-BLG-RRLYR-05688 } & $0.31810101(6)$ & $0.197472(1)$ & 0.62078 & $0.1240(3)$ & 0.018 & \\
\hline & $0.31810101(6)$ & $0.194848(2)$ & 0.61253 & $0.1240(3)$ & 0.012 & \\
\hline OGLE-BLG-RRLYR-05716 & $0.30570641(5)$ & $0.187377(1)$ & 0.61293 & $0.1287(4)$ & 0.018 & \\
\hline OGLE-BLG-RRLYR-05765 & $0.30791807(5)$ & $0.1889314(8)$ & 0.61358 & $0.1237(3)$ & 0.027 & \\
\hline OGLE-BLG-RRLYR-05770 & $0.30321035(6)$ & $0.186000(1)$ & 0.61344 & $0.1415(5)$ & 0.014 & \\
\hline OGLE-BLG-RRLYR-05784 & $0.29754286(10)$ & $0.182044(1)$ & 0.61182 & $0.1246(7)$ & 0.029 & \multirow[t]{2}{*}{ cand. } \\
\hline OGLE-BLG-RRLYR-05816 & $0.30429030(6)$ & $0.1868125(6)$ & 0.61393 & $0.1188(5)$ & 0.036 & \\
\hline OGLE-BLG-RRLYR-05820 & $0.33503778(9)$ & $0.210846(2)$ & 0.62932 & $0.1206(5)$ & 0.021 & \multirow[t]{5}{*}{$0.5 f_{x}$, cand. } \\
\hline OGLE-BLG-RRLYR-05831 & $0.31680023(4)$ & $0.193866(1)$ & 0.61195 & $0.0927(2)$ & 0.011 & \\
\hline OGLE-BLG-RRLYR-05837 & $0.30154074(3)$ & $0.1851232(4)$ & 0.61392 & $0.1173(2)$ & 0.026 & \\
\hline OGLE-BLG-RRLYR-05895 & $0.30326116(5)$ & $0.185498(1)$ & 0.61168 & $0.1336(4)$ & 0.018 & \\
\hline OGLE-BLG-RRLYR-05896 & $0.26625737(2)$ & $0.161317(1)$ & 0.60587 & $0.1542(2)$ & 0.006 & \\
\hline OGLE-BLG-RRLYR-05898 & $0.32687393(7)$ & $0.206223(1)$ & 0.63089 & $0.1317(4)$ & 0.021 & \multirow[t]{9}{*}{ bl } \\
\hline OGLE-BLG-RRLYR-05924 & $0.29996857(4)$ & $0.1842050(10)$ & 0.61408 & $0.1439(3)$ & 0.017 & \\
\hline OGLE-BLG-RRLYR-05928 & $0.31043474(4)$ & $0.190623(1)$ & 0.61405 & $0.1000(2)$ & 0.012 & \\
\hline \multirow[t]{2}{*}{ OGLE-BLG-RRLYR-05931 } & $0.30140908(4)$ & $0.184753(1)$ & 0.61296 & $0.1343(3)$ & 0.02 & \\
\hline & $0.30140908(4)$ & $0.190245(1)$ & 0.63119 & $0.1343(3)$ & 0.013 & \\
\hline OGLE-BLG-RRLYR-05934 & $0.29263084(3)$ & $0.1794855(7)$ & 0.61335 & $0.1312(2)$ & 0.018 & \\
\hline \multirow[t]{2}{*}{ OGLE-BLG-RRLYR-05937 } & $0.31421047(4)$ & $0.1924421(7)$ & 0.61246 & $0.1243(2)$ & 0.022 & \\
\hline & $0.31421047(4)$ & $0.1952707(10)$ & 0.62146 & $0.1243(2)$ & 0.014 & \\
\hline OGLE-BLG-RRLYR-05956 & $0.2917428(7)$ & $0.179072(2)$ & 0.6138 & $0.1352(4)$ & 0.021 & \\
\hline OGLE-BLG-RRLYR-05965 & $0.31396072(3)$ & $0.1925594(9)$ & 0.61332 & $0.1158(2)$ & 0.018 & $0.5 f_{x}$ \\
\hline \multirow[t]{2}{*}{ OGLE-BLG-RRLYR-05968 } & $0.31756174(6)$ & $0.194676(1)$ & 0.61303 & $0.1112(3)$ & 0.013 & \multirow[t]{2}{*}{$0.5 f_{x}$} \\
\hline & $0.31756174(6)$ & $0.200341(1)$ & 0.63087 & $0.1112(3)$ & 0.013 & \\
\hline OGLE-BLG-RRLYR-05970 & $0.31341714(8)$ & $0.192175(1)$ & 0.61316 & $0.1183(5)$ & 0.023 & $0.5 f_{x}$ \\
\hline OGLE-BLG-RRLYR-05979 & $0.35651736(5)$ & $0.218450(1)$ & 0.61273 & $0.1631(4)$ & 0.013 & \\
\hline OGLE-BLG-RRLYR-05995 & $0.29034905(8)$ & $0.182956(1)$ & 0.63012 & $0.0916(4)$ & 0.027 & \\
\hline OGLE-BLG-RRLYR-06028 & $0.28433564(4)$ & $0.174395(3)$ & 0.61334 & $0.1189(3)$ & 0.014 & \\
\hline OGLE-BLG-RRLYR-06054 & $0.28038913(3)$ & $0.1720319(7)$ & 0.61355 & $0.1431(3)$ & 0.02 & \\
\hline OGLE-BLG-RRLYR-06082 & $0.2732693(1)$ & $0.1673729(7)$ & 0.61248 & $0.1072(4)$ & 0.019 & \multirow[t]{13}{*}{$\mathrm{bl}$} \\
\hline \multirow[t]{2}{*}{ OGLE-BLG-RRLYR-06083 } & $0.30647373(3)$ & $0.1875653(5)$ & 0.61201 & $0.1361(2)$ & 0.021 & \\
\hline & $0.30647373(3)$ & $0.190681(1)$ & 0.62218 & $0.1361(2)$ & 0.008 & \\
\hline OGLE-BLG-RRLYR-06085 & $0.30688310(5)$ & $0.1883120(2)$ & 0.61363 & $0.1258(3)$ & 0.043 & \\
\hline OGLE-BLG-RRLYR-06098 & $0.32589326(4)$ & $0.205831(6)$ & 0.63159 & $0.1367(3)$ & 0.01 & \\
\hline OGLE-BLG-RRLYR-06115 & $0.31080056(9)$ & $0.193109(1)$ & 0.62133 & $0.1176(6)$ & 0.027 & \\
\hline OGLE-BLG-RRLYR-06130 & $0.30383640(4)$ & $0.1863228(8)$ & 0.61323 & $0.0944(2)$ & 0.022 & \\
\hline OGLE-BLG-RRLYR-06143 & $0.30874855(5)$ & $0.1894841(8)$ & 0.61372 & $0.1213(3)$ & 0.023 & \\
\hline OGLE-BLG-RRLYR-06149 & $0.278540310(10)$ & $0.1708440(7)$ & 0.61335 & $0.1050(2)$ & 0.014 & \\
\hline OGLE-BLG-RRLYR-06163 & $0.32623678(6)$ & $0.206107(2)$ & 0.63177 & $0.1302(4)$ & 0.014 & \\
\hline OGLE-BLG-RRLYR-06177 & $0.31831669(9)$ & $0.194100(1)$ & 0.60977 & $0.1299(6)$ & 0.02 & \\
\hline OGLE-BLG-RRLYR-06188 & $0.32420355(7)$ & $0.204654(2)$ & 0.63125 & $0.1186(4)$ & 0.014 & \\
\hline OGLE-BLG-RRLYR-06200 & $0.28698549(2)$ & $0.1757389(5)$ & 0.61236 & $0.1488(2)$ & 0.017 & \\
\hline OGLE-BLG-RRLYR-06226 & $0.26727437(2)$ & $0.167808(2)$ & 0.62785 & $0.1393(2)$ & 0.005 & \multirow[t]{3}{*}{$0.5 f_{x}$, cand. } \\
\hline OGLE-BLG-RRLYR-06238 & $0.23357266(2)$ & $0.1433970(7)$ & 0.61393 & $0.1233(3)$ & 0.012 & \\
\hline OGLE-BLG-RRLYR-06252 & $0.30491824(8)$ & $0.187095(1)$ & 0.61359 & $0.1244(5)$ & 0.026 & \\
\hline
\end{tabular}


Table A1 - Continued from previous page

\begin{tabular}{c} 
ID \\
\hline OGLE-BLG-RRLYR-06265 \\
OGLE-BLG-RRLYR-06268 \\
OGLE-BLG-RRLYR-06273 \\
OGLE-BLG-RRLYR-06329 \\
OGLE-BLG-RRLYR-06346 \\
OGLE-BLG-RRLYR-06349 \\
OGLE-BLG-RRLYR-06352
\end{tabular}

OGLE-BLG-RRLYR-06359 OGLE-BLG-RRLYR-06374 OGLE-BLG-RRLYR-06383 OGLE-BLG-RRLYR-06418 OGLE-BLG-RRLYR-06420 OGLE-BLG-RRLYR-06439 OGLE-BLG-RRLYR-06456 OGLE-BLG-RRLYR-06461

OGLE-BLG-RRLYR-06497 OGLE-BLG-RRLYR-06505 OGLE-BLG-RRLYR-06571 OGLE-BLG-RRLYR-06587 OGLE-BLG-RRLYR-06596 OGLE-BLG-RRLYR-06601 OGLE-BLG-RRLYR-06608 OGLE-BLG-RRLYR-06610

OGLE-BLG-RRLYR-06617 OGLE-BLG-RRLYR-06627 OGLE-BLG-RRLYR-06637 OGLE-BLG-RRLYR-06647 OGLE-BLG-RRLYR-06659 OGLE-BLG-RRLYR-06672 OGLE-BLG-RRLYR-06682 OGLE-BLG-RRLYR-06693 OGLE-BLG-RRLYR-06789 OGLE-BLG-RRLYR-06796 OGLE-BLG-RRLYR-06802

OGLE-BLG-RRLYR-06806 OGLE-BLG-RRLYR-06861 OGLE-BLG-RRLYR-06862 OGLE-BLG-RRLYR-06874 OGLE-BLG-RRLYR-06885 OGLE-BLG-RRLYR-06922

OGLE-BLG-RRLYR-06943 OGLE-BLG-RRLYR-07047

OGLE-BLG-RRLYR-07076 OGLE-BLG-RRLYR-07085 OGLE-BLG-RRLYR-07091 OGLE-BLG-RRLYR-07094

OGLE-BLG-RRLYR-07096 OGLE-BLG-RRLYR-07103 OGLE-BLG-RRLYR-07118

\begin{tabular}{|c|c|c|c|c|c|}
\hline$P_{10}[\mathrm{~d}]$ & $P_{\mathrm{X}}[\mathrm{d}]$ & $P_{1 \mathrm{O}} / P_{\mathrm{X}}$ & $A_{1 \mathrm{O}}[\mathrm{mag}]$ & $A_{\mathrm{X}} / A_{1 \mathrm{O}}$ & Remarks \\
\hline $0.32974362(4)$ & $0.207186(1)$ & 0.62832 & $0.1241(2)$ & 0.011 & $0.5 f_{x}$ \\
\hline $0.33541986(10)$ & $0.206067(2)$ & 0.61436 & $0.1134(5)$ & 0.021 & cand. \\
\hline $0.31513651(7)$ & $0.195710(1)$ & 0.62103 & $0.1189(4)$ & 0.022 & $0.5 f_{x}$, cand. \\
\hline $0.27112592(2)$ & $0.164150(1)$ & 0.60544 & $0.1480(2)$ & 0.007 & \\
\hline $0.2911184(5)$ & $0.1788248(10)$ & 0.61427 & $0.1402(9)$ & 0.019 & \\
\hline $0.30871031(6)$ & $0.189396(1)$ & 0.61351 & $0.1120(3)$ & 0.021 & \\
\hline $0.3162666(2)$ & $0.19393604(7)$ & 0.6132 & $0.1179(2)$ & 0.092 & $0.5 f_{x}$ \\
\hline $0.3162666(2)$ & $0.1996379(6)$ & 0.63123 & $0.1179(2)$ & 0.012 & \\
\hline $0.28202339(3)$ & $0.1731326(5)$ & 0.61389 & $0.1345(3)$ & 0.028 & \\
\hline $0.272961460(10)$ & $0.1672792(7)$ & 0.61283 & $0.1059(3)$ & 0.024 & \\
\hline $0.31355197(7)$ & $0.191110(1)$ & 0.6095 & $0.1359(5)$ & 0.023 & \\
\hline $0.30591874(5)$ & $0.187707(1)$ & 0.61358 & $0.1254(4)$ & 0.016 & cand. \\
\hline $0.31401224(5)$ & $0.1924608(9)$ & 0.61291 & $0.1041(3)$ & 0.023 & \\
\hline $0.30499274(4)$ & $0.186960(2)$ & 0.613 & $0.1232(3)$ & 0.028 & \\
\hline $0.29828851(4)$ & $0.1825150(9)$ & 0.61187 & $0.1167(3)$ & 0.018 & \\
\hline $0.29650769(3)$ & $0.1863225(9)$ & 0.62839 & $0.1303(2)$ & 0.01 & $0.5 f_{x}$ \\
\hline $0.29650769(3)$ & $0.187160(1)$ & 0.63121 & $0.1303(2)$ & 0.01 & \\
\hline $0.281185110(10)$ & $0.1723065(5)$ & 0.61279 & $0.1544(3)$ & 0.024 & \\
\hline $0.3019304(1)$ & $0.1851770(9)$ & 0.61331 & $0.1084(7)$ & 0.012 & \\
\hline $0.29408221(3)$ & $0.1802997(8)$ & 0.61309 & $0.1187(2)$ & 0.014 & \\
\hline $0.37232653(6)$ & $0.233357(1)$ & 0.62675 & $0.1011(2)$ & 0.021 & $0.5 f_{x}$ \\
\hline $0.29223285(2)$ & $0.179622(1)$ & 0.61465 & $0.1369(2)$ & 0.012 & \\
\hline $0.30276894(7)$ & $0.1857690(5)$ & 0.61357 & $0.1221(5)$ & 0.02 & \\
\hline $0.30187600(3)$ & $0.185138(1)$ & 0.61329 & $0.1376(2)$ & 0.014 & \\
\hline $0.3162340(1)$ & $0.196523(2)$ & 0.62145 & $0.1153(2)$ & 0.011 & $0.5 f_{x}$ \\
\hline $0.3162340(1)$ & $0.199508(2)$ & 0.63089 & $0.1153(2)$ & 0.01 & \\
\hline $0.32632516(3)$ & $0.2060354(6)$ & 0.63138 & $0.1379(2)$ & 0.012 & $0.5 f_{x}$ \\
\hline $0.29820477(3)$ & $0.1807152(6)$ & 0.60601 & $0.1154(2)$ & 0.018 & \\
\hline $0.29090046(4)$ & $0.1784261(8)$ & 0.61336 & $0.1384(4)$ & 0.017 & \\
\hline $0.31113207(6)$ & $0.1908536(9)$ & 0.61342 & $0.1210(4)$ & 0.026 & \\
\hline $0.28984257(3)$ & $0.1769863(8)$ & 0.61063 & $0.1155(2)$ & 0.015 & \\
\hline $0.25473335(7)$ & $0.1560260(9)$ & 0.61251 & $0.0728(4)$ & 0.03 & \\
\hline $0.31607422(3)$ & $0.199381(1)$ & 0.6308 & $0.1162(2)$ & 0.009 & \\
\hline $0.29234241(5)$ & $0.1793632(7)$ & 0.61354 & $0.1338(5)$ & 0.011 & \\
\hline $0.24593153(6)$ & $0.1517751(8)$ & 0.61714 & $0.0723(3)$ & 0.024 & \\
\hline $0.33183560(6)$ & $0.212490(1)$ & 0.64035 & $0.1314(3)$ & 0.012 & $0.5 f_{x}$ \\
\hline $0.31830253(5)$ & $0.1950695(9)$ & 0.61284 & $0.1097(2)$ & 0.018 & $0.5 f_{x}$ \\
\hline $0.31830253(5)$ & $0.1979170(8)$ & 0.62179 & $0.1097(2)$ & 0.014 & \\
\hline $0.31830253(5)$ & $0.2010085(10)$ & 0.6315 & $0.1097(2)$ & 0.012 & \\
\hline $0.3100266(1)$ & $0.1904697(7)$ & 0.61437 & $0.1237(9)$ & 0.023 & \\
\hline $0.28913263(3)$ & $0.1774095(6)$ & 0.61359 & $0.1429(3)$ & 0.023 & \\
\hline $0.30842815(4)$ & $0.188368(1)$ & 0.61074 & $0.1361(3)$ & 0.012 & cand. \\
\hline $0.26189420(4)$ & $0.1604280(6)$ & 0.61257 & $0.1145(3)$ & 0.031 & \\
\hline $0.28524591(2)$ & $0.1752734(6)$ & 0.61446 & $0.1501(2)$ & 0.013 & \\
\hline $0.30877929(3)$ & $0.1918750(9)$ & 0.6214 & $0.1288(2)$ & 0.016 & \\
\hline $0.30877929(3)$ & $0.1891884(10)$ & 0.6127 & $0.1288(2)$ & 0.014 & \\
\hline $0.30877929(3)$ & $0.194793(1)$ & 0.63085 & $0.1288(2)$ & 0.01 & \\
\hline $0.32633872(4)$ & $0.207176(2)$ & 0.63485 & $0.1349(2)$ & 0.008 & cand. \\
\hline $0.30496436(2)$ & $0.1865778(8)$ & 0.6118 & $0.1336(2)$ & 0.012 & \\
\hline $0.30496436(2)$ & $0.1893182(9)$ & 0.62079 & $0.1336(2)$ & 0.012 & \\
\hline $0.30496436(2)$ & $0.1922192(9)$ & 0.6303 & $0.1336(2)$ & 0.011 & \\
\hline $0.29638068(2)$ & $0.1815819(9)$ & 0.61266 & $0.1253(2)$ & 0.011 & \\
\hline $0.2919151(2)$ & $0.1804117(6)$ & 0.61803 & $0.117(1)$ & 0.047 & $\mathrm{bl}$ \\
\hline $0.27902722(4)$ & $0.1709779(6)$ & 0.61276 & $0.1472(4)$ & 0.016 & \\
\hline $0.26796838(3)$ & $0.1641731(7)$ & 0.61266 & $0.1094(2)$ & 0.021 & \\
\hline $0.26796838(3)$ & $0.1662106(7)$ & 0.62026 & $0.1094(2)$ & 0.016 & \\
\hline $0.31879405(3)$ & $0.1954778(6)$ & 0.61318 & $0.1165(2)$ & 0.02 & \\
\hline $0.30453247(4)$ & $0.1860722(5)$ & 0.61101 & $0.1236(2)$ & 0.018 & \\
\hline $0.31577711(3)$ & $0.1964602(10)$ & 0.62215 & $0.1212(2)$ & 0.013 & \\
\hline
\end{tabular}

Continued on next page 
Table A1 - Continued from previous page

\begin{tabular}{c} 
ID \\
\hline OGLE-BLG-RRLYR-07163 \\
OGLE-BLG-RRLYR-07232 \\
OGLE-BLG-RRLYR-07265 \\
OGLE-BLG-RRLYR-07292 \\
OGLE-BLG-RRLYR-07303
\end{tabular}

OGLE-BLG-RRLYR-07351

OGLE-BLG-RRLYR-07353

OGLE-BLG-RRLYR-07358 OGLE-BLG-RRLYR-07375

OGLE-BLG-RRLYR-07423 OGLE-BLG-RRLYR-07448 OGLE-BLG-RRLYR-07486 OGLE-BLG-RRLYR-07487 OGLE-BLG-RRLYR-07500

OGLE-BLG-RRLYR-07508 OGLE-BLG-RRLYR-07517 OGLE-BLG-RRLYR-07518 OGLE-BLG-RRLYR-07556 OGLE-BLG-RRLYR-07559 OGLE-BLG-RRLYR-07564 OGLE-BLG-RRLYR-07592 OGLE-BLG-RRLYR-07599

OGLE-BLG-RRLYR-07613 OGLE-BLG-RRLYR-07665

OGLE-BLG-RRLYR-07677 OGLE-BLG-RRLYR-07701 OGLE-BLG-RRLYR-07710 OGLE-BLG-RRLYR-07714 OGLE-BLG-RRLYR-07722 OGLE-BLG-RRLYR-07723 OGLE-BLG-RRLYR-07741 OGLE-BLG-RRLYR-07764

OGLE-BLG-RRLYR-07781 OGLE-BLG-RRLYR-07783 OGLE-BLG-RRLYR-07788 OGLE-BLG-RRLYR-07803

OGLE-BLG-RRLYR-07806

OGLE-BLG-RRLYR-07831

OGLE-BLG-RRLYR-07832 OGLE-BLG-RRLYR-07836 OGLE-BLG-RRLYR-07857 OGLE-BLG-RRLYR-07863 OGLE-BLG-RRLYR-07887 OGLE-BLG-RRLYR-07903

\begin{tabular}{|c|c|c|c|c|c|}
\hline$P_{1 \mathrm{O}}[\mathrm{d}]$ & $P_{\mathrm{X}}[\mathrm{d}]$ & $P_{1 \mathrm{O}} / P_{\mathrm{X}}$ & $A_{10}[\mathrm{mag}]$ & $A_{\mathrm{X}} / A_{1 \mathrm{O}}$ & Remarks \\
\hline $0.31555022(3)$ & $0.193328(1)$ & 0.61267 & $0.1178(2)$ & 0.013 & \\
\hline $0.30926643(3)$ & $0.1891913(9)$ & 0.61174 & $0.1448(2)$ & 0.012 & $0.5 f_{x}$ \\
\hline $0.30926643(3)$ & $0.191975(1)$ & 0.62074 & $0.1448(2)$ & 0.009 & \\
\hline $0.30926643(3)$ & $0.194931(2)$ & 0.6303 & $0.1448(2)$ & 0.007 & \\
\hline $0.28097455(2)$ & $0.170457(1)$ & 0.60666 & $0.1191(3)$ & 0.011 & \\
\hline $0.32492761(2)$ & $0.205060(1)$ & 0.63109 & $0.1302(1)$ & 0.011 & $0.5 f_{x}$ \\
\hline $0.32196111(4)$ & $0.200574(1)$ & 0.62298 & $0.1225(2)$ & 0.011 & cand. \\
\hline $0.32196111(4)$ & $0.197264(1)$ & 0.6127 & $0.1225(2)$ & 0.009 & \\
\hline $0.31626603(4)$ & $0.196576(1)$ & 0.62155 & $0.1194(2)$ & 0.013 & $0.5 f_{x}$ \\
\hline $0.31626603(4)$ & $0.1940850(6)$ & 0.61368 & $0.1194(2)$ & 0.011 & \\
\hline $0.31626603(4)$ & $0.1995293(6)$ & 0.63089 & $0.1194(2)$ & 0.011 & \\
\hline $0.30100588(4)$ & $0.190408(1)$ & 0.63257 & $0.1240(3)$ & 0.011 & $0.5 f_{x}$ \\
\hline $0.30100588(4)$ & $0.189531(2)$ & 0.62966 & $0.1240(3)$ & 0.01 & \\
\hline $0.28948631(7)$ & $0.1775350(9)$ & 0.61328 & $0.1357(6)$ & 0.029 & \\
\hline $0.32464996(4)$ & $0.2017260(8)$ & 0.62136 & $0.1233(2)$ & 0.011 & $0.5 f_{x}$ \\
\hline $0.32464996(4)$ & $0.1989152(8)$ & 0.61271 & $0.1233(2)$ & 0.012 & \\
\hline $0.3288903(2)$ & $0.202127(2)$ & 0.61457 & $0.1136(7)$ & 0.031 & \\
\hline $0.3701437(1)$ & $0.232150(3)$ & 0.62719 & $0.0691(3)$ & 0.025 & $0.5 f_{x}$ \\
\hline $0.31702951(2)$ & $0.1996351(2)$ & 0.62971 & $0.1403(1)$ & 0.013 & \\
\hline $0.31804572(7)$ & $0.195036(1)$ & 0.61323 & $0.1208(4)$ & 0.018 & \\
\hline $0.32307860(2)$ & $0.2041244(10)$ & 0.63181 & $0.1259(1)$ & 0.01 & $0.5 f_{x}$ \\
\hline $0.32307860(2)$ & $0.197871(1)$ & 0.61245 & $0.1259(1)$ & 0.009 & \\
\hline $0.29537403(3)$ & $0.1810988(6)$ & 0.61312 & $0.1356(2)$ & 0.024 & \\
\hline $0.32565035(3)$ & $0.2050801(4)$ & 0.62976 & $0.1219(2)$ & 0.018 & $0.5 f_{x}$ \\
\hline $0.29360651(2)$ & $0.1802886(3)$ & 0.61405 & $0.1356(2)$ & 0.039 & \\
\hline $0.31412516(5)$ & $0.192633(1)$ & 0.61324 & $0.1196(3)$ & 0.016 & $0.5 f_{x}$ \\
\hline $0.32052463(3)$ & $0.202284(1)$ & 0.6311 & $0.1127(1)$ & 0.009 & \\
\hline $0.27633242(7)$ & $0.174135(1)$ & 0.63016 & $0.1122(5)$ & 0.022 & \\
\hline $0.26726043(5)$ & $0.1687514(8)$ & 0.63141 & $0.1046(4)$ & 0.024 & \\
\hline $0.31312320(7)$ & $0.194808(2)$ & 0.62214 & $0.1106(4)$ & 0.015 & \\
\hline $0.31312320(7)$ & $0.192049(1)$ & 0.61333 & $0.1106(4)$ & 0.017 & \\
\hline $0.30348619(7)$ & $0.185701(1)$ & 0.61189 & $0.1309(5)$ & 0.021 & \\
\hline $0.26829993(4)$ & $0.1660553(9)$ & 0.61892 & $0.1051(3)$ & 0.015 & \\
\hline $0.26829993(4)$ & $0.1694032(4)$ & 0.63139 & $0.1051(3)$ & 0.009 & \\
\hline $0.29485394(4)$ & $0.180967(1)$ & 0.61375 & $0.1173(3)$ & 0.021 & \\
\hline $0.29795139(3)$ & $0.1826693(6)$ & 0.61308 & $0.1468(2)$ & 0.015 & \\
\hline $0.26706107(5)$ & $0.162881(1)$ & 0.6099 & $0.0996(4)$ & 0.026 & \\
\hline $0.36977792(3)$ & $0.224940(1)$ & 0.60831 & $0.1251(1)$ & 0.008 & \\
\hline $0.32054475(3)$ & $0.1956329(7)$ & 0.61031 & $0.1522(2)$ & 0.014 & $0.5 f_{x}$ \\
\hline $0.35600180(5)$ & $0.217684(1)$ & 0.61147 & $0.1310(2)$ & 0.01 & $0.5 f_{x}$ \\
\hline $0.30473373(6)$ & $0.1865450(6)$ & 0.61216 & $0.1064(3)$ & 0.035 & \\
\hline $0.30888116(4)$ & $0.1893801(8)$ & 0.61312 & $0.1219(3)$ & 0.021 & \\
\hline $0.30888116(4)$ & $0.194906(1)$ & 0.63101 & $0.1219(3)$ & 0.013 & \\
\hline $0.4291866(3)$ & $0.264511(2)$ & 0.61631 & $0.1036(6)$ & 0.014 & cand. \\
\hline $0.26444657(8)$ & $0.1616242(10)$ & 0.61118 & $0.1547(9)$ & 0.027 & \\
\hline $0.29508748(5)$ & $0.180718(1)$ & 0.61242 & $0.1281(4)$ & 0.014 & \\
\hline $0.31390033(3)$ & $0.1921996(8)$ & 0.61229 & $0.1245(2)$ & 0.021 & \\
\hline $0.31390033(3)$ & $0.198115(1)$ & 0.63114 & $0.1245(2)$ & 0.009 & \\
\hline $0.31899507(3)$ & $0.195456(1)$ & 0.61272 & $0.1233(1)$ & 0.011 & $0.5 f_{x}$ \\
\hline $0.31899507(3)$ & $0.1982080(8)$ & 0.62135 & $0.1233(1)$ & 0.013 & \\
\hline $0.31899507(3)$ & $0.2014255(9)$ & 0.63144 & $0.1233(1)$ & 0.011 & \\
\hline $0.31313989(4)$ & $0.1919759(10)$ & 0.61307 & $0.1168(3)$ & 0.029 & $0.5 f_{x}$ \\
\hline $0.31313989(4)$ & $0.194856(1)$ & 0.62227 & $0.1168(3)$ & 0.011 & \\
\hline $0.25530214(8)$ & $0.1568946(7)$ & 0.61454 & $0.1042(7)$ & 0.014 & \\
\hline $0.32262696(8)$ & $0.203509(1)$ & 0.63079 & $0.1507(6)$ & 0.017 & $0.5 f_{x}$ \\
\hline $0.32087805(3)$ & $0.1993438(6)$ & 0.62124 & $0.1238(2)$ & 0.016 & \\
\hline $0.32476360(9)$ & $0.205016(1)$ & 0.63128 & $0.1263(5)$ & 0.021 & \\
\hline $0.30227798(6)$ & $0.185907(1)$ & 0.61502 & $0.1222(4)$ & 0.016 & \\
\hline $0.25965543(2)$ & $0.1592508(8)$ & 0.61332 & $0.1493(2)$ & 0.008 & \\
\hline
\end{tabular}


Table A1 - Continued from previous page

\begin{tabular}{|c|c|c|c|c|c|c|}
\hline ID & $P_{1 \mathrm{O}}[\mathrm{d}]$ & $P_{\mathrm{X}}[\mathrm{d}]$ & $P_{1 \mathrm{O}} / P_{\mathrm{X}}$ & $A_{1 \mathrm{O}}[\mathrm{mag}]$ & $A_{\mathrm{X}} / A_{1 \mathrm{O}}$ & Remarks \\
\hline OGLE-BLG-RRLYR-07907 & $0.28778891(3)$ & $0.1765896(7)$ & 0.61361 & $0.1455(3)$ & 0.017 & \\
\hline OGLE-BLG-RRLYR-07928 & $0.26103870(5)$ & $0.1650893(7)$ & 0.63243 & $0.0909(3)$ & 0.03 & \\
\hline OGLE-BLG-RRLYR-07937 & $0.28029899(2)$ & $0.1721190(4)$ & 0.61406 & $0.1367(2)$ & 0.066 & \\
\hline \multirow[t]{2}{*}{ OGLE-BLG-RRLYR-07961 } & $0.31580997(4)$ & $0.196416(1)$ & 0.62194 & $0.1197(3)$ & 0.015 & \\
\hline & $0.31580997(4)$ & $0.193709(1)$ & 0.61337 & $0.1197(3)$ & 0.013 & \\
\hline OGLE-BLG-RRLYR-07962 & $0.24462748(3)$ & $0.1498247(5)$ & 0.61246 & $0.1122(3)$ & 0.026 & \\
\hline OGLE-BLG-RRLYR-07964 & $0.32793237(3)$ & $0.1996974(8)$ & 0.60896 & $0.1349(2)$ & 0.013 & cand. \\
\hline OGLE-BLG-RRLYR-07965 & $0.27619564(2)$ & $0.1697181(7)$ & 0.61449 & $0.1473(3)$ & 0.013 & \\
\hline OGLE-BLG-RRLYR-07968 & $0.29742990(3)$ & $0.182261(1)$ & 0.61279 & $0.1189(3)$ & 0.012 & \\
\hline OGLE-BLG-RRLYR-07977 & $0.21697698(3)$ & $0.1334638(7)$ & 0.61511 & $0.1026(4)$ & 0.019 & \\
\hline OGLE-BLG-RRLYR-07996 & $0.31787979(5)$ & $0.197504(1)$ & 0.62132 & $0.1282(3)$ & 0.012 & $0.5 f_{x}$, cand. \\
\hline OGLE-BLG-RRLYR-07999 & $0.326412300(10)$ & $0.2029670(4)$ & 0.62181 & $0.1036(2)$ & 0.018 & $0.5 f_{x}$ \\
\hline \multirow[t]{2}{*}{ OGLE-BLG-RRLYR-08002 } & $0.30879120(3)$ & $0.1891872(8)$ & 0.61267 & $0.1166(2)$ & 0.013 & \\
\hline & $0.30879120(3)$ & $0.1946830(10)$ & 0.63047 & $0.1166(2)$ & 0.009 & \\
\hline OGLE-BLG-RRLYR-08033 & $0.27782677(2)$ & $0.1706193(10)$ & 0.61412 & $0.1831(3)$ & 0.009 & \\
\hline OGLE-BLG-RRLYR-08047 & $0.33708467(5)$ & $0.2120639(3)$ & 0.62911 & $0.1433(3)$ & 0.017 & $0.5 f_{x} \mathrm{bl}$ \\
\hline \multirow{2}{*}{ OGLE-BLG-RRLYR-08048 } & $0.31430279(3)$ & $0.192294(5)$ & 0.61181 & $0.1339(2)$ & 0.012 & $0.5 f_{x}$ \\
\hline & $0.31430279(3)$ & $0.195128(2)$ & 0.62083 & $0.1339(2)$ & 0.008 & \\
\hline \multirow[t]{2}{*}{ OGLE-BLG-RRLYR-08084 } & $0.31469810(5)$ & $0.192996(1)$ & 0.61327 & $0.1279(3)$ & 0.016 & \\
\hline & $0.31469810(5)$ & $0.195619(1)$ & 0.62161 & $0.1279(3)$ & 0.012 & \\
\hline OGLE-BLG-RRLYR-08087 & $0.31632144(7)$ & $0.1939952(7)$ & 0.61329 & $0.1073(4)$ & 0.021 & \\
\hline OGLE-BLG-RRLYR-08097 & $0.31846184(10)$ & $0.198016(2)$ & 0.62179 & $0.1209(5)$ & 0.02 & cand. \\
\hline \multirow[t]{2}{*}{ OGLE-BLG-RRLYR-08108 } & $0.31320005(4)$ & $0.191847(1)$ & 0.61254 & $0.1235(2)$ & 0.012 & \\
\hline & $0.31320005(4)$ & $0.197477(1)$ & 0.63051 & $0.1235(2)$ & 0.011 & \\
\hline OGLE-BLG-RRLYR-08123 & $0.28761001(7)$ & $0.175820(1)$ & 0.61131 & $0.1187(5)$ & 0.01 & cand. \\
\hline OGLE-BLG-RRLYR-08125 & $0.27681123(3)$ & $0.1696468(4)$ & 0.61286 & $0.1011(2)$ & 0.03 & \\
\hline OGLE-BLG-RRLYR-08137 & $0.3079950(10)$ & $0.1890322(7)$ & 0.61375 & $0.1286(4)$ & 0.02 & \\
\hline OGLE-BLG-RRLYR-08138 & $0.31863878(4)$ & $0.201030(1)$ & 0.6309 & $0.1192(3)$ & 0.012 & $0.5 f_{x}$ \\
\hline OGLE-BLG-RRLYR-08148 & $0.28819496(4)$ & $0.177189(1)$ & 0.61482 & $0.1302(3)$ & 0.014 & \\
\hline OGLE-BLG-RRLYR-08158 & $0.32744431(4)$ & $0.2034111(10)$ & 0.62121 & $0.1285(3)$ & 0.012 & $0.5 f_{x}$ \\
\hline OGLE-BLG-RRLYR-08159 & $0.31507831(6)$ & $0.195915(1)$ & 0.6218 & $0.1229(4)$ & 0.02 & \\
\hline OGLE-BLG-RRLYR-08170 & $0.32305872(3)$ & $0.1994548(9)$ & 0.61739 & $0.1164(2)$ & 0.014 & \\
\hline OGLE-BLG-RRLYR-08177 & $0.28513618(4)$ & $0.1751747(9)$ & 0.61435 & $0.1380(3)$ & 0.014 & $\mathrm{bl}$ \\
\hline OGLE-BLG-RRLYR-08183 & $0.29385880(4)$ & $0.1805521(8)$ & 0.61442 & $0.1373(4)$ & 0.02 & \\
\hline OGLE-BLG-RRLYR-08186 & $0.29419968(3)$ & $0.1858289(9)$ & 0.63164 & $0.1160(2)$ & 0.012 & $0.5 f_{x}$ \\
\hline OGLE-BLG-RRLYR-08187 & $0.32335404(9)$ & $0.198424(2)$ & 0.61364 & $0.1272(6)$ & 0.016 & cand. \\
\hline \multirow[t]{2}{*}{ OGLE-BLG-RRLYR-08209 } & $0.25811797(3)$ & $0.1580910(7)$ & 0.61248 & $0.0962(2)$ & 0.02 & \\
\hline & $0.25811797(3)$ & $0.1605216(7)$ & 0.62189 & $0.0962(2)$ & 0.017 & \\
\hline \multirow{2}{*}{ OGLE-BLG-RRLYR-08214 } & $0.31067628(3)$ & $0.1905933(4)$ & 0.61348 & $0.1243(2)$ & 0.025 & $0.5 f_{x}$ \\
\hline & $0.31067628(3)$ & $0.1932697(9)$ & 0.62209 & $0.1243(2)$ & 0.014 & \\
\hline OGLE-BLG-RRLYR-08237 & $0.31762956(4)$ & $0.1946851(9)$ & 0.61293 & $0.1176(2)$ & 0.015 & \\
\hline OGLE-BLG-RRLYR-08248 & $0.28522012(3)$ & $0.1747361(8)$ & 0.61264 & $0.1338(2)$ & 0.013 & \\
\hline OGLE-BLG-RRLYR-08261 & $0.36067970(7)$ & $0.226948(2)$ & 0.62922 & $0.1141(3)$ & 0.013 & cand. \\
\hline \multirow[t]{2}{*}{ OGLE-BLG-RRLYR-08302 } & $0.29945276(3)$ & $0.1839611(6)$ & 0.61432 & $0.1309(2)$ & 0.018 & \\
\hline & $0.29945276(3)$ & $0.1857436(9)$ & 0.62028 & $0.1309(2)$ & 0.011 & \\
\hline \multirow[t]{2}{*}{ OGLE-BLG-RRLYR-08318 } & $0.30413328(3)$ & $0.1866294(10)$ & 0.61364 & $0.1347(2)$ & 0.011 & \\
\hline & $0.30413328(3)$ & $0.191738(1)$ & 0.63044 & $0.1347(2)$ & 0.008 & \\
\hline OGLE-BLG-RRLYR-08321 & $0.33110180(7)$ & $0.20322(2)$ & 0.61377 & $0.1356(5)$ & 0.021 & \\
\hline OGLE-BLG-RRLYR-08349 & $0.29708835(3)$ & $0.182476(2)$ & 0.61421 & $0.1297(2)$ & 0.023 & \\
\hline OGLE-BLG-RRLYR-08361 & $0.30775625(8)$ & $0.1889834(8)$ & 0.61407 & $0.1242(6)$ & 0.02 & \\
\hline OGLE-BLG-RRLYR-08387 & $0.30162029(4)$ & $0.1848786(5)$ & 0.61295 & $0.1149(3)$ & 0.03 & \\
\hline OGLE-BLG-RRLYR-08390 & $0.29068697(3)$ & $0.1776034(7)$ & 0.61098 & $0.1315(2)$ & 0.014 & \\
\hline OGLE-BLG-RRLYR-08395 & $0.27681496(3)$ & $0.1689393(7)$ & 0.6103 & $0.0928(2)$ & 0.022 & \\
\hline OGLE-BLG-RRLYR-08396 & $0.29395271(4)$ & $0.1804451(10)$ & 0.61386 & $0.1337(3)$ & 0.016 & \\
\hline OGLE-BLG-RRLYR-08433 & $0.31841475(7)$ & $0.19499(2)$ & 0.61238 & $0.1356(5)$ & 0.01 & \\
\hline OGLE-BLG-RRLYR-08435 & $0.28638926(2)$ & $0.1758577(8)$ & 0.61405 & $0.1434(2)$ & 0.017 & \\
\hline OGLE-BLG-RRLYR-08443 & $0.30693444(3)$ & $0.188158(1)$ & 0.61302 & $0.1558(4)$ & 0.012 & cand. \\
\hline OGLE-BLG-RRLYR-08453 & $0.30799097(9)$ & $0.188929(1)$ & 0.61342 & $0.1255(5)$ & 0.019 & cand. \\
\hline OGLE-BLG-RRLYR-08460 & $0.36490289(3)$ & $0.228340(1)$ & 0.62576 & $0.1132(1)$ & 0.01 & $0.5 f_{x}$ \\
\hline
\end{tabular}


Table A1 - Continued from previous page

\begin{tabular}{|c|c|c|c|c|c|c|}
\hline ID & $P_{1 \mathrm{O}}[\mathrm{d}]$ & $P_{\mathrm{X}}[\mathrm{d}]$ & $P_{1 \mathrm{O}} / P_{\mathrm{X}}$ & $A_{10}[\mathrm{mag}]$ & $A_{\mathrm{X}} / A_{1 \mathrm{O}}$ & Remarks \\
\hline OGLE-BLG-RRLYR-08463 & $0.34095394(4)$ & $0.2137211(2)$ & 0.62683 & $0.1402(2)$ & 0.069 & \\
\hline \multirow{2}{*}{ OGLE-BLG-RRLYR-08470 } & $0.35559718(5)$ & $0.224513(1)$ & 0.63137 & $0.1298(3)$ & 0.009 & cand. \\
\hline & $0.35559718(5)$ & $0.223686(2)$ & 0.62904 & $0.1298(3)$ & 0.009 & \\
\hline \multirow[t]{2}{*}{ OGLE-BLG-RRLYR-08475 } & $0.31094850(5)$ & $0.1904943(6)$ & 0.61262 & $0.1073(2)$ & 0.018 & \\
\hline & $0.31094850(5)$ & $0.1962916(7)$ & 0.63127 & $0.1073(2)$ & 0.014 & \\
\hline OGLE-BLG-RRLYR-08521 & $0.29705730(7)$ & $0.181918(1)$ & 0.6124 & $0.1160(5)$ & 0.017 & \\
\hline OGLE-BLG-RRLYR-08584 & $0.31624769(6)$ & $0.193909(1)$ & 0.61316 & $0.1114(3)$ & 0.019 & \\
\hline OGLE-BLG-RRLYR-08590 & $0.24166428(3)$ & $0.1484770(5)$ & 0.61439 & $0.0827(2)$ & 0.024 & \\
\hline OGLE-BLG-RRLYR-08591 & $0.30219949(3)$ & $0.1855363(6)$ & 0.61395 & $0.1245(2)$ & 0.028 & \\
\hline OGLE-BLG-RRLYR-08594 & $0.29978662(2)$ & $0.1835684(4)$ & 0.61233 & $0.1310(2)$ & 0.034 & \\
\hline OGLE-BLG-RRLYR-08597 & $0.32092790(4)$ & $0.2022931(10)$ & 0.63034 & $0.1367(3)$ & 0.015 & \\
\hline OGLE-BLG-RRLYR-08623 & $0.30822782(9)$ & $0.188471(1)$ & 0.61147 & $0.1166(6)$ & 0.028 & \\
\hline OGLE-BLG-RRLYR-08637 & $0.29545625(7)$ & $0.181557(1)$ & 0.6145 & $0.1361(6)$ & 0.019 & \\
\hline OGLE-BLG-RRLYR-08640 & $0.28459786(2)$ & $0.1746397(7)$ & 0.61364 & $0.1283(2)$ & 0.023 & $\mathrm{bl}$ \\
\hline OGLE-BLG-RRLYR-08653 & $0.36015945(9)$ & $0.2223243(10)$ & 0.61729 & $0.1271(4)$ & 0.008 & \\
\hline \multirow[t]{2}{*}{ OGLE-BLG-RRLYR-08662 } & $0.32229264(3)$ & $0.2034956(3)$ & 0.6314 & $0.1454(2)$ & 0.012 & $0.5 f_{x}$ \\
\hline & $0.32229264(3)$ & $0.2029785(6)$ & 0.6298 & $0.1454(2)$ & 0.01 & \\
\hline OGLE-BLG-RRLYR-08671 & $0.31310944(6)$ & $0.191805(1)$ & 0.61258 & $0.1239(4)$ & 0.016 & \\
\hline OGLE-BLG-RRLYR-08674 & $0.31692033(2)$ & $0.1941955(8)$ & 0.61276 & $0.1226(1)$ & 0.015 & $0.5 f_{x}$ \\
\hline OGLE-BLG-RRLYR-08691 & $0.31687267(7)$ & $0.194344(1)$ & 0.61332 & $0.1217(4)$ & 0.016 & cand. \\
\hline OGLE-BLG-RRLYR-08692 & $0.27143652(7)$ & $0.1660583(10)$ & 0.61178 & $0.0948(5)$ & 0.022 & cand. \\
\hline OGLE-BLG-RRLYR-08698 & $0.25439361(5)$ & $0.1558065(8)$ & 0.61246 & $0.0966(4)$ & 0.026 & \\
\hline OGLE-BLG-RRLYR-08709 & $0.31485250(6)$ & $0.193090(2)$ & 0.61327 & $0.1278(4)$ & 0.016 & \\
\hline OGLE-BLG-RRLYR-08714 & $0.34099890(2)$ & $0.209409(1)$ & 0.6141 & $0.1314(1)$ & 0.008 & \\
\hline \multirow[t]{2}{*}{ OGLE-BLG-RRLYR-08715 } & $0.36985794(3)$ & $0.233237(2)$ & 0.63061 & $0.1301(2)$ & 0.008 & $0.5 f_{x}$ \\
\hline & $0.36985794(3)$ & $0.231580(1)$ & 0.62613 & $0.1301(2)$ & 0.009 & \\
\hline OGLE-BLG-RRLYR-08716 & $0.28834207(3)$ & $0.1772761(7)$ & 0.61481 & $0.1353(3)$ & 0.018 & \\
\hline OGLE-BLG-RRLYR-08721 & $0.24131811(3)$ & $0.1475510(4)$ & 0.61144 & $0.0803(2)$ & 0.031 & \\
\hline \multirow[t]{2}{*}{ OGLE-BLG-RRLYR-08734 } & $0.30813817(3)$ & $0.1890078(6)$ & 0.61339 & $0.1280(2)$ & 0.019 & \\
\hline & $0.30813817(3)$ & $0.194526(1)$ & 0.63129 & $0.1280(2)$ & 0.009 & \\
\hline OGLE-BLG-RRLYR-08745 & $0.29344543(4)$ & $0.180267(1)$ & 0.61431 & $0.1298(3)$ & 0.015 & \\
\hline OGLE-BLG-RRLYR-08759 & $0.29707660(5)$ & $0.182046(1)$ & 0.61279 & $0.1301(4)$ & 0.017 & \\
\hline OGLE-BLG-RRLYR-08765 & $0.28219917(2)$ & $0.173344(1)$ & 0.61426 & $0.1451(2)$ & 0.014 & \\
\hline OGLE-BLG-RRLYR-08772 & $0.32408801(3)$ & $0.195900(1)$ & 0.60447 & $0.1369(2)$ & 0.008 & \\
\hline OGLE-BLG-RRLYR-08788 & $0.28739179(5)$ & $0.1763023(8)$ & 0.61346 & $0.1366(4)$ & 0.022 & \\
\hline \multirow[t]{2}{*}{ OGLE-BLG-RRLYR-08799 } & $0.31734930(3)$ & $0.194598(2)$ & 0.6132 & $0.1252(2)$ & 0.016 & $0.5 f_{x}$ \\
\hline & $0.31734930(3)$ & $0.2002600(4)$ & 0.63104 & $0.1252(2)$ & 0.01 & \\
\hline \multirow[t]{2}{*}{ OGLE-BLG-RRLYR-08806 } & $0.35863521(4)$ & $0.226553(1)$ & 0.63171 & $0.1228(2)$ & 0.009 & $0.5 f_{x}$ \\
\hline & $0.35863521(4)$ & $0.224304(2)$ & 0.62544 & $0.1228(2)$ & 0.007 & \\
\hline \multirow[t]{2}{*}{ OGLE-BLG-RRLYR-08818 } & $0.31261154(3)$ & $0.1919419(8)$ & 0.61399 & $0.1238(2)$ & 0.016 & $0.5 f_{x}$ \\
\hline & $0.31261154(3)$ & $0.194588(1)$ & 0.62246 & $0.1238(2)$ & 0.009 & \\
\hline OGLE-BLG-RRLYR-08824 & $0.30035643(2)$ & $0.1836967(3)$ & 0.6116 & $0.1230(2)$ & 0.03 & \\
\hline \multirow[t]{3}{*}{ OGLE-BLG-RRLYR-08826 } & $0.31251553(3)$ & $0.1972236(7)$ & 0.63108 & $0.1012(1)$ & 0.011 & $0.5 f_{x}$ \\
\hline & $0.31251553(3)$ & $0.1912645(7)$ & 0.61202 & $0.1012(1)$ & 0.011 & \\
\hline & $0.31251553(3)$ & $0.1972236(7)$ & 0.63108 & $0.1012(1)$ & 0.011 & \\
\hline \multirow[t]{2}{*}{ OGLE-BLG-RRLYR-08836 } & $0.31737227(4)$ & $0.1972519(9)$ & 0.62152 & $0.1224(2)$ & 0.016 & $0.5 f_{x}$ \\
\hline & $0.31737227(4)$ & $0.2002220(10)$ & 0.63087 & $0.1224(2)$ & 0.014 & \\
\hline OGLE-BLG-RRLYR-08844 & $0.28957197(2)$ & $0.1775254(3)$ & 0.61306 & $0.1289(2)$ & 0.022 & \\
\hline OGLE-BLG-RRLYR-08846 & $0.32865557(8)$ & $0.201662(1)$ & 0.6136 & $0.1192(4)$ & 0.017 & \\
\hline OGLE-BLG-RRLYR-08847 & $0.26403220(3)$ & $0.1618406(8)$ & 0.61296 & $0.0723(1)$ & 0.012 & \\
\hline OGLE-BLG-RRLYR-08863 & $0.28168676(5)$ & $0.1729094(4)$ & 0.61384 & $0.1388(5)$ & 0.01 & \\
\hline OGLE-BLG-RRLYR-08864 & $0.31455752(6)$ & $0.193435(8)$ & 0.61494 & $0.1467(4)$ & 0.014 & \\
\hline OGLE-BLG-RRLYR-08866 & $0.24141785(2)$ & $0.1480085(6)$ & 0.61308 & $0.1351(2)$ & 0.013 & \\
\hline OGLE-BLG-RRLYR- 08877 & $0.29017300(4)$ & $0.178173(1)$ & 0.61402 & $0.1284(5)$ & 0.022 & \\
\hline OGLE-BLG-RRLYR-08896 & $0.28189040(2)$ & $0.1737032(8)$ & 0.61621 & $0.1468(2)$ & 0.01 & \\
\hline OGLE-BLG-RRLYR-08898 & $0.27934085(2)$ & $0.1718724(7)$ & 0.61528 & $0.1524(2)$ & 0.01 & \\
\hline OGLE-BLG-RRLYR-08918 & $0.31323065(4)$ & $0.197364(1)$ & 0.63009 & $0.1339(3)$ & 0.01 & \\
\hline \multirow[t]{2}{*}{ OGLE-BLG-RRLYR-08920 } & $0.31500873(6)$ & $0.1931971(8)$ & 0.61331 & $0.1177(3)$ & 0.023 & \\
\hline & $0.31500873(6)$ & $0.198862(1)$ & 0.63129 & $0.1177(3)$ & 0.02 & \\
\hline
\end{tabular}


Table A1 - Continued from previous page

\begin{tabular}{c} 
ID \\
\hline OGLE-BLG-RRLYR-08945 \\
OGLE-BLG-RRLYR-08958 \\
OGLE-BLG-RRLYR-08968 \\
OGLE-BLG-RRLYR-08973 \\
OGLE-BLG-RRLYR-08980 \\
\\
OGLE-BLG-RRLYR-09009 \\
OGLE-BLG-RRLYR-09017 \\
OGLE-BLG-RRLYR-09030 \\
OGLE-BLG-RRLYR-09041 \\
OGLE-BLG-RRLYR-09056 \\
OGLE-BLG-RRLYR-09126 \\
OGLE-BLG-RRLYR-09134 \\
OGLE-BLG-RRLYR-09153 \\
OGLE-BLG-RRLYR-09163 \\
OGLE-BLG-RRLYR-09164 \\
OGLE-BLG-RRLYR-09201 \\
OGLE-BLG-RRLYR-09205
\end{tabular}

OGLE-BLG-RRLYR-09206 OGLE-BLG-RRLYR-09212 OGLE-BLG-RRLYR-09216 OGLE-BLG-RRLYR-09249 OGLE-BLG-RRLYR-09258 OGLE-BLG-RRLYR-09264 OGLE-BLG-RRLYR-09267 OGLE-BLG-RRLYR-09278 OGLE-BLG-RRLYR-09292 OGLE-BLG-RRLYR-09298 OGLE-BLG-RRLYR-09300 OGLE-BLG-RRLYR-09305

OGLE-BLG-RRLYR-09306 OGLE-BLG-RRLYR-09341 OGLE-BLG-RRLYR-09375 OGLE-BLG-RRLYR-09387 OGLE-BLG-RRLYR-09433 OGLE-BLG-RRLYR-09436 OGLE-BLG-RRLYR-09444

OGLE-BLG-RRLYR-09454 OGLE-BLG-RRLYR-09481

OGLE-BLG-RRLYR-09497 OGLE-BLG-RRLYR-09510 OGLE-BLG-RRLYR-09511 OGLE-BLG-RRLYR-09520 OGLE-BLG-RRLYR-09521

OGLE-BLG-RRLYR-09529 OGLE-BLG-RRLYR-09578

OGLE-BLG-RRLYR-09594

$P_{1 \mathrm{O}}[\mathrm{d}]$
$0.28842634(2)$

$0.29293145(2)$

$0.29176953(4)$

$0.30873469(4)$

$0.31356147(3)$

$0.31356147(3)$

$0.31356147(3)$

$0.365666(9)$

$0.27365999(3)$

$0.3234944(2)$

$0.32006284(4)$

$0.29471307(6)$

$0.32398909(3)$

$0.32398909(3)$

$0.29693279(3)$

$0.28929274(4)$

$0.29551923(4)$

$0.28639120(2)$

$0.30160851(3)$

$0.32121335(6)$

$0.32121335(6)$

$0.32121335(6)$

$0.29500222(4)$

$0.234955620(10)$

$0.31039506(7)$

$0.30475025(3)$

$0.27766821(4)$

$0.30322692(4)$

$0.24987659(3)$

$0.27352204(4)$

$0.30442505(4)$

$0.31963205(3)$

$0.32739840(5)$

$0.30294829(2)$

$0.30294829(2)$

$0.29495396(5)$

$0.31542622(3)$

$0.28790934(4)$

$0.27857884(2)$

$0.27294961(5)$

$0.33011156(6)$

$0.32376783(4)$

$0.32376783(4)$

$0.32376783(4)$

$0.282806390(10)$

$0.31772067(5)$

$0.31772067(5)$

$0.29119414(3)$

$0.31504286(5)$

$0.30282659(3)$

$0.24892788(2)$

$0.32317475(3)$

$0.32317475(3)$

$0.32317475(3)$

$0.30692883(2)$

$0.30527835(3)$

$0.30527835(3)$

$0.32046610(4)$

$0.32046610(4)$
$P_{\mathrm{X}}[\mathrm{d}]$

$0.1767927(4)$

$0.179165(2)$

$0.178368(1)$

$0.1893992(5)$

$0.1916661(9)$

$0.1950056(10)$

$0.197879(1)$

$0.230207(1)$

$0.1678242(8)$

$0.198395(1)$

$0.1967554(7)$

$0.180906(1)$

$0.2013770(7)$

$0.1984768(9)$

$0.1824143(7)$

$0.177752(1)$

$0.1810592(9)$

$0.1755787(4)$

$0.1841458(5)$

$0.199666(1)$

$0.196762(2)$

$0.202629(1)$

$0.1807227(7)$

$0.1443403(5)$

$0.1904603(9)$

$0.1878680(2)$

$0.175151(1)$

$0.1879673(10)$

$0.1530163(5)$

$0.1678805(7)$

$0.185398(1)$

$0.1965480(5)$

$0.206285(1)$

$0.1859437(3)$

$0.1885224(9)$

$0.181032(1)$

$0.1922456(9)$

$0.1764221(9)$

$0.1710075(7)$

$0.1645013(7)$

$0.2022466(8)$

$0.204370(1)$

$0.198608(1)$

$0.204370(1)$

$0.1733403(4)$

$0.1950060(10)$

$0.197611(1)$

$0.178485(1)$

$0.195750(1)$

$0.1897132(8)$

$0.1528190(7)$

$0.198180(1)$

0.200907(1)

$0.203790(1)$

$0.18709572(8)$

$0.1865925(9)$

$0.192381(1)$

$0.2022054(8)$ $0.199301(1)$

$P_{1 \mathrm{O}} / P_{\mathrm{X}}$

$A_{10}[\mathrm{mag}]$ $A_{\mathrm{X}} / A_{1 \mathrm{O}} \quad$ Remarks

0.018

$\begin{array}{lll}0.61163 & 0.1534(3) & 0.012\end{array}$

0.61133

$0.1272(3)$

0.012

$\begin{array}{lll}0.61347 & 0.1272(3) & 0.021\end{array}$

$\begin{array}{lll}0.61126 & 0.1286(2) & 0.009\end{array}$

$\begin{array}{lll}0.62191 & 0.1286(2) & 0.009\end{array}$

$\begin{array}{lll}0.63107 & 0.1286(2) & 0.008\end{array}$

$\begin{array}{lll}0.62956 & 0.12(1) & 0.011\end{array}$

$\begin{array}{lll}0.61326 & 0.1383(3) & 0.016\end{array}$

$\begin{array}{lll}0.61329 & 0.1242(9) & 0.018\end{array}$

$\begin{array}{lll}0.61474 & 0.1461(3) & 0.022\end{array}$

$\begin{array}{lll}0.61384 & 0.1331(5) & 0.024\end{array}$

$\begin{array}{lll}0.62155 & 0.1240(2) & 0.017\end{array}$

$\begin{array}{lll}0.6126 & 0.1240(2) & 0.011\end{array}$

$\begin{array}{lll}0.61433 & 0.1293(3) & 0.024\end{array}$

$\begin{array}{lll}0.61444 & 0.1228(3) & 0.013\end{array}$

$\begin{array}{lll}0.61268 & 0.1160(3) & 0.014\end{array}$

$\begin{array}{lll}0.61307 & 0.1435(2) & 0.015\end{array}$

$\begin{array}{lll}0.61055 & 0.1324(3) & 0.024\end{array}$

$\begin{array}{lll}0.6216 & 0.1193(4) & 0.02\end{array}$

$\begin{array}{lll}0.61256 & 0.1193(4) & 0.015\end{array}$

$\begin{array}{lll}0.63082 & 0.1193(4) & 0.018\end{array}$

$\begin{array}{lll}0.61261 & 0.1216(3) & 0.021\end{array}$

$\begin{array}{lll}0.61433 & 0.1246(2) & 0.012\end{array}$

$\begin{array}{lll}0.61361 & 0.1330(4) & 0.023\end{array}$

$\begin{array}{lll}0.61647 & 0.1133(2) & 0.013\end{array}$

$\begin{array}{lll}0.63079 & 0.1013(2) & 0.014\end{array}$

$\begin{array}{lll}0.61989 & 0.1288(3) & 0.012\end{array}$

$\begin{array}{lll}0.61237 & 0.1217(3) & 0.021\end{array}$

$\begin{array}{lll}0.61377 & 0.1173(4) & 0.026\end{array}$

$\begin{array}{lll}0.60901 & 0.1401(3) & 0.017\end{array}$

$\begin{array}{lll}0.61492 & 0.1082(2) & 0.013\end{array}$

$\begin{array}{lll}0.63007 & 0.0484(1) & 0.021\end{array}$

$\begin{array}{lll}0.61378 & 0.1276(1) & 0.024\end{array}$

$\begin{array}{lll}0.62229 & 0.1276(1) & 0.008\end{array}$

$\begin{array}{lll}0.61376 & 0.1378(4) & 0.01\end{array}$

$\begin{array}{lll}0.60948 & 0.1252(2) & 0.01\end{array}$

$\begin{array}{lll}0.61277 & 0.1428(4) & 0.018\end{array}$

$\begin{array}{lll}0.61386 & 0.1484(3) & 0.013\end{array}$

$\begin{array}{lll}0.60268 & 0.1227(4) & 0.016\end{array}$

$\begin{array}{lll}0.61266 & 0.1216(3) & 0.011\end{array}$

$\begin{array}{lll}0.63122 & 0.1250(3) & 0.015\end{array}$

$\begin{array}{lll}0.61343 & 0.1250(3) & 0.014\end{array}$

$\begin{array}{lll}0.63122 & 0.1250(3) & 0.015\end{array}$

$\begin{array}{lll}0.61293 & 0.1439(2) & 0.018\end{array}$

$\begin{array}{lll}0.61377 & 0.1237(4) & 0.019\end{array}$

$\begin{array}{lll}0.62196 & 0.1237(4) & 0.015\end{array}$

$\begin{array}{lll}0.61294 & 0.1420(3) & 0.011\end{array}$

$\begin{array}{lll}0.62134 & 0.1162(3) & 0.015\end{array}$

$\begin{array}{lll}0.62647 & 0.1099(2) & 0.01\end{array}$

$\begin{array}{lll}0.61391 & 0.1091(2) & 0.013\end{array}$

$\begin{array}{lll}0.61323 & 0.1147(2) & 0.008\end{array}$

$\begin{array}{lll}0.62167 & 0.1147(2) & 0.008\end{array}$

$\begin{array}{lll}0.63059 & 0.1147(2) & 0.007\end{array}$

$\begin{array}{lll}0.60957 & 0.1254(1) & 0.024\end{array}$

$\begin{array}{lll}0.61122 & 0.1342(2) & 0.013\end{array}$

$\begin{array}{lll}0.63018 & 0.1342(2) & 0.01\end{array}$

$\begin{array}{llll}0.63097 & 0.1222(2) & 0.017 & 0.5 f_{x}\end{array}$ $0.62191 \quad 0.1222(2)$ bl

cand.

cand.

$0.5 f_{x}$

RRd

cand.

$0.5 f_{x}$

$0.5 f_{x}$, cand.

cand.

$0.5 f_{x}$

$0.5 f_{x}$

cand.

$0.5 f_{x}$ 0.013 
Table A1 - Continued from previous page

\begin{tabular}{|c|c|c|c|c|c|c|}
\hline ID & $P_{1 \mathrm{O}}[\mathrm{d}]$ & $P_{\mathrm{X}}[\mathrm{d}]$ & $P_{1 \mathrm{O}} / P_{\mathrm{X}}$ & $A_{10}[\mathrm{mag}]$ & $A_{\mathrm{X}} / A_{1 \mathrm{O}}$ & Remarks \\
\hline OGLE-BLG-RRLYR-09598 & $0.32928412(5)$ & $0.207587(2)$ & 0.63042 & $0.1077(2)$ & 0.009 & \\
\hline OGLE-BLG-RRLYR-09616 & $0.3428505(3)$ & $0.215927(2)$ & 0.6298 & $0.093(1)$ & 0.075 & cand. \\
\hline OGLE-BLG-RRLYR-09620 & $0.29163358(5)$ & $0.1788822(5)$ & 0.61338 & $0.1320(4)$ & 0.036 & \\
\hline OGLE-BLG-RRLYR-09631 & $0.280272690(10)$ & $0.1723691(6)$ & 0.615 & $0.1464(1)$ & 0.009 & \\
\hline OGLE-BLG-RRLYR-09637 & $0.29648222(4)$ & $0.181560(1)$ & 0.61238 & $0.1398(4)$ & 0.014 & \\
\hline \multirow[t]{2}{*}{ OGLE-BLG-RRLYR-09647 } & $0.26891630(4)$ & $0.1703260(8)$ & 0.63338 & $0.0869(3)$ & 0.02 & $0.5 f_{x}$ \\
\hline & $0.26891630(4)$ & $0.1690018(9)$ & 0.62846 & $0.0869(3)$ & 0.016 & \\
\hline OGLE-BLG-RRLYR-09649 & $0.30892587(5)$ & $0.1898070(8)$ & 0.61441 & $0.1160(3)$ & 0.025 & \\
\hline OGLE-BLG-RRLYR-09656 & $0.42506764(7)$ & $0.265487(1)$ & 0.62458 & $0.0998(2)$ & 0.018 & cand. \\
\hline OGLE-BLG-RRLYR-09665 & $0.29925226(6)$ & $0.1835184(9)$ & 0.61326 & $0.1174(4)$ & 0.028 & \\
\hline OGLE-BLG-RRLYR-09696 & $0.308380360(10)$ & $0.1877204(9)$ & 0.60873 & $0.1107(1)$ & 0.004 & \\
\hline OGLE-BLG-RRLYR-09703 & $0.29850683(5)$ & $0.182792(2)$ & 0.61235 & $0.1193(3)$ & 0.017 & \\
\hline \multirow[t]{2}{*}{ OGLE-BLG-RRLYR-09733 } & $0.31378022(3)$ & $0.1921045(2)$ & 0.61223 & $0.1213(2)$ & 0.013 & $0.5 f_{x}$ \\
\hline & $0.31378022(3)$ & $0.197952(2)$ & 0.63086 & $0.1213(2)$ & 0.012 & \\
\hline OGLE-BLG-RRLYR-09749 & $0.32134035(4)$ & $0.196237(1)$ & 0.61068 & $0.1239(2)$ & 0.01 & \\
\hline OGLE-BLG-RRLYR-09771 & $0.26098423(3)$ & $0.1599148(7)$ & 0.61274 & $0.1232(3)$ & 0.016 & \\
\hline OGLE-BLG-RRLYR-09787 & $0.35051026(6)$ & $0.219962(2)$ & 0.62755 & $0.1066(3)$ & 0.014 & \\
\hline OGLE-BLG-RRLYR-09795 & $0.31619057(2)$ & $0.1943701(9)$ & 0.61472 & $0.1584(2)$ & 0.006 & \\
\hline OGLE-BLG-RRLYR-09809 & $0.29856692(3)$ & $0.1830241(7)$ & 0.61301 & $0.1461(3)$ & 0.011 & \\
\hline \multirow[t]{2}{*}{ OGLE-BLG-RRLYR-09811 } & $0.31326458(3)$ & $0.1920059(4)$ & 0.61292 & $0.1237(2)$ & 0.016 & $\mathrm{bl}$ \\
\hline & $0.31326458(3)$ & $0.1947839(6)$ & 0.62179 & $0.1237(2)$ & 0.012 & \\
\hline OGLE-BLG-RRLYR-09813 & $0.30360388(3)$ & $0.186154(1)$ & 0.61315 & $0.1265(2)$ & 0.013 & \\
\hline OGLE-BLG-RRLYR-09825 & $0.34397868(3)$ & $0.2116645(5)$ & 0.61534 & $0.1296(1)$ & 0.021 & \\
\hline OGLE-BLG-RRLYR-09868 & $0.29430171(3)$ & $0.1804498(4)$ & 0.61315 & $0.1362(2)$ & 0.023 & $\mathrm{bl}$ \\
\hline \multirow[t]{2}{*}{ OGLE-BLG-RRLYR-09891 } & $0.31104277(4)$ & $0.1905705(10)$ & 0.61268 & $0.1329(3)$ & 0.011 & \\
\hline & $0.31104277(4)$ & $0.194075(1)$ & 0.62395 & $0.1329(3)$ & 0.011 & \\
\hline OGLE-BLG-RRLYR-09892 & $0.35423124(7)$ & $0.216563(2)$ & 0.61136 & $0.1358(2)$ & 0.008 & \\
\hline OGLE-BLG-RRLYR-09920 & $0.32702140(7)$ & $0.200109(1)$ & 0.61191 & $0.1349(4)$ & 0.012 & cand. \\
\hline OGLE-BLG-RRLYR-09927 & $0.29450670(3)$ & $0.1809330(8)$ & 0.61436 & $0.1341(3)$ & 0.016 & \\
\hline OGLE-BLG-RRLYR-09929 & $0.29916617(2)$ & $0.1834687(5)$ & 0.61327 & $0.1261(2)$ & 0.02 & \\
\hline \multirow[t]{2}{*}{ OGLE-BLG-RRLYR-09933 } & $0.27949519(6)$ & $0.1735978(9)$ & 0.62111 & $0.1132(4)$ & 0.019 & \\
\hline & $0.27949519(6)$ & $0.1765930(8)$ & 0.63183 & $0.1132(4)$ & 0.016 & \\
\hline OGLE-BLG-RRLYR-09977 & $0.28851306(2)$ & $0.1773083(7)$ & 0.61456 & $0.1352(2)$ & 0.01 & \\
\hline \multirow[t]{2}{*}{ OGLE-BLG-RRLYR-09985 } & $0.31675326(4)$ & $0.1970058(8)$ & 0.62195 & $0.1297(3)$ & 0.016 & $0.5 f_{x}$ \\
\hline & $0.31675326(4)$ & $0.1942740(10)$ & 0.61333 & $0.1297(3)$ & 0.012 & \\
\hline OGLE-BLG-RRLYR-09987 & $0.28091096(2)$ & $0.1728011(8)$ & 0.61515 & $0.1489(2)$ & 0.009 & \\
\hline OGLE-BLG-RRLYR-09988 & $0.30615695(7)$ & $0.187003(1)$ & 0.61081 & $0.1276(5)$ & 0.02 & \\
\hline OGLE-BLG-RRLYR-10000 & $0.29739041(3)$ & $0.1820431(7)$ & 0.61214 & $0.1287(3)$ & 0.024 & \\
\hline OGLE-BLG-RRLYR-10008 & $0.30434827(5)$ & $0.1872793(6)$ & 0.61535 & $0.1281(4)$ & 0.022 & \\
\hline \multirow[t]{2}{*}{ OGLE-BLG-RRLYR-10037 } & $0.32979605(2)$ & $0.2077514(4)$ & 0.62994 & $0.1311(1)$ & 0.021 & $0.5 f_{x}$ \\
\hline & $0.32979605(2)$ & $0.2079973(7)$ & 0.63068 & $0.1311(1)$ & 0.014 & \\
\hline OGLE-BLG-RRLYR-10040 & $0.31351304(3)$ & $0.1922984(8)$ & 0.61337 & $0.1316(2)$ & 0.011 & \\
\hline OGLE-BLG-RRLYR-10056 & $0.29856514(5)$ & $0.1833661(7)$ & 0.61416 & $0.1256(3)$ & 0.03 & \\
\hline OGLE-BLG-RRLYR-10061 & $0.26253029(4)$ & $0.1602975(4)$ & 0.61059 & $0.0614(2)$ & 0.042 & \\
\hline OGLE-BLG-RRLYR-10076 & $0.32133078(6)$ & $0.1972129(8)$ & 0.61374 & $0.1274(4)$ & 0.024 & \\
\hline OGLE-BLG-RRLYR-10087 & $0.29580701(3)$ & $0.1812962(6)$ & 0.61289 & $0.1359(2)$ & 0.018 & \\
\hline OGLE-BLG-RRLYR-10116 & $0.27567307(3)$ & $0.169064(1)$ & 0.61328 & $0.1268(3)$ & 0.017 & \\
\hline \multirow[t]{3}{*}{ OGLE-BLG-RRLYR-10119 } & $0.31540045(3)$ & $0.199050(1)$ & 0.6311 & $0.1148(2)$ & 0.012 & \\
\hline & $0.31540045(3)$ & $0.193523(1)$ & 0.61358 & $0.1148(2)$ & 0.011 & \\
\hline & $0.31540045(3)$ & $0.196063(1)$ & 0.62163 & $0.1148(2)$ & 0.01 & \\
\hline OGLE-BLG-RRLYR-10121 & $0.3159374(2)$ & $0.193770(2)$ & 0.61332 & $0.1224(5)$ & 0.016 & \\
\hline OGLE-BLG-RRLYR-10126 & $0.25334599(9)$ & $0.155111(1)$ & 0.61225 & $0.0969(6)$ & 0.032 & cand. \\
\hline OGLE-BLG-RRLYR-10134 & $0.31201373(3)$ & $0.1925866(6)$ & 0.61724 & $0.1317(2)$ & 0.014 & \\
\hline OGLE-BLG-RRLYR-10145 & $0.3222939(2)$ & $0.197441(1)$ & 0.61261 & $0.1244(9)$ & 0.018 & \\
\hline \multirow[t]{2}{*}{ OGLE-BLG-RRLYR-10150 } & $0.32642369(4)$ & $0.205507(2)$ & 0.62957 & $0.1295(3)$ & 0.008 & $0.5 f_{x}$ \\
\hline & $0.32642369(4)$ & $0.205665(2)$ & 0.63006 & $0.1295(3)$ & 0.008 & \\
\hline \multirow[t]{2}{*}{ OGLE-BLG-RRLYR-10184 } & $0.31114642(4)$ & $0.1900107(7)$ & 0.61068 & $0.1298(3)$ & 0.016 & \\
\hline & $0.31114642(4)$ & $0.196075(1)$ & 0.63017 & $0.1298(3)$ & 0.01 & \\
\hline OGLE-BLG-RRLYR-10187 & $0.35272129(6)$ & $0.221273(1)$ & 0.62733 & $0.1154(3)$ & 0.016 & $0.5 f_{x}$ \\
\hline
\end{tabular}


Table A1 - Continued from previous page

\begin{tabular}{c} 
ID \\
\hline OGLE-BLG-RRLYR-10230 \\
OGLE-BLG-RRLYR-10244 \\
OGLE-BLG-RRLYR-10253 \\
OGLE-BLG-RRLYR-10257 \\
OGLE-BLG-RRLYR-10262 \\
\\
OGLE-BLG-RRLYR-10275 \\
OGLE-BLG-RRLYR-10289 \\
OGLE-BLG-RRLYR-10296 \\
OGLE-BLG-RRLYR-10308 \\
OGLE-BLG-RRLYR-10316 \\
OGLE-BLG-RRLYR-10320 \\
OGLE-BLG-RRLYR-10323 \\
OGLE-BLG-RRLYR-10338
\end{tabular}

OGLE-BLG-RRLYR-10350 OGLE-BLG-RRLYR-10352 OGLE-BLG-RRLYR-10361

OGLE-BLG-RRLYR-10366 OGLE-BLG-RRLYR-10369 OGLE-BLG-RRLYR-10371 OGLE-BLG-RRLYR-10378 OGLE-BLG-RRLYR-10380 OGLE-BLG-RRLYR-10396 OGLE-BLG-RRLYR-10398 OGLE-BLG-RRLYR-10403 OGLE-BLG-RRLYR-10413 OGLE-BLG-RRLYR-10425 OGLE-BLG-RRLYR-10426 OGLE-BLG-RRLYR-10445 OGLE-BLG-RRLYR-10447 OGLE-BLG-RRLYR-10458 OGLE-BLG-RRLYR-10467 OGLE-BLG-RRLYR-10511 OGLE-BLG-RRLYR-10534

OGLE-BLG-RRLYR-10541 OGLE-BLG-RRLYR-10543 OGLE-BLG-RRLYR-10549 OGLE-BLG-RRLYR-10554 OGLE-BLG-RRLYR-10565 OGLE-BLG-RRLYR-10566 OGLE-BLG-RRLYR-10587 OGLE-BLG-RRLYR-10597 OGLE-BLG-RRLYR-10614

OGLE-BLG-RRLYR-10627 OGLE-BLG-RRLYR-10643 OGLE-BLG-RRLYR-10653 OGLE-BLG-RRLYR-10663 OGLE-BLG-RRLYR-10665 OGLE-BLG-RRLYR-10675 OGLE-BLG-RRLYR-10685

OGLE-BLG-RRLYR-10724

\begin{tabular}{|c|c|c|c|c|c|}
\hline$P_{10}[\mathrm{~d}]$ & $P_{\mathrm{X}}[\mathrm{d}]$ & $P_{1 \mathrm{O}} / P_{\mathrm{X}}$ & $A_{10}[\mathrm{mag}]$ & $A_{\mathrm{X}} / A_{1 \mathrm{O}}$ & Remarks \\
\hline $0.31078131(4)$ & $0.1906684(2)$ & 0.61351 & $0.1270(3)$ & 0.029 & \\
\hline $0.31346263(3)$ & $0.1922358(5)$ & 0.61327 & $0.1237(2)$ & 0.023 & \\
\hline $0.29657997(2)$ & $0.1816522(6)$ & 0.61249 & $0.1554(2)$ & 0.014 & \\
\hline $0.31198004(3)$ & $0.1911386(7)$ & 0.61266 & $0.1265(2)$ & 0.017 & $0.5 f_{x}$ \\
\hline $0.316221310(10)$ & $0.199536(5)$ & 0.631 & $0.1129(2)$ & 0.018 & \\
\hline $0.316221310(10)$ & $0.19401(1)$ & 0.61353 & $0.1129(2)$ & 0.009 & \\
\hline $0.316221310(10)$ & $0.199536(5)$ & 0.631 & $0.1129(2)$ & 0.018 & \\
\hline $0.28162771(2)$ & $0.173175(1)$ & 0.61491 & $0.1365(2)$ & 0.012 & $\mathrm{bl}$ \\
\hline $0.30849841(8)$ & $0.188907(1)$ & 0.61234 & $0.1290(5)$ & 0.019 & \\
\hline $0.37242529(7)$ & $0.227177(2)$ & 0.60999 & $0.1187(3)$ & 0.008 & \\
\hline $0.30966512(4)$ & $0.190125(1)$ & 0.61397 & $0.1242(2)$ & 0.014 & \\
\hline $0.24231389(4)$ & $0.1485661(9)$ & 0.61311 & $0.1140(4)$ & 0.018 & \\
\hline $0.32335494(5)$ & 0.197938(1) & 0.61214 & $0.1186(3)$ & 0.017 & \\
\hline $0.31555429(3)$ & $0.1935619(8)$ & 0.6134 & $0.1221(2)$ & 0.014 & \\
\hline $0.33789403(3)$ & $0.2126097(10)$ & 0.62922 & $0.1285(2)$ & 0.011 & $0.5 f_{x}$ \\
\hline $0.33789403(3)$ & $0.2134688(9)$ & 0.63176 & $0.1285(2)$ & 0.011 & \\
\hline $0.30033053(5)$ & $0.1891142(10)$ & 0.62969 & $0.1107(3)$ & 0.014 & cand. \\
\hline $0.29856803(3)$ & $0.1834115(6)$ & 0.6143 & $0.1303(2)$ & 0.02 & \\
\hline $0.31361371(3)$ & $0.1920109(4)$ & 0.61225 & $0.1227(1)$ & 0.013 & \\
\hline $0.31361371(3)$ & $0.194743(1)$ & 0.62096 & $0.1227(1)$ & 0.01 & \\
\hline $0.29588318(5)$ & $0.1817827(8)$ & 0.61437 & $0.1288(4)$ & 0.023 & \\
\hline $0.32170650(5)$ & $0.203250(2)$ & 0.63179 & $0.1126(3)$ & 0.012 & RRd \\
\hline $0.289345890(10)$ & $0.177505(2)$ & 0.61347 & $0.1359(1)$ & 0.015 & \\
\hline $0.25471704(2)$ & $0.1563983(4)$ & 0.61401 & $0.0962(1)$ & 0.019 & \\
\hline $0.28001463(3)$ & $0.172271(1)$ & 0.61522 & $0.1489(3)$ & 0.011 & \\
\hline $0.32214186(3)$ & $0.1973254(9)$ & 0.61254 & $0.1180(2)$ & 0.01 & \\
\hline $0.30264669(3)$ & $0.1851273(8)$ & 0.61169 & $0.1258(2)$ & 0.023 & \\
\hline $0.30535610(3)$ & $0.1868309(9)$ & 0.61185 & $0.1567(3)$ & 0.011 & \\
\hline $0.34605753(4)$ & $0.211788(1)$ & 0.612 & $0.1375(2)$ & 0.008 & \\
\hline $0.3201621(2)$ & $0.1965502(7)$ & 0.61391 & $0.1299(3)$ & 0.024 & \\
\hline $0.30200465(4)$ & $0.1851452(10)$ & 0.61305 & $0.1276(3)$ & 0.016 & \\
\hline $0.30371431(6)$ & $0.186197(1)$ & 0.61307 & $0.1377(8)$ & 0.013 & \\
\hline $0.31187343(2)$ & $0.1909987(7)$ & 0.61242 & $0.1209(2)$ & 0.015 & \\
\hline $0.32412262(6)$ & $0.201561(2)$ & 0.62187 & $0.1223(4)$ & 0.013 & \\
\hline $0.28446855(7)$ & $0.1743336(5)$ & 0.61284 & $0.1389(2)$ & 0.019 & \\
\hline $0.37342673(7)$ & $0.233405(2)$ & 0.62504 & $0.1024(3)$ & 0.013 & \\
\hline $0.31529923(3)$ & $0.1934537(7)$ & 0.61356 & $0.1111(1)$ & 0.014 & $0.5 f_{x}$ \\
\hline $0.31529923(3)$ & $0.1959797(8)$ & 0.62157 & $0.1111(1)$ & 0.009 & \\
\hline $0.31529923(3)$ & $0.1991058(7)$ & 0.63148 & $0.1111(1)$ & 0.012 & \\
\hline $0.32542169(6)$ & $0.206105(2)$ & 0.63335 & $0.1301(4)$ & 0.014 & \\
\hline $0.34334765(2)$ & $0.2101539(7)$ & 0.61207 & $0.14430(10)$ & 0.008 & \\
\hline $0.28611102(3)$ & $0.1754882(4)$ & 0.61336 & $0.1372(3)$ & 0.026 & \\
\hline $0.2996659(2)$ & $0.1836414(8)$ & 0.61282 & $0.1117(8)$ & 0.013 & \\
\hline $0.2459469(1)$ & $0.1494820(7)$ & 0.60778 & $0.0926(8)$ & 0.021 & \\
\hline $0.31183172(8)$ & $0.191062(2)$ & 0.61271 & $0.1275(5)$ & 0.016 & \\
\hline $0.28296282(3)$ & $0.174229(1)$ & 0.61573 & $0.1470(3)$ & 0.01 & \\
\hline $0.29134346(5)$ & $0.1792114(9)$ & 0.61512 & $0.1416(5)$ & 0.02 & \\
\hline $0.31448656(4)$ & $0.1925720(9)$ & 0.61234 & $0.1167(2)$ & 0.014 & \\
\hline $0.31448656(4)$ & $0.195584(1)$ & 0.62192 & $0.1167(2)$ & 0.012 & \\
\hline $0.31448656(4)$ & $0.198513(1)$ & 0.63123 & $0.1167(2)$ & 0.01 & \\
\hline $0.29039790(5)$ & $0.178389(1)$ & 0.61429 & $0.1521(4)$ & 0.013 & \\
\hline $0.30572141(10)$ & $0.187242(1)$ & 0.61246 & $0.1190(6)$ & 0.014 & \\
\hline $0.3273004(2)$ & $0.201264(2)$ & 0.61492 & $0.107(1)$ & 0.032 & \\
\hline $0.276997300(10)$ & $0.1697823(7)$ & 0.61294 & $0.0859(3)$ & 0.024 & \\
\hline $0.31955224(7)$ & $0.201717(2)$ & 0.63125 & $0.1307(4)$ & 0.016 & \\
\hline $0.31939810(5)$ & $0.200920(1)$ & 0.62906 & $0.1263(7)$ & 0.025 & \\
\hline $0.30007336(3)$ & $0.1841198(5)$ & 0.61358 & $0.1316(2)$ & 0.02 & \\
\hline $0.30007336(3)$ & $0.1867025(10)$ & 0.62219 & $0.1316(2)$ & 0.009 & \\
\hline $0.28130622(2)$ & $0.1725182(10)$ & 0.61328 & $0.1428(6)$ & 0.017 & cand. \\
\hline
\end{tabular}

Continued on next page 
Table A1 - Continued from previous page

\begin{tabular}{c} 
ID \\
\hline OGLE-BLG-RRLYR-10733 \\
OGLE-BLG-RRLYR-10744 \\
OGLE-BLG-RRLYR-10747 \\
\\
OGLE-BLG-RRLYR-10756 \\
OGLE-BLG-RRLYR-10760 \\
OGLE-BLG-RRLYR-10771 \\
OGLE-BLG-RRLYR-10787 \\
OGLE-BLG-RRLYR-10789 \\
OGLE-BLG-RRLYR-10793 \\
OGLE-BLG-RRLYR-10795 \\
OGLE-BLG-RRLYR-10796
\end{tabular}

OGLE-BLG-RRLYR-10818 OGLE-BLG-RRLYR-10825

OGLE-BLG-RRLYR-10851 OGLE-BLG-RRLYR-10857 OGLE-BLG-RRLYR-10869 OGLE-BLG-RRLYR-10902 OGLE-BLG-RRLYR-10914 OGLE-BLG-RRLYR-10954 OGLE-BLG-RRLYR-10960 OGLE-BLG-RRLYR-10968

OGLE-BLG-RRLYR-10980 OGLE-BLG-RRLYR-10988 OGLE-BLG-RRLYR-11005 OGLE-BLG-RRLYR-11015 OGLE-BLG-RRLYR-11017 OGLE-BLG-RRLYR-11043 OGLE-BLG-RRLYR-11062 OGLE-BLG-RRLYR-11063 OGLE-BLG-RRLYR-11068 OGLE-BLG-RRLYR-11072

OGLE-BLG-RRLYR-11097 OGLE-BLG-RRLYR-11107

OGLE-BLG-RRLYR-11109 OGLE-BLG-RRLYR-11112 OGLE-BLG-RRLYR-11119 OGLE-BLG-RRLYR-11126 OGLE-BLG-RRLYR-11136

OGLE-BLG-RRLYR-11151 OGLE-BLG-RRLYR-11153 OGLE-BLG-RRLYR-11162

OGLE-BLG-RRLYR-11186 OGLE-BLG-RRLYR-11193 OGLE-BLG-RRLYR-11197 OGLE-BLG-RRLYR-11220 OGLE-BLG-RRLYR-11225 OGLE-BLG-RRLYR-11234 OGLE-BLG-RRLYR-11239

$P_{1 \mathrm{O}}[\mathrm{d}]$
$0.29313633(6)$

$0.35576412(8)$

$0.31793074(5)$

$0.31793074(5)$

$0.31793074(5)$

$0.2965903(2)$

$0.30134275(5)$

$0.31628683(5)$

$0.27650842(4)$

$0.31145344(7)$

$0.28357416(4)$

$0.30200831(4)$

$0.31909935(4)$

$0.31909935(4)$

$0.31909935(4)$

$0.26327469(4)$

$0.31695649(3)$

$0.31695649(3)$

$0.29511973(7)$

$0.24375427(2)$

$0.30187980(5)$

$0.33176630(3)$

$0.33143607(3)$

$0.23666005(4)$

$0.27924643(5)$

$0.32060825(7)$

$0.32060825(7)$

$0.29690954(8)$

$0.28179202(2)$

$0.28140860(3)$

$0.28957510(2)$

$0.26183405(3)$

$0.30032723(7)$

$0.3375229(1)$

$0.28947151(5)$

$0.29720790(3)$

$0.31601750(4)$

$0.31601750(4)$

$0.30815806(7)$

$0.30865448(2)$

$0.30865448(2)$

$0.31716288(6)$

$0.33175364(4)$

$0.27956130(2)$

$0.30215331(6)$

$0.31712352(3)$

$0.31712352(3)$

$0.31712352(3)$

$0.29670197(4)$

$0.28868401(6)$

$0.29134904(6)$

$0.29134904(6)$

$0.30101587(6)$

$0.36260234(2)$

$0.32276003(4)$

$0.27469620(4)$

$0.30255149(4)$

$0.35300247(8)$ $0.32628659(4)$
$P_{\mathrm{X}}[\mathrm{d}]$

$0.1801633(5)$

$0.218500(2)$

$0.1976417(3)$

$0.1947846(6)$

$0.2005261(9)$

$0.182217(1)$

$0.184586(1)$

$0.193939(1)$

$0.1696608(8)$

$0.190693(10)$

$0.1738193(10)$

$0.1853230(5)$

$0.1954256(8)$

$0.198505(1)$

$0.201310(1)$

$0.1612516(6)$

$0.1942610(8)$

$0.1970008(8)$

$0.180820(1)$

$0.1500403(7)$

$0.184652(1)$

$0.209522(1)$

$0.202229(1)$

$0.144831(2)$

$0.1711860(9)$

$0.199254(1)$

$0.196359(2)$

$0.182435(1)$

$0.1732004(9)$

$0.1728788(7)$

$0.1776282(8)$

$0.1603990(7)$

$0.183046(1)$

$0.212858(2)$

$0.1778433(9)$

$0.1822595(7)$

0.199391(1)

$0.193847(2)$

$0.1907513(10)$

$0.1891751(5)$

$0.192165(1)$

$0.194282(1)$

$0.208590(1)$

$0.1715814(7)$

$0.185023(1)$

$0.1943666(8)$

$0.197224(2)$

$0.200332(1)$

$0.1822379(10)$

$0.1771742(9)$

$0.178741(5)$

$0.185663(1)$

$0.184773(1)$

$0.2208207(8)$

$0.203469(1)$

$0.1682510(7)$

$0.1851302(5)$

$0.216754(2)$

$0.2006170(3)$

$P_{1 \mathrm{O}} / P_{\mathrm{X}}$

0.61461

0.61417

0.62165

0.61266

0.63072

0.61437

0.61255

0.61317

0.61358

0.61227

0.61296

0.61364

0.61243

0.62208

0.63087

0.61248

0.61289

0.62154

0.6127

0.61554

0.61167

0.63153

0.61016

0.61198

0.61303

0.62149

0.61246

0.61445

0.61464

0.61433

0.61341

0.6126

0.60949

0.63065

0.61437

0.61324

0.63095

0.61341

0.619

0.6129

0.62259

0.61256

0.62875

0.61375

0.61235

0.61291

0.62192

0.63172

0.61421

0.61373

0.61349

0.63725

0.61383

0.60899

0.6304

0.6125

0.6119

0.61403 0.61485

$A_{10}[\mathrm{mag}]$

$0.1325(4)$

$0.1279(4)$

$0.1226(2)$

$0.1226(2)$

$0.1226(2)$

$0.1432(7)$

$0.1373(4)$

$0.1205(3)$

$0.1092(3)$

$0.1233(4)$

$0.1431(3)$

$0.1247(2)$

$0.1200(2)$

$0.1200(2)$

$0.1200(2)$

$0.1025(2)$

$0.1202(2)$

$0.1202(2)$

$0.1276(5)$

$0.1195(2)$

$0.1354(4)$

$0.1379(2)$

$0.1539(2)$

$0.0885(3)$

$0.1477(2)$

$0.1245(4)$

$0.1245(4)$

0.0616(3)

$0.1534(3)$

$0.1325(2)$

$0.1317(1)$

$0.1365(3)$

$0.1270(5)$

$0.1155(5)$

$0.1376(4)$

$0.1248(2)$

$0.1253(2)$

$0.1253(2)$

$0.1232(4)$

$0.1249(1)$

$0.1249(1)$

$0.1175(3)$

$0.1319(2)$

$0.1362(2)$

$0.1264(4)$

$0.1249(2)$

$0.1249(2)$

$0.1249(2)$

$0.1263(3)$

$0.1293(5)$

$0.114(1)$

$0.114(1)$

$0.1312(5)$

$0.14652(10)$

$A_{\mathrm{X}} / A_{1 \mathrm{O}} \quad$ Remarks

0.023 cand.

0.019 RRd

$0.017 \quad 0.5 f_{x}$

0.015

0.011

0.014

0.018

0.015

0.018

0.081

0.011

0.026

0.015

0.011 
Table A1 - Continued from previous page

\begin{tabular}{c} 
ID \\
\hline OGLE-BLG-RRLYR-11249 \\
OGLE-BLG-RRLYR-11257 \\
OGLE-BLG-RRLYR-11260 \\
OGLE-BLG-RRLYR-11263 \\
\\
OGLE-BLG-RRLYR-11270 \\
OGLE-BLG-RRLYR-11281 \\
OGLE-BLG-RRLYR-11293 \\
OGLE-BLG-RRLYR-11299 \\
OGLE-BLG-RRLYR-11318 \\
OGLE-BLG-RRLYR-11339
\end{tabular}

OGLE-BLG-RRLYR-11348 OGLE-BLG-RRLYR-11384 OGLE-BLG-RRLYR-11394 OGLE-BLG-RRLYR-11440 OGLE-BLG-RRLYR-11451

OGLE-BLG-RRLYR-11457 OGLE-BLG-RRLYR-11466 OGLE-BLG-RRLYR-11474 OGLE-BLG-RRLYR-11490 OGLE-BLG-RRLYR-11497 OGLE-BLG-RRLYR-11503 OGLE-BLG-RRLYR-11533

OGLE-BLG-RRLYR-11539

OGLE-BLG-RRLYR-11547

OGLE-BLG-RRLYR-11559 OGLE-BLG-RRLYR-11564 OGLE-BLG-RRLYR-11575 OGLE-BLG-RRLYR-11594 OGLE-BLG-RRLYR-11599 OGLE-BLG-RRLYR-11615 OGLE-BLG-RRLYR-11621

OGLE-BLG-RRLYR-11641

OGLE-BLG-RRLYR-11671 OGLE-BLG-RRLYR-11673 OGLE-BLG-RRLYR-11682

OGLE-BLG-RRLYR-11684 OGLE-BLG-RRLYR-11687 OGLE-BLG-RRLYR-11716 OGLE-BLG-RRLYR-11726 OGLE-BLG-RRLYR-11728

OGLE-BLG-RRLYR-11744 OGLE-BLG-RRLYR-11761 OGLE-BLG-RRLYR-11789 OGLE-BLG-RRLYR-11843 OGLE-BLG-RRLYR-11882

\begin{tabular}{|c|c|c|c|c|c|}
\hline$P_{1 \mathrm{O}}[\mathrm{d}]$ & $P_{\mathrm{X}}[\mathrm{d}]$ & $P_{1 \mathrm{O}} / P_{\mathrm{X}}$ & $A_{10}[\mathrm{mag}]$ & $A_{\mathrm{X}} / A_{1 \mathrm{O}}$ & Remarks \\
\hline $0.30349768(4)$ & $0.1861912(10)$ & 0.61348 & $0.1284(3)$ & 0.011 & \\
\hline $0.31696424(4)$ & $0.194167(1)$ & 0.61258 & $0.1141(2)$ & 0.012 & $0.5 f_{x}$ \\
\hline $0.30636461(6)$ & $0.187669(1)$ & 0.61257 & $0.1260(4)$ & 0.018 & \\
\hline $0.35804243(5)$ & $0.2247024(7)$ & 0.62759 & $0.1348(2)$ & 0.016 & $0.5 f_{x}$ \\
\hline $0.35804243(5)$ & $0.2209425(7)$ & 0.61708 & $0.1348(2)$ & 0.014 & \\
\hline $0.2931183(1)$ & $0.182708(1)$ & 0.62333 & $0.1322(10)$ & 0.024 & cand. \\
\hline $0.31538481(4)$ & $0.195059(1)$ & 0.61848 & $0.1266(3)$ & 0.009 & \\
\hline $0.28490771(4)$ & $0.175366(1)$ & 0.61552 & $0.1399(3)$ & 0.012 & \\
\hline $0.31962796(6)$ & $0.195443(1)$ & 0.61147 & $0.1249(4)$ & 0.014 & cand. \\
\hline $0.28426071(2)$ & $0.1745236(5)$ & 0.61396 & $0.1474(2)$ & 0.016 & \\
\hline $0.30943246(4)$ & $0.1898592(6)$ & 0.61357 & $0.1190(2)$ & 0.022 & $0.5 f_{x}$ \\
\hline $0.30943246(4)$ & $0.192407(1)$ & 0.62181 & $0.1190(2)$ & 0.011 & \\
\hline $0.30943246(4)$ & $0.195337(2)$ & 0.63128 & $0.1190(2)$ & 0.009 & \\
\hline $0.3075237(2)$ & $0.1884152(6)$ & 0.61269 & $0.1217(9)$ & 0.011 & \\
\hline $0.35041096(6)$ & $0.216487(1)$ & 0.61781 & $0.1239(3)$ & 0.019 & \\
\hline $0.39402890(6)$ & $0.243397(2)$ & 0.61771 & $0.1486(7)$ & 0.011 & cand. \\
\hline $0.29242966(5)$ & 0.179599(1) & 0.61416 & $0.1269(4)$ & 0.013 & \\
\hline $0.32962549(2)$ & $0.203521(1)$ & 0.61743 & $0.1232(3)$ & 0.008 & \\
\hline $0.32962549(2)$ & $0.205274(2)$ & 0.62275 & $0.1232(3)$ & 0.006 & \\
\hline $0.31576255(2)$ & $0.199453(1)$ & 0.63166 & $0.1402(1)$ & 0.005 & \\
\hline $0.30935669(6)$ & $0.1894216(10)$ & 0.61231 & $0.1273(4)$ & 0.016 & \\
\hline $0.32585655(7)$ & $0.202544(1)$ & 0.62157 & $0.1247(4)$ & 0.013 & \\
\hline $0.3000787(1)$ & $0.183958(1)$ & 0.61303 & $0.1323(8)$ & 0.029 & cand. \\
\hline $0.30793498(5)$ & $0.188773(1)$ & 0.61303 & $0.1274(5)$ & 0.017 & \\
\hline $0.31097370(2)$ & $0.1902592(4)$ & 0.61182 & $0.1321(1)$ & 0.017 & \\
\hline $0.27108944(3)$ & $0.1685411(7)$ & 0.62172 & $0.0908(2)$ & 0.015 & \\
\hline $0.27108944(3)$ & $0.1662300(7)$ & 0.61319 & $0.0908(2)$ & 0.014 & \\
\hline $0.33389316(3)$ & $0.2107214(5)$ & 0.6311 & $0.1328(2)$ & 0.014 & $0.5 f_{x}$ \\
\hline $0.33389316(3)$ & $0.2092792(9)$ & 0.62678 & $0.1328(2)$ & 0.01 & \\
\hline $0.30619293(2)$ & $0.1871226(6)$ & 0.61113 & $0.1334(1)$ & 0.018 & \\
\hline $0.30619293(2)$ & $0.190180(1)$ & 0.62111 & $0.1334(1)$ & 0.01 & \\
\hline $0.30619293(2)$ & $0.193095(1)$ & 0.63063 & $0.1334(1)$ & 0.009 & \\
\hline $0.30529110(5)$ & $0.1873351(10)$ & 0.61363 & $0.1316(3)$ & 0.015 & \\
\hline $0.28032371(6)$ & $0.172018(1)$ & 0.61364 & $0.1213(4)$ & 0.016 & \\
\hline $0.29661620(5)$ & $0.181800(1)$ & 0.61291 & $0.1271(4)$ & 0.028 & \\
\hline $0.30000209(9)$ & $0.184129(1)$ & 0.61376 & $0.1110(5)$ & 0.018 & \\
\hline $0.35037715(9)$ & $0.2197132(6)$ & 0.62708 & $0.1101(4)$ & 0.015 & \\
\hline $0.36799364(4)$ & $0.2211492(8)$ & 0.60096 & $0.1596(2)$ & 0.006 & cand. \\
\hline $0.31723543(4)$ & $0.1971649(10)$ & 0.62151 & $0.1184(2)$ & 0.014 & \\
\hline $0.31723543(4)$ & $0.194482(1)$ & 0.61305 & $0.1184(2)$ & 0.013 & \\
\hline $0.31723543(4)$ & $0.2003121(10)$ & 0.63143 & $0.1184(2)$ & 0.014 & \\
\hline $0.32268682(3)$ & $0.2034747(6)$ & 0.63056 & $0.1354(2)$ & 0.015 & $0.5 f_{x}$ \\
\hline $0.32268682(3)$ & $0.203186(1)$ & 0.62967 & $0.1354(2)$ & 0.008 & \\
\hline $0.31992500(6)$ & $0.195872(1)$ & 0.61224 & $0.1071(3)$ & 0.018 & \\
\hline $0.25312719(2)$ & $0.1551514(6)$ & 0.61294 & $0.1331(2)$ & 0.011 & \\
\hline $0.3284178(4)$ & $0.208532(1)$ & 0.63496 & $0.1313(2)$ & 0.007 & $0.5 f_{x}$ \\
\hline $0.3284178(4)$ & $0.201097(1)$ & 0.61232 & $0.1313(2)$ & 0.006 & \\
\hline $0.3284178(4)$ & $0.204639(2)$ & 0.62311 & $0.1313(2)$ & 0.006 & \\
\hline $0.30178720(5)$ & $0.185309(1)$ & 0.61404 & $0.1324(4)$ & 0.014 & \\
\hline $0.29671591(3)$ & $0.1817651(10)$ & 0.61259 & $0.1431(2)$ & 0.011 & \\
\hline $0.28835654(4)$ & $0.176892(1)$ & 0.61345 & $0.1379(4)$ & 0.015 & \\
\hline $0.30194578(8)$ & $0.184807(1)$ & 0.61205 & $0.1212(5)$ & 0.019 & \\
\hline $0.31518348(3)$ & $0.193172(1)$ & 0.61289 & $0.1204(1)$ & 0.009 & $0.5 f_{x}$ \\
\hline $0.31518348(3)$ & $0.198961(1)$ & 0.63125 & $0.1204(1)$ & 0.008 & \\
\hline $0.33668044(6)$ & $0.206833(1)$ & 0.61433 & $0.1313(4)$ & 0.013 & \\
\hline $0.30881091(5)$ & $0.1893119(7)$ & 0.61304 & $0.1291(3)$ & 0.018 & \\
\hline $0.26796412(2)$ & $0.1639653(5)$ & 0.61189 & $0.1145(1)$ & 0.016 & \\
\hline $0.29510274(2)$ & $0.1804165(6)$ & 0.61137 & $0.1339(2)$ & 0.014 & \\
\hline $0.33676330(5)$ & $0.211768(1)$ & 0.62883 & $0.1190(3)$ & 0.012 & $0.5 f_{x}$ \\
\hline
\end{tabular}

Continued on next page 
Table A1 - Continued from previous page

ID
OGLE-BLG-RRLYR-11888
OGLE-BLG-RRLYR-11911
OGLE-BLG-RRLYR-11913

OGLE-BLG-RRLYR-11917
OGLE-BLG-RRLYR-11918
OGLE-BLG-RRLYR-11929
OGLE-BLG-RRLYR-11937
OGLE-BLG-RRLYR-11940
OGLE-BLG-RRLYR-11942
OGLE-BLG-RRLYR-11944

OGLE-BLG-RRLYR-11945 OGLE-BLG-RRLYR-11946 OGLE-BLG-RRLYR-11950

OGLE-BLG-RRLYR-11951 OGLE-BLG-RRLYR-11971 OGLE-BLG-RRLYR-11976

\section{OGLE-BLG-RRLYR-11980}

OGLE-BLG-RRLYR-11981 OGLE-BLG-RRLYR-11999 OGLE-BLG-RRLYR-12016 OGLE-BLG-RRLYR-12029 OGLE-BLG-RRLYR-12037 OGLE-BLG-RRLYR-12050 OGLE-BLG-RRLYR-12057 OGLE-BLG-RRLYR-12065 OGLE-BLG-RRLYR-12071

OGLE-BLG-RRLYR-12099 OGLE-BLG-RRLYR-12113 OGLE-BLG-RRLYR-12124 OGLE-BLG-RRLYR-12127 OGLE-BLG-RRLYR-12154 OGLE-BLG-RRLYR-12160 OGLE-BLG-RRLYR-12182 OGLE-BLG-RRLYR-12226 OGLE-BLG-RRLYR-12235 OGLE-BLG-RRLYR-12247 OGLE-BLG-RRLYR-12256 OGLE-BLG-RRLYR-12257 OGLE-BLG-RRLYR-12261

OGLE-BLG-RRLYR-12263 OGLE-BLG-RRLYR-12267

OGLE-BLG-RRLYR-12286 OGLE-BLG-RRLYR-12295 OGLE-BLG-RRLYR-12299 OGLE-BLG-RRLYR-12306 OGLE-BLG-RRLYR-12311 OGLE-BLG-RRLYR-12315

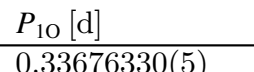

$0.29085810(5)$

$0.27016160(4)$

$0.31835441(3)$

$0.31835441(3)$

$0.31835441(3)$

$0.31332788(6)$

$0.32338881(6)$

$0.31588788(5)$

$0.31588788(5)$

$0.29158962(3)$

$0.29745121(3)$

$0.22410678(4)$

$0.32852428(4)$

$0.32852428(4)$

$0.33397005(4)$

$0.27819496(2)$

$0.30609485(2)$

$0.30609485(2)$

$0.33150132(3)$

$0.270646470(10)$

$0.31581013(4)$

$0.31581013(4)$

$0.31581013(4)$

$0.31660450(3)$

$0.31660450(3)$

$0.43938460(5)$

$0.30944767(3)$

$0.31169668(9)$

$0.32052172(3)$

$0.31908920(4)$

$0.32933833(2)$

$0.3172949(1)$

$0.34050387(7)$

$0.33047806(7)$

$0.33047806(7)$

$0.29871627(3)$

$0.31058606(2)$

$0.31224552(3)$

$0.28870492(3)$

$0.28682675(4)$

$0.30122636(3)$

$0.29601801(8)$

$0.29940839(6)$

$0.30416688(7)$

$0.27994410(4)$

$0.3184930(1)$

$0.31832443(4)$

$0.30506798(2)$

$0.30506798(2)$

$0.32622315(3)$

$0.33840524(10)$

$0.33840524(10)$

$0.30980777(6)$

$0.29322906(4)$

$0.282418370(10)$

$0.29727054(8)$

$0.28550049(2)$

$0.286810500(10)$
$P_{\mathrm{X}}[\mathrm{d}]$ $0.212870(2)$

$0.1787709(7)$

$0.1654728(5)$

$0.1978616(6)$

$0.1952227(9)$

$0.2009750(8)$

$0.192038(1)$

$0.1981339(6)$

$0.196545(1)$

$0.193735(1)$

0.179031(1)

$0.1826779(8)$

$0.1378743(5)$

$0.201154(1)$

$0.207394(1)$

$0.2097895(6)$

$0.1708588(7)$

$0.1890767(10)$

$0.190494(1)$

$0.208033(1)$

$0.1640809(4)$

$0.1936398(9)$

0.196329(1)

$0.199341(1)$

$0.1968910(6)$

$0.1941198(6)$

$0.270908(3)$

$0.189603(1)$

$0.193673(1)$

$0.1990895(6)$

$0.2012350(8)$

$0.208283(1)$

$0.194373(1)$

$0.2099190(9)$

$0.207791(1)$

$0.208436(2)$

$0.1832166(5)$

$0.1892545(8)$

$0.1940110(9)$

$0.1773870(10)$

$0.1757207(9)$

$0.1851108(7)$

$0.1812245(7)$

$0.1836628(4)$

$0.186578(1)$

$0.172647(1)$

$0.2009065(7)$

$0.200860(1)$

$0.1868364(3)$

$0.1925181(8)$

$0.2002165(7)$

$0.2133009(8)$

$0.2130711(3)$

$0.189739(1)$

$0.1796343(7)$

$0.1733697(2)$

$0.1826886(6)$

$0.1748721(7)$

$0.1763490(6)$

$P_{1 \mathrm{O}} / P_{\mathrm{X}}$
$A_{10}[\mathrm{mag}]$ 3211 0.61463 0.6125 0.62151 0.61322 0.63129 0.6129 0.61268 0.6222 0.6133 0.61398

0.61414

0.61522

0.6123

0.63129

0.62817

0.61417

0.61771

0.62234

0.62755

0.60626

0.61315

0.62167

0.63121

0.62188

0.61313

0.61656

0.61271

0.62135

0.62114

0.63065

0.63243

0.61259

0.6165

0.62876

0.63071

0.61335

0.60935

0.62134

0.61442

0.61264

0.61452

0.61221

0.61342

0.61341

0.61672

0.6308

0.63099

0.61244

0.63107

0.61374

0.63031

0.62963

0.61244

0.61261

0.61388

0.61455

0.61251 0.61486

$0.1217(3)$

$0.1234(1)$

0.1234(1)

$0.1234(1)$

$0.1229(3)$

$0.1207(3)$

$0.1190(3)$

$0.1272(3)$

0.0766(3)

$0.1351(3)$

$0.1351(3)$

$0.1094(2)$

$0.1488(2)$

$0.1327(1)$

$0.1327(1)$

$0.1428(3)$

$0.1433(1)$

$0.1158(2)$

$0.1158(2)$

$0.1158(2)$

$0.1240(2)$

$0.1240(2)$

$0.1143(1)$

$0.1470(3)$

$0.1232(6)$

$0.1266(2)$

$0.1273(3)$

$0.1190(3) \quad 0.011$

$0.1210(4) \quad 0.024$

0.01

0.016

0.014

0.014

0.014

0.017

0.017

0.013

$0.1372(3) \quad 0.01$

0.02

0.031

0.007

0.007

0.02

0.009

0.009

0.008

0.01

0.01

0.015

0.01

0.011

0.016

0.016

0.008

0.008

0.014

0.017

$0.1308(8) \quad 0.033$

$0.1150(3) \quad 0.011$

$0.1137(4) \quad 0.018$

$0.1137(4) \quad 0.013$

$0.1327(3) \quad 0.014$

$0.1303(1) \quad 0.012$

$0.1187(2) \quad 0.013$

$0.1438(3) \quad 0.012$ 
Table A1 - Continued from previous page

\begin{tabular}{c} 
ID \\
\hline OGLE-BLG-RRLYR-12324 \\
OGLE-BLG-RRLYR-12335 \\
OGLE-BLG-RRLYR-12355 \\
OGLE-BLG-RRLYR-12363 \\
OGLE-BLG-RRLYR-12369 \\
OGLE-BLG-RRLYR-12375 \\
OGLE-BLG-RRLYR-12406 \\
OGLE-BLG-RRLYR-12414 \\
OGLE-BLG-RRLYR-12421 \\
OGLE-BLG-RRLYR-12432 \\
OGLE-BLG-RRLYR-12434 \\
OGLE-BLG-RRLYR-12439 \\
OGLE-BLG-RRLYR-12445 \\
OGLE-BLG-RRLYR-12457 \\
OGLE-BLG-RRLYR-12467 \\
OGLE-BLG-RRLYR-12481 \\
OGLE-BLG-RRLYR-12492 \\
OGLE-BLG-RRLYR-12509 \\
OGLE-BLG-RRLYR-12528 \\
OGLE-BLG-RRLYR-12534 \\
OGLE-BLG-RRLYR-12539
\end{tabular}

OGLE-BLG-RRLYR-12549 OGLE-BLG-RRLYR-12567 OGLE-BLG-RRLYR-12570 OGLE-BLG-RRLYR-12574 OGLE-BLG-RRLYR-12582 OGLE-BLG-RRLYR-12640 OGLE-BLG-RRLYR-12646

OGLE-BLG-RRLYR-12653 OGLE-BLG-RRLYR-12663 OGLE-BLG-RRLYR-12672 OGLE-BLG-RRLYR-12686 OGLE-BLG-RRLYR-12697 OGLE-BLG-RRLYR-12723 OGLE-BLG-RRLYR-12769

OGLE-BLG-RRLYR-12776

OGLE-BLG-RRLYR-12823 OGLE-BLG-RRLYR-12828 OGLE-BLG-RRLYR-12829 OGLE-BLG-RRLYR-12831 OGLE-BLG-RRLYR-12845 OGLE-BLG-RRLYR-12878 OGLE-BLG-RRLYR-12883 OGLE-BLG-RRLYR-12921 OGLE-BLG-RRLYR-12944 OGLE-BLG-RRLYR-12951 OGLE-BLG-RRLYR-12972

OGLE-BLG-RRLYR-12984 OGLE-BLG-RRLYR-12989
$P_{10}[\mathrm{~d}]$ $0.29531340(7)$

$0.4605875(4)$

$P_{\mathrm{X}}[\mathrm{d}]$

$0.35181130(9)$

$0.31900473(7)$

$0.26545416(3)$

$0.31915968(8)$

$0.28154412(8)$

$0.27819884(2)$

$0.33805606(3)$

$0.33805606(3)$

$0.287155290(10)$

$0.29426773(2)$

$0.295584400(10)$

$0.34370588(4)$

$0.29025537(2)$

$0.32027826(4)$

$0.32027826(4)$

$0.30570649(3)$

$0.32190925(7)$

$0.34522697(8)$

$0.32416290(4)$

$0.26476896(5)$

$0.30799589(4)$

$0.30799589(4)$

$0.31012872(7)$

$0.32336079(4)$

$0.29167537(5)$

$0.24535126(2)$

$0.31321036(4)$

$0.31421950(3)$

$0.31687062(3)$

$0.31687062(3)$

$0.2719433(1)$

$0.31303208(7)$

$0.235402040(10)$

$0.29624091(2)$

$0.37628587(7)$

$0.249187140(10)$

$0.31341085(2)$

$0.31341085(2)$

$0.31341085(2)$

$0.31535057(2)$

$0.31535057(2)$

$0.31535057(2)$

$0.29009132(5)$

$0.32729450(5)$

$0.32213541(8)$

$0.28804298(5)$

$0.274383150(10)$

$0.268720250(10)$

$0.28626854(5)$

$0.28454394(3)$

$0.30277364(3)$

$0.23228839(2)$

$0.32094619(3)$

$0.32094619(3)$

$0.32094619(3)$

$0.36126930(2)$ $0.30680146(8)$

$0.180900(1)$
(3)

$0.216186(1)$

$0.1982551(6)$

$0.1624755(4)$

$0.198368(1)$

$0.172529(1)$

$0.1708247(6)$

$0.2130284(9)$

$0.212771(1)$

$0.1754099(5)$

$0.1807983(5)$

$0.1815171(3)$

$0.210842(1)$

$0.1776622(5)$

$0.1991096(9)$

$0.1961614(8)$

$0.1874013(3)$

$0.196973(2)$

$0.211293(1)$

$0.2049759(9)$

$0.1625541(8)$

$0.187221(1)$

$0.1927168(10)$

0.190781(1)

$0.1980317(9)$

$0.1790998(8)$

$0.1505015(6)$

$0.1921434(10)$

$0.1983429(9)$

$0.19705(1)$

$0.1941832(8)$

$0.171915(1)$

$0.191935(5)$

$0.144570(1)$

$0.1820252(6)$

$0.229622(2)$

0.1532521(3)

$0.1921129(6)$

$0.1950164(7)$

$0.1978961(8)$

0.1936490(3)

$0.1959375(7)$

$0.1990300(5)$

$0.178470(1)$

$0.200351(1)$

$0.203257(2)$

$0.176666(1)$

$0.165981(2)$

$0.161104(1)$

$0.175844(1)$

$0.174665(1)$

$0.1858722(1)$

$0.1425174(6)$

$0.1965611(8)$

$0.1992075(9)$

$0.202334(1)$

$0.2267288(10)$

$0.188014(1)$
$P_{1 \mathrm{O}} / P_{\mathrm{X}}$

0.63462

0.61449

0.62148

0.61207

0.62153

0.6128

0.61404

0.63016

0.6294

0.61085

0.6144

0.6141

0.61344

0.61209

0.62168

0.61247

0.61301

0.61189

0.61204

0.63232

0.61395

0.60787

0.62571

0.61517

0.61242

0.61404

0.61341

0.61346

0.63122

0.62186

0.61282

0.63217

0.61315

0.61414

0.61445

0.61023

0.61501

0.61297

0.62224

0.63143

0.61408

0.62133

0.63114

0.61522

0.61214

0.63097

0.61333

0.60492

0.59952

0.61426

0.61384

0.6139

0.61354

0.61244

0.62069

0.63043

0.62759

0.61282
$A_{10}[\mathrm{mag}]$ $0.1382(6) \quad 0.02$ $0.0973(10) \quad 0.028$ $0.1432(6) \quad 0.022$ $0.1243(4) \quad 0.014$ $0.1125(2) \quad 0.022$ $0.1269(5) \quad 0.02$ $0.1105(5)$ $0.1476(2)$ 0.022 0.012 0.014 $0.1141(2) \quad 0.01$ $0.08476(7) \quad 0.01$ $0.1285(2) \quad 0.02$ $0.1449(1) \quad 0.021$ $0.1214(2) \quad 0.008$ $0.1443(2) \quad 0.015$ $0.1180(2) \quad 0.02$ $0.1180(2) \quad 0.015$ $0.1319(2) \quad 0.045$ $0.1251(4) \quad 0.012$ $0.1283(4) \quad 0.009$ $0.1249(2) \quad 0.01$ $0.1071(4) \quad 0.014$ $0.1197(3) \quad 0.009$ $0.1197(3) \quad 0.01$ $0.1248(4) \quad 0.016$ $0.1232(2) \quad 0.011$ $0.1381(4) \quad 0.021$ $0.1216(2) \quad 0.011$ $0.1290(3) \quad 0.013$ $0.1117(1) \quad 0.012$ $0.1143(2) \quad 0.012$ $0.1143(2) \quad 0.01$ $0.1255(4) \quad 0.023$ $0.1209(4) \quad 0.017$ $0.0966(1) \quad 0.033$ $0.1356(1) \quad 0.026$ $0.1039(2) \quad 0.008$ $0.1240(1) \quad 0.016$ $0.1254(1) \quad 0.021$ $0.1254(1) \quad 0.013$ $0.1254(1) \quad 0.01$ $0.1302(1) \quad 0.01$ $0.1302(1) \quad 0.007$ $0.1302(1) \quad 0.007$ $0.1529(6) \quad 0.017$ $0.1227(3) \quad 0.011$ $0.1268(5) \quad 0.014$ $0.1269(7) \quad 0.023$ $0.1477(1) \quad 0.007$ $0.1550(1) \quad 0.005$ $0.1282(4) \quad 0.012$ $0.1351(3) \quad 0.016$ $0.1286(2) \quad 0.039$ $0.1177(2) \quad 0.014$ $0.1208(1) \quad 0.012$ $0.1208(1) \quad 0.011$ $0.1208(1) \quad 0.009$ $0.1292(2) \quad 0.014$ $0.1346(5) \quad 0.012$
Remarks

cand.

$0.5 f_{x}$

$0.5 f_{x}$

cand.

$0.5 f_{x}$

$0.5 f_{x}$

$0.5 f_{x}$

Continued on next page 
Table A1 - Continued from previous page

\begin{tabular}{c} 
ID \\
\hline OGLE-BLG-RRLYR-13003 \\
OGLE-BLG-RRLYR-13008 \\
OGLE-BLG-RRLYR-13021 \\
OGLE-BLG-RRLYR-13071 \\
OGLE-BLG-RRLYR-13099 \\
OGLE-BLG-RRLYR-13117 \\
OGLE-BLG-RRLYR-13129 \\
OGLE-BLG-RRLYR-13136 \\
OGLE-BLG-RRLYR-13153 \\
OGLE-BLG-RRLYR-13156
\end{tabular}

OGLE-BLG-RRLYR-13169 OGLE-BLG-RRLYR-13172 OGLE-BLG-RRLYR-13184 OGLE-BLG-RRLYR-13194 OGLE-BLG-RRLYR-13198 OGLE-BLG-RRLYR-13204 OGLE-BLG-RRLYR-13247 OGLE-BLG-RRLYR-13253 OGLE-BLG-RRLYR-13256 OGLE-BLG-RRLYR-13269 OGLE-BLG-RRLYR-13328 OGLE-BLG-RRLYR-13332 OGLE-BLG-RRLYR-13400

OGLE-BLG-RRLYR-13401 OGLE-BLG-RRLYR-13422 OGLE-BLG-RRLYR-13455 OGLE-BLG-RRLYR-13459 OGLE-BLG-RRLYR-13472 OGLE-BLG-RRLYR-13518 OGLE-BLG-RRLYR-13536 OGLE-BLG-RRLYR-13540 OGLE-BLG-RRLYR-13550 OGLE-BLG-RRLYR-13567

OGLE-BLG-RRLYR-13648 OGLE-BLG-RRLYR-13666 OGLE-BLG-RRLYR-13708

OGLE-BLG-RRLYR-13717 OGLE-BLG-RRLYR-13721 OGLE-BLG-RRLYR-13724 OGLE-BLG-RRLYR-13740 OGLE-BLG-RRLYR-13758 OGLE-BLG-RRLYR-13771 OGLE-BLG-RRLYR-13870 OGLE-BLG-RRLYR-13885 OGLE-BLG-RRLYR-13889 OGLE-BLG-RRLYR-13892 OGLE-BLG-RRLYR-13981 OGLE-BLG-RRLYR-13989 OGLE-BLG-RRLYR-14013 OGLE-BLG-RRLYR-14029 OGLE-BLG-RRLYR-14031 OGLE-BLG-RRLYR-14098

\begin{tabular}{|c|c|c|c|c|c|}
\hline$P_{10}[\mathrm{~d}]$ & $P_{\mathrm{X}}[\mathrm{d}]$ & $P_{1 \mathrm{O}} / P_{\mathrm{X}}$ & $A_{1 \mathrm{O}}[\mathrm{mag}]$ & $A_{\mathrm{X}} / A_{1 \mathrm{O}}$ & Remarks \\
\hline $0.30888388(8)$ & $0.189648(1)$ & 0.61398 & $0.1236(5)$ & 0.026 & cand. \\
\hline $0.34044133(9)$ & $0.209252(2)$ & 0.61465 & $0.1441(4)$ & 0.019 & \\
\hline $0.34044133(9)$ & $0.212040(5)$ & 0.62284 & $0.1441(4)$ & 0.015 & \\
\hline $0.28573086(2)$ & $0.1747994(8)$ & 0.61176 & $0.1274(2)$ & 0.009 & \\
\hline $0.3125351(2)$ & $0.191635(2)$ & 0.61316 & $0.1213(8)$ & 0.042 & cand. \\
\hline $0.28071469(3)$ & $0.1721670(8)$ & 0.61332 & $0.1497(2)$ & 0.01 & \\
\hline $0.32160835(8)$ & $0.199955(2)$ & 0.62173 & $0.1275(5)$ & 0.018 & cand. \\
\hline $0.3114412(1)$ & $0.1908630(8)$ & 0.61284 & $0.1209(3)$ & 0.025 & \\
\hline $0.27939720(4)$ & $0.1712980(10)$ & 0.6131 & $0.1130(3)$ & 0.016 & cand. \\
\hline $0.3224849(2)$ & $0.2042081(10)$ & 0.63323 & $0.0674(5)$ & 0.042 & $0.5 f_{x}$ \\
\hline $0.31633834(3)$ & $0.193936(5)$ & 0.61307 & $0.1238(2)$ & 0.015 & $0.5 f_{x}$ \\
\hline $0.31633834(3)$ & $0.196753(3)$ & 0.62197 & $0.1238(2)$ & 0.008 & \\
\hline $0.31633834(3)$ & $0.19973(3)$ & 0.63138 & $0.1238(2)$ & 0.008 & \\
\hline $0.29192497(6)$ & $0.1795010(9)$ & 0.61489 & $0.1320(5)$ & 0.022 & \\
\hline $0.34356909(3)$ & $0.210475(1)$ & 0.61261 & $0.1448(2)$ & 0.007 & \\
\hline $0.31028552(5)$ & $0.189819(1)$ & 0.61176 & $0.1312(4)$ & 0.021 & \\
\hline $0.22781277(6)$ & $0.1399038(5)$ & 0.61412 & $0.0874(6)$ & 0.041 & \\
\hline $0.32174412(5)$ & $0.203208(2)$ & 0.63159 & $0.1283(3)$ & 0.011 & $\mathrm{RRd}, 0.5 f_{x}$ \\
\hline $0.274348180(10)$ & $0.1687579(6)$ & 0.61512 & $0.1363(1)$ & 0.009 & \\
\hline $0.29538529(8)$ & $0.181518(1)$ & 0.61451 & $0.1264(5)$ & 0.024 & \\
\hline $0.32589718(4)$ & $0.205554(1)$ & 0.63073 & $0.1200(4)$ & 0.021 & $\mathrm{bl}$ \\
\hline $0.28843329(4)$ & $0.1765769(3)$ & 0.61219 & $0.1288(2)$ & 0.019 & \\
\hline $0.29716033(3)$ & $0.1817754(5)$ & 0.61171 & $0.1307(3)$ & 0.033 & \\
\hline $0.31800927(6)$ & $0.194913(2)$ & 0.61292 & $0.1172(3)$ & 0.02 & $\mathrm{bl}$ \\
\hline $0.22839511(3)$ & $0.1405923(8)$ & 0.61557 & $0.1296(3)$ & 0.01 & cand. \\
\hline $0.31896055(6)$ & $0.201170(1)$ & 0.6307 & $0.1244(4)$ & 0.015 & \\
\hline $0.31896055(6)$ & $0.195499(1)$ & 0.61293 & $0.1244(4)$ & 0.015 & \\
\hline $0.30024725(5)$ & $0.1838344(7)$ & 0.61228 & $0.1334(4)$ & 0.021 & \\
\hline $0.30211517(3)$ & $0.1849646(9)$ & 0.61223 & $0.1319(3)$ & 0.017 & \\
\hline $0.31186177(8)$ & $0.191189(1)$ & 0.61306 & $0.1160(5)$ & 0.022 & \\
\hline $0.28662699(3)$ & $0.1755890(10)$ & 0.6126 & $0.1214(3)$ & 0.012 & cand. \\
\hline $0.28588058(5)$ & $0.1751567(10)$ & 0.61269 & $0.1417(8)$ & 0.025 & \\
\hline $0.28585552(5)$ & $0.1755654(10)$ & 0.61418 & $0.1419(4)$ & 0.013 & \\
\hline $0.23177010(4)$ & $0.1427053(2)$ & 0.61572 & $0.1025(4)$ & 0.017 & \\
\hline $0.31699113(3)$ & $0.200027(1)$ & 0.63102 & $0.1430(3)$ & 0.009 & \\
\hline $0.32117112(6)$ & $0.196798(1)$ & 0.61275 & $0.1291(4)$ & 0.015 & \\
\hline $0.30632006(5)$ & $0.187580(1)$ & 0.61237 & $0.1344(4)$ & 0.018 & $\mathrm{bl}$ \\
\hline $0.30632006(5)$ & $0.190237(1)$ & 0.62104 & $0.1344(4)$ & 0.015 & \\
\hline $0.30632006(5)$ & $0.193192(1)$ & 0.63069 & $0.1344(4)$ & 0.015 & \\
\hline $0.30634813(4)$ & $0.1879412(8)$ & 0.61349 & $0.1338(3)$ & 0.013 & \\
\hline $0.30495950(6)$ & $0.192597(1)$ & 0.63155 & $0.1260(4)$ & 0.022 & $\mathrm{RRd}, 0.5 f_{x}$ \\
\hline $0.31371432(4)$ & $0.192259(1)$ & 0.61285 & $0.1249(3)$ & 0.01 & \\
\hline $0.31371432(4)$ & $0.195183(1)$ & 0.62217 & $0.1249(3)$ & 0.014 & \\
\hline $0.24396592(5)$ & $0.1494468(6)$ & 0.61257 & $0.1062(4)$ & 0.024 & \\
\hline $0.3393893(6)$ & $0.215822(2)$ & 0.63591 & $0.162(5)$ & 0.142 & $\mathrm{RRd}$ \\
\hline $0.28331525(4)$ & $0.173741(1)$ & 0.61324 & $0.1496(4)$ & 0.014 & \\
\hline $0.32459764(7)$ & $0.205000(1)$ & 0.63155 & $0.1242(5)$ & 0.021 & $0.5 f_{x}$ \\
\hline $0.29702302(4)$ & $0.184318(1)$ & 0.62055 & $0.1269(3)$ & 0.009 & cand. \\
\hline $0.35469654(4)$ & $0.21695(1)$ & 0.61165 & $0.1281(5)$ & 0.012 & \\
\hline $0.37661917(7)$ & $0.229416(2)$ & 0.60915 & $0.1446(4)$ & 0.01 & cand. \\
\hline $0.25128493(8)$ & $0.151945(1)$ & 0.60467 & $0.1015(6)$ & 0.025 & \\
\hline $0.28088012(3)$ & $0.1721219(4)$ & 0.61279 & $0.1481(4)$ & 0.018 & \\
\hline $0.28729088(5)$ & $0.1760587(7)$ & 0.61282 & $0.1359(5)$ & 0.024 & \\
\hline $0.30351522(6)$ & $0.1855141(9)$ & 0.61122 & $0.1237(4)$ & 0.021 & \\
\hline $0.28837244(3)$ & $0.177357(1)$ & 0.61503 & $0.1382(6)$ & 0.015 & $0.5 f_{x}$ \\
\hline $0.31991585(6)$ & $0.198815(1)$ & 0.62146 & $0.1043(3)$ & 0.014 & cand. \\
\hline $0.31798614(6)$ & $0.194845(1)$ & 0.61275 & $0.1164(3)$ & 0.016 & $\mathrm{RRd}$ \\
\hline $0.42976527(2)$ & $0.2657970(5)$ & 0.61847 & $0.1183(5)$ & 0.018 & $\mathrm{RRd}$ \\
\hline $0.31356509(6)$ & $0.191782(2)$ & 0.61162 & $0.1283(4)$ & 0.014 & cand. \\
\hline
\end{tabular}


Table A1 - Continued from previous page

\begin{tabular}{|c|c|c|c|c|c|c|}
\hline ID & $P_{1 \mathrm{O}}[\mathrm{d}]$ & $P_{\mathrm{X}}[\mathrm{d}]$ & $P_{1 \mathrm{O}} / P_{\mathrm{X}}$ & $A_{10}[\mathrm{mag}]$ & $A_{\mathrm{X}} / A_{1 \mathrm{O}}$ & Remarks \\
\hline OGLE-BLG-RRLYR-14120 & $0.29647439(9)$ & $0.182136(1)$ & 0.61434 & $0.1339(7)$ & 0.027 & \\
\hline OGLE-BLG-RRLYR-14130 & $0.26985234(9)$ & $0.172523(1)$ & 0.63932 & $0.0617(4)$ & 0.031 & cand. \\
\hline OGLE-BLG-RRLYR-14190 & $0.31324308(8)$ & $0.1921395(7)$ & 0.61339 & $0.1183(5)$ & 0.02 & \\
\hline OGLE-BLG-RRLYR-14263 & $0.3125081(1)$ & $0.194783(2)$ & 0.62329 & $0.1269(7)$ & 0.02 & \\
\hline OGLE-BLG-RRLYR-14292 & $0.3127711(1)$ & $0.199451(1)$ & 0.63769 & $0.131(1)$ & 0.053 & \\
\hline \multirow[t]{2}{*}{ OGLE-BLG-RRLYR-14301 } & $0.31423738(6)$ & $0.1954784(7)$ & 0.62207 & $0.1182(4)$ & 0.019 & \\
\hline & $0.31423738(6)$ & $0.1925942(4)$ & 0.61289 & $0.1182(4)$ & 0.016 & \\
\hline OGLE-BLG-RRLYR-14355 & $0.30234812(6)$ & $0.185092(1)$ & 0.61218 & $0.1189(4)$ & 0.014 & \\
\hline OGLE-BLG-RRLYR-14356 & $0.28429554(5)$ & $0.174815(1)$ & 0.61491 & $0.1439(5)$ & 0.017 & \\
\hline OGLE-BLG-RRLYR-14392 & $0.34160952(7)$ & $0.209830(2)$ & 0.61424 & $0.1494(5)$ & 0.024 & \\
\hline OGLE-BLG-RRLYR-14404 & $0.2453836(1)$ & $0.1501966(9)$ & 0.61209 & $0.0906(8)$ & 0.049 & cand. \\
\hline OGLE-BLG-RRLYR-14430 & $0.30560010(8)$ & $0.1867139(9)$ & 0.61097 & $0.1140(6)$ & 0.032 & \\
\hline OGLE-BLG-RRLYR-14462 & $0.27733361(2)$ & $0.170075(7)$ & 0.61325 & $0.1471(3)$ & 0.014 & \\
\hline OGLE-BLG-RRLYR-14467 & $0.26591246(5)$ & $0.1632927(3)$ & 0.61408 & $0.1084(3)$ & 0.024 & \\
\hline OGLE-BLG-RRLYR-14498 & $0.29350230(8)$ & $0.179632(1)$ & 0.61203 & $0.1301(6)$ & 0.023 & \\
\hline OGLE-BLG-RRLYR-14502 & $0.29470583(6)$ & $0.180837(1)$ & 0.61362 & $0.1292(4)$ & 0.016 & cand. \\
\hline OGLE-BLG-RRLYR-14510 & $0.29828126(8)$ & $0.1831414(9)$ & 0.61399 & $0.1274(6)$ & 0.029 & \\
\hline OGLE-BLG-RRLYR-14529 & $0.32719812(5)$ & $0.206723(2)$ & 0.6318 & $0.1312(3)$ & 0.01 & \\
\hline OGLE-BLG-RRLYR-14572 & $0.2224972(2)$ & $0.1366192(2)$ & 0.61403 & $0.0416(2)$ & 0.108 & \\
\hline OGLE-BLG-RRLYR-14609 & $0.3015711(2)$ & $0.1843810(10)$ & 0.6114 & $0.1281(4)$ & 0.02 & \\
\hline OGLE-BLG-RRLYR-14632 & $0.29903757(7)$ & $0.183557(1)$ & 0.61383 & $0.1346(5)$ & 0.018 & \\
\hline OGLE-BLG-RRLYR-14661 & $0.28632290(6)$ & $0.1807600(7)$ & 0.63132 & $0.1161(4)$ & 0.011 & \\
\hline OGLE-BLG-RRLYR-14669 & $0.30057306(10)$ & $0.1841269(10)$ & 0.61259 & $0.1303(6)$ & 0.031 & \\
\hline OGLE-BLG-RRLYR-14677 & $0.295202750(10)$ & $0.1811998(10)$ & 0.61381 & $0.1249(3)$ & 0.014 & \\
\hline OGLE-BLG-RRLYR-14687 & $0.27104250(5)$ & $0.1662652(7)$ & 0.61343 & $0.1097(4)$ & 0.035 & \\
\hline OGLE-BLG-RRLYR-14698 & $0.33536355(9)$ & $0.206085(1)$ & 0.61451 & $0.1276(5)$ & 0.018 & \\
\hline OGLE-BLG-RRLYR-14728 & $0.25342427(6)$ & $0.1553944(9)$ & 0.61318 & $0.0891(4)$ & 0.021 & cand. \\
\hline OGLE-BLG-RRLYR-14731 & $0.33148600(4)$ & $0.202922(1)$ & 0.61216 & $0.1452(3)$ & 0.018 & \\
\hline OGLE-BLG-RRLYR-14735 & $0.31390726(6)$ & $0.192481(2)$ & 0.61318 & $0.1304(4)$ & 0.015 & $0.5 f_{x}$ \\
\hline OGLE-BLG-RRLYR-14744 & $0.29256610(6)$ & $0.179124(1)$ & 0.61225 & $0.1354(5)$ & 0.018 & \\
\hline OGLE-BLG-RRLYR-14781 & $0.3540654(2)$ & $0.217199(2)$ & 0.61344 & $0.144(1)$ & 0.01 & \\
\hline OGLE-BLG-RRLYR-14826 & $0.29329512(5)$ & $0.180202(1)$ & 0.61441 & $0.1361(5)$ & 0.015 & cand. \\
\hline OGLE-BLG-RRLYR-14917 & $0.30481158(5)$ & $0.1867447(8)$ & 0.61266 & $0.1272(4)$ & 0.022 & \\
\hline OGLE-BLG-RRLYR-14920 & $0.23417782(7)$ & $0.1434989(6)$ & 0.61278 & $0.1005(6)$ & 0.023 & \\
\hline OGLE-BLG-RRLYR-14998 & $0.28782324(5)$ & $0.176488(1)$ & 0.61318 & $0.1415(4)$ & 0.016 & \\
\hline OGLE-BLG-RRLYR-15047 & $0.29429323(5)$ & $0.181090(1)$ & 0.61534 & $0.1245(3)$ & 0.016 & \\
\hline \multirow[t]{2}{*}{ OGLE-BLG-RRLYR-15072 } & $0.31749585(6)$ & $0.197340(1)$ & 0.62155 & $0.1242(3)$ & 0.015 & $0.5 f_{x}$ \\
\hline & $0.31749585(6)$ & $0.194507(2)$ & 0.61263 & $0.1242(3)$ & 0.01 & \\
\hline OGLE-BLG-RRLYR-15087 & $0.31771047(6)$ & $0.194775(1)$ & 0.61306 & $0.1110(4)$ & 0.016 & cand. \\
\hline OGLE-BLG-RRLYR-15093 & $0.25616024(3)$ & $0.1574156(8)$ & 0.61452 & $0.1366(3)$ & 0.012 & \\
\hline OGLE-BLG-RRLYR-15101 & $0.22759112(5)$ & $0.1395556(7)$ & 0.61319 & $0.1229(4)$ & 0.016 & cand. \\
\hline OGLE-BLG-RRLYR-15130 & $0.28628662(4)$ & $0.174935(1)$ & 0.61105 & $0.1505(5)$ & 0.011 & \\
\hline OGLE-BLG-RRLYR-15144 & $0.3110454(1)$ & $0.1907822(9)$ & 0.61336 & $0.1238(8)$ & 0.028 & \\
\hline OGLE-BLG-RRLYR-15185 & $0.32577414(8)$ & $0.199458(1)$ & 0.61226 & $0.1276(4)$ & 0.015 & \\
\hline OGLE-BLG-RRLYR-15220 & $0.29835484(9)$ & $0.182865(1)$ & 0.61291 & $0.1239(6)$ & 0.026 & cand. \\
\hline OGLE-BLG-RRLYR-15228 & $0.28294406(6)$ & $0.1736539(5)$ & 0.61374 & $0.1355(4)$ & 0.016 & \\
\hline OGLE-BLG-RRLYR-15237 & $0.28124366(4)$ & $0.1727120(8)$ & 0.6141 & $0.1374(4)$ & 0.02 & \\
\hline OGLE-BLG-RRLYR-15270 & $0.29980091(5)$ & $0.183681(1)$ & 0.61268 & $0.1209(4)$ & 0.016 & \\
\hline OGLE-BLG-RRLYR-15314 & $0.28516495(7)$ & $0.1741990(7)$ & 0.61087 & $0.1016(5)$ & 0.029 & \\
\hline OGLE-BLG-RRLYR-15345 & $0.30991656(5)$ & $0.190827(1)$ & 0.61574 & $0.1503(3)$ & 0.01 & cand. \\
\hline OGLE-BLG-RRLYR-15365 & $0.2519250(1)$ & $0.1544786(9)$ & 0.61319 & $0.0836(6)$ & 0.035 & \\
\hline OGLE-BLG-RRLYR-15427 & $0.33191744(6)$ & $0.203008(1)$ & 0.61162 & $0.1416(3)$ & 0.015 & \\
\hline OGLE-BLG-RRLYR-15451 & $0.29277057(5)$ & $0.179583(1)$ & 0.61339 & $0.1386(4)$ & 0.014 & cand. \\
\hline OGLE-BLG-RRLYR-15488 & $0.25705520(8)$ & $0.1571860(7)$ & 0.61149 & $0.1046(6)$ & 0.04 & \\
\hline OGLE-BLG-RRLYR-15534 & $0.2920620(2)$ & $0.179403(2)$ & 0.61426 & $0.1379(8)$ & 0.029 & \\
\hline OGLE-BLG-RRLYR-15544 & $0.31902847(10)$ & $0.196177(1)$ & 0.61492 & $0.1247(7)$ & 0.032 & \\
\hline OGLE-BLG-RRLYR-15624 & $0.30180310(5)$ & $0.1851445(8)$ & 0.61346 & $0.1265(4)$ & 0.018 & \\
\hline OGLE-BLG-RRLYR-15672 & $0.25924536(7)$ & $0.1594204(10)$ & 0.61494 & $0.1137(5)$ & 0.025 & \\
\hline OGLE-BLG-RRLYR-15791 & $0.30512111(7)$ & $0.187059(2)$ & 0.61306 & $0.1153(5)$ & 0.029 & \\
\hline
\end{tabular}


Table A1 - Continued from previous page

\begin{tabular}{|c|c|c|c|c|c|c|}
\hline ID & $P_{1 \mathrm{O}}[\mathrm{d}]$ & $P_{\mathrm{X}}[\mathrm{d}]$ & $P_{1 \mathrm{O}} / P_{\mathrm{X}}$ & $A_{10}[\mathrm{mag}]$ & $A_{\mathrm{X}} / A_{1 \mathrm{O}}$ & Remarks \\
\hline OGLE-BLG-RRLYR-15812 & $0.32555808(9)$ & $0.1994035(5)$ & 0.6125 & $0.1240(6)$ & 0.02 & \\
\hline OGLE-BLG-RRLYR-15873 & $0.29989436(6)$ & $0.184197(1)$ & 0.61421 & $0.1321(4)$ & 0.016 & \\
\hline OGLE-BLG-RRLYR-15906 & $0.31385133(7)$ & $0.192516(1)$ & 0.6134 & $0.1236(5)$ & 0.019 & \\
\hline OGLE-BLG-RRLYR-15978 & $0.28687856(4)$ & $0.176665(1)$ & 0.61582 & $0.1419(4)$ & 0.015 & \\
\hline OGLE-BLG-RRLYR-15997 & $0.29335126(7)$ & $0.179408(1)$ & 0.61158 & $0.1222(5)$ & 0.02 & cand. \\
\hline \multirow[t]{2}{*}{ OGLE-BLG-RRLYR-16002 } & $0.31423415(8)$ & $0.192680(1)$ & 0.61317 & $0.1223(5)$ & 0.018 & \\
\hline & $0.31423415(8)$ & $0.195342(1)$ & 0.62164 & $0.1223(5)$ & 0.017 & \\
\hline OGLE-BLG-RRLYR-16013 & $0.2996399(1)$ & $0.1838392(7)$ & 0.61353 & $0.1232(7)$ & 0.049 & \\
\hline OGLE-BLG-RRLYR-16018 & $0.30858443(7)$ & $0.189121(1)$ & 0.61287 & $0.1279(5)$ & 0.018 & cand. \\
\hline OGLE-BLG-RRLYR-16022 & $0.31745286(9)$ & $0.194568(1)$ & 0.6129 & $0.1242(6)$ & 0.023 & \\
\hline OGLE-BLG-RRLYR-16075 & $0.3148151(2)$ & $0.195779(1)$ & 0.62189 & $0.1227(9)$ & 0.035 & cand. \\
\hline OGLE-BLG-RRLYR-16077 & $0.30623737(6)$ & $0.187114(1)$ & 0.61101 & $0.1294(5)$ & 0.016 & \\
\hline OGLE-BLG-RRLYR-16111 & $0.30265397(7)$ & $0.185931(1)$ & 0.61434 & $0.1276(5)$ & 0.023 & cand. \\
\hline OGLE-BLG-RRLYR-16167 & $0.28842194(7)$ & $0.176884(1)$ & 0.61328 & $0.1270(5)$ & 0.024 & cand. \\
\hline OGLE-BLG-RRLYR-16205 & $0.3247993(1)$ & $0.199131(2)$ & 0.61309 & $0.1252(6)$ & 0.022 & \\
\hline OGLE-BLG-RRLYR-16246 & $0.30566956(7)$ & $0.188263(1)$ & 0.6159 & $0.1308(6)$ & 0.02 & \\
\hline OGLE-BLG-RRLYR-16268 & $0.25268392(4)$ & $0.1549075(9)$ & 0.61305 & $0.1350(4)$ & 0.019 & \\
\hline OGLE-BLG-RRLYR-16456 & $0.24949928(6)$ & $0.1528444(7)$ & 0.6126 & $0.0891(4)$ & 0.027 & \\
\hline OGLE-BLG-RRLYR-16517 & $0.3177013(1)$ & $0.194801(2)$ & 0.61316 & $0.1192(6)$ & 0.023 & cand. \\
\hline OGLE-BLG-RRLYR-17069 & $0.28025306(6)$ & $0.1719536(3)$ & 0.61357 & $0.1500(7)$ & 0.021 & cand. \\
\hline OGLE-BLG-RRLYR-17338 & $0.30568208(10)$ & $0.187157(1)$ & 0.61226 & $0.1333(8)$ & 0.032 & cand. \\
\hline OGLE-BLG-RRLYR-17471 & $0.28782131(3)$ & $0.1769667(10)$ & 0.61485 & $0.1365(8)$ & 0.027 & \\
\hline OGLE-BLG-RRLYR-17684 & $0.30364291(8)$ & $0.1860052(9)$ & 0.61258 & $0.1292(6)$ & 0.03 & \\
\hline OGLE-BLG-RRLYR-18350 & $0.29607177(10)$ & $0.181891(1)$ & 0.61435 & $0.1232(8)$ & 0.031 & \\
\hline OGLE-BLG-RRLYR-19952 & $0.2632481(2)$ & $0.1603591(9)$ & 0.60916 & $0.142(2)$ & 0.056 & cand. \\
\hline OGLE-BLG-RRLYR-20257 & $0.31867080(7)$ & $0.201261(1)$ & 0.63156 & $0.1177(4)$ & 0.021 & \\
\hline OGLE-BLG-RRLYR-20374 & $0.35273038(4)$ & $0.215591(2)$ & 0.61121 & $0.1158(7)$ & 0.016 & cand. \\
\hline OGLE-BLG-RRLYR-20431 & $0.33682566(6)$ & $0.206966(1)$ & 0.61446 & $0.1253(4)$ & 0.022 & \\
\hline OGLE-BLG-RRLYR-20565 & $0.30279911(8)$ & $0.185932(1)$ & 0.61404 & $0.1312(6)$ & 0.025 & \\
\hline OGLE-BLG-RRLYR-20633 & $0.3034031(2)$ & $0.185961(2)$ & 0.61292 & $0.127(1)$ & 0.047 & cand. \\
\hline OGLE-BLG-RRLYR-20817 & $0.30788424(6)$ & $0.191525(1)$ & 0.62207 & $0.1310(4)$ & 0.02 & \\
\hline OGLE-BLG-RRLYR-20872 & $0.4358551(1)$ & $0.273852(3)$ & 0.62831 & $0.1140(4)$ & 0.017 & cand. \\
\hline OGLE-BLG-RRLYR-21952 & $0.30652549(7)$ & $0.187470(1)$ & 0.6116 & $0.1373(6)$ & 0.023 & \\
\hline OGLE-BLG-RRLYR-21959 & $0.2663289(2)$ & $0.164554(1)$ & 0.61786 & $0.154(2)$ & 0.097 & bl \\
\hline OGLE-BLG-RRLYR-21968 & $0.30554400(7)$ & $0.187063(1)$ & 0.61223 & $0.1282(5)$ & 0.025 & \\
\hline OGLE-BLG-RRLYR-22388 & $0.30560092(6)$ & $0.187653(1)$ & 0.61405 & $0.1256(4)$ & 0.023 & \\
\hline OGLE-BLG-RRLYR-22492 & $0.26770796(6)$ & $0.163419(1)$ & 0.61044 & $0.1044(5)$ & 0.027 & \\
\hline OGLE-BLG-RRLYR-22667 & $0.3064996(1)$ & $0.188109(1)$ & 0.61373 & $0.1248(7)$ & 0.043 & \\
\hline OGLE-BLG-RRLYR-22808 & $0.29760311(5)$ & $0.1819411(9)$ & 0.61135 & $0.1398(4)$ & 0.024 & \\
\hline OGLE-BLG-RRLYR-22831 & $0.36357282(10)$ & $0.227835(2)$ & 0.62666 & $0.1287(5)$ & 0.015 & cand. \\
\hline OGLE-BLG-RRLYR-22914 & $0.31431319(9)$ & $0.192472(1)$ & 0.61236 & $0.1468(6)$ & 0.014 & cand. \\
\hline OGLE-BLG-RRLYR-23005 & $0.30201733(7)$ & $0.185265(1)$ & 0.61343 & $0.1203(5)$ & 0.027 & \\
\hline OGLE-BLG-RRLYR-23151 & $0.31035740(7)$ & $0.190286(3)$ & 0.61312 & $0.1185(4)$ & 0.024 & \\
\hline OGLE-BLG-RRLYR-23182 & $0.29608550(6)$ & $0.181245(1)$ & 0.61214 & $0.1313(5)$ & 0.021 & \\
\hline OGLE-BLG-RRLYR-23205 & $0.30929644(7)$ & $0.189611(1)$ & 0.61304 & $0.1250(4)$ & 0.024 & \\
\hline OGLE-BLG-RRLYR-23394 & $0.32143411(7)$ & $0.202520(2)$ & 0.63005 & $0.1155(4)$ & 0.016 & cand. \\
\hline OGLE-BLG-RRLYR-23432 & $0.26100392(8)$ & $0.1599070(9)$ & 0.61266 & $0.1066(7)$ & 0.033 & \\
\hline OGLE-BLG-RRLYR-23437 & $0.2873553(3)$ & $0.176546(1)$ & 0.61438 & $0.109(2)$ & 0.092 & cand. \\
\hline OGLE-BLG-RRLYR-23600 & $0.3154509(2)$ & $0.196091(1)$ & 0.62162 & $0.1247(9)$ & 0.038 & \\
\hline OGLE-BLG-RRLYR-23732 & $0.29576237(5)$ & $0.180994(1)$ & 0.61196 & $0.1410(4)$ & 0.015 & cand. \\
\hline OGLE-BLG-RRLYR-23800 & $0.3136775(1)$ & $0.192414(1)$ & 0.61341 & $0.1238(6)$ & 0.03 & \\
\hline OGLE-BLG-RRLYR-24574 & $0.2936512(2)$ & $0.180016(1)$ & 0.61303 & $0.127(1)$ & 0.043 & \\
\hline OGLE-BLG-RRLYR-24936 & $0.3104671(1)$ & $0.190490(2)$ & 0.61356 & $0.1216(6)$ & 0.023 & cand. \\
\hline OGLE-BLG-RRLYR-24945 & $0.29662336(9)$ & $0.182480(1)$ & 0.61519 & $0.1284(6)$ & 0.025 & \\
\hline OGLE-BLG-RRLYR-25450 & $0.3202394(3)$ & $0.1968101(7)$ & 0.61457 & $0.125(1)$ & 0.048 & \\
\hline OGLE-BLG-RRLYR-25727 & $0.295694(1)$ & $0.179973(2)$ & 0.60865 & $0.125(3)$ & 0.16 & cand. \\
\hline OGLE-BLG-RRLYR-25784 & $0.29481845(7)$ & $0.1809363(10)$ & 0.61372 & $0.1365(6)$ & 0.023 & \\
\hline OGLE-BLG-RRLYR-26009 & $0.29079450(7)$ & $0.178454(1)$ & 0.61368 & $0.1394(7)$ & 0.024 & cand. \\
\hline OGLE-BLG-RRLYR-26177 & $0.3188948(3)$ & $0.202164(2)$ & 0.63395 & $0.135(2)$ & 0.074 & cand. \\
\hline
\end{tabular}


Table A1 - Continued from previous page

\begin{tabular}{|c|c|c|c|c|c|c|}
\hline ID & $P_{1 \mathrm{O}}[\mathrm{d}]$ & $P_{\mathrm{X}}[\mathrm{d}]$ & $P_{10} / P_{\mathrm{X}}$ & $A_{10}[\mathrm{mag}]$ & $A_{\mathrm{X}} / A_{1 \mathrm{O}}$ & Remarks \\
\hline OGLE-BLG-RRLYR-26256 & $0.2206397(2)$ & $0.138785(1)$ & 0.62901 & $0.057(1)$ & 0.14 & cand. \\
\hline OGLE-BLG-RRLYR-26263 & $0.2827581(2)$ & $0.174820(1)$ & 0.61827 & $0.126(2)$ & 0.087 & \\
\hline OGLE-BLG-RRLYR-26388 & $0.28715345(9)$ & $0.176726(1)$ & 0.61544 & $0.1377(7)$ & 0.021 & cand. \\
\hline OGLE-BLG-RRLYR-26738 & $0.24384962(7)$ & $0.1495729(7)$ & 0.61338 & $0.1115(7)$ & 0.037 & \\
\hline OGLE-BLG-RRLYR-26891 & $0.30860918(8)$ & $0.189545(1)$ & 0.61419 & $0.1257(5)$ & 0.023 & \\
\hline OGLE-BLG-RRLYR-27690 & $0.23618019(6)$ & $0.1453185(7)$ & 0.61529 & $0.0875(5)$ & 0.03 & \\
\hline OGLE-BLG-RRLYR-27707 & $0.2962165(1)$ & $0.181625(1)$ & 0.61315 & $0.1295(8)$ & 0.032 & cand. \\
\hline OGLE-BLG-RRLYR-28135 & $0.25386558(5)$ & $0.1557970(10)$ & 0.6137 & $0.1049(4)$ & 0.018 & \\
\hline OGLE-BLG-RRLYR-28314 & $0.30371944(5)$ & $0.186405(1)$ & 0.61374 & $0.1019(7)$ & 0.028 & \\
\hline OGLE-BLG-RRLYR-28419 & $0.2890046(1)$ & $0.176978(1)$ & 0.61237 & $0.143(1)$ & 0.035 & cand. \\
\hline OGLE-BLG-RRLYR-29661 & $0.2932706(6)$ & $0.1798607(10)$ & 0.61329 & $0.141(1)$ & 0.02 & \\
\hline OGLE-BLG-RRLYR-29811 & $0.29745601(10)$ & $0.182544(2)$ & 0.61368 & $0.1242(7)$ & 0.023 & cand. \\
\hline OGLE-BLG-RRLYR-30077 & $0.2916725(2)$ & $0.177400(1)$ & 0.60822 & $0.116(1)$ & 0.052 & cand. \\
\hline OGLE-BLG-RRLYR-30249 & $0.2949113(3)$ & $0.182253(1)$ & 0.61799 & $0.143(2)$ & 0.07 & cand. \\
\hline OGLE-BLG-RRLYR-30497 & $0.31193686(9)$ & $0.191383(1)$ & 0.61353 & $0.1314(7)$ & 0.03 & \\
\hline OGLE-BLG-RRLYR-30633 & $0.29149550(5)$ & $0.1789072(9)$ & 0.61376 & $0.1315(4)$ & 0.023 & \\
\hline OGLE-BLG-RRLYR-30707 & $0.3578046(1)$ & $0.224900(2)$ & 0.62856 & $0.1178(5)$ & 0.026 & cand. \\
\hline OGLE-BLG-RRLYR-30713 & $0.3160170(3)$ & $0.191906(1)$ & 0.60726 & $0.113(2)$ & 0.08 & \\
\hline OGLE-BLG-RRLYR-30848 & $0.30692897(4)$ & $0.188418(1)$ & 0.61388 & $0.1235(3)$ & 0.014 & \\
\hline OGLE-BLG-RRLYR-30871 & $0.28671507(9)$ & $0.176257(1)$ & 0.61475 & $0.1454(9)$ & 0.03 & cand. \\
\hline OGLE-BLG-RRLYR-31059 & $0.30336272(4)$ & $0.185668(1)$ & 0.61203 & $0.1405(6)$ & 0.024 & cand. \\
\hline OGLE-BLG-RRLYR-31176 & $0.3012340(1)$ & $0.1849665(9)$ & 0.61403 & $0.1115(7)$ & 0.055 & \\
\hline OGLE-BLG-RRLYR-31457 & $0.25793215(10)$ & $0.1585973(10)$ & 0.61488 & $0.133(1)$ & 0.045 & cand. \\
\hline OGLE-BLG-RRLYR-31479 & $0.25607708(7)$ & $0.1566354(7)$ & 0.61167 & $0.0979(5)$ & 0.031 & \\
\hline OGLE-BLG-RRLYR-31492 & $0.31929505(9)$ & $0.195734(1)$ & 0.61302 & $0.1292(6)$ & 0.021 & cand. \\
\hline OGLE-BLG-RRLYR-31508 & $0.31481230(5)$ & $0.192871(2)$ & 0.61265 & $0.1262(4)$ & 0.011 & cand. \\
\hline OGLE-BLG-RRLYR-31509 & $0.30850728(8)$ & $0.189233(1)$ & 0.61338 & $0.1295(6)$ & 0.022 & cand. \\
\hline OGLE-BLG-RRLYR-31586 & $0.31039365(6)$ & $0.190296(1)$ & 0.61308 & $0.1204(4)$ & 0.017 & cand. \\
\hline OGLE-BLG-RRLYR-31600 & $0.30782878(5)$ & $0.191200(1)$ & 0.62112 & $0.1328(3)$ & 0.016 & $0.5 f_{x}$ \\
\hline OGLE-BLG-RRLYR-31641 & $0.31030202(5)$ & $0.1902879(7)$ & 0.61323 & $0.1288(3)$ & 0.026 & \\
\hline \multirow[t]{2}{*}{ OGLE-BLG-RRLYR-31736 } & $0.30524110(3)$ & $0.187269(3)$ & 0.61351 & $0.1337(2)$ & 0.012 & $0.5 f_{x}$ \\
\hline & $0.30524110(3)$ & $0.1925625(4)$ & 0.63085 & $0.1337(2)$ & 0.011 & \\
\hline OGLE-BLG-RRLYR-31753 & $0.27630146(3)$ & $0.1692550(6)$ & 0.61257 & $0.1133(3)$ & 0.024 & \\
\hline OGLE-BLG-RRLYR-31809 & $0.33831393(7)$ & $0.213062(1)$ & 0.62978 & $0.0878(3)$ & 0.019 & \\
\hline OGLE-BLG-RRLYR-31905 & $0.28806311(4)$ & $0.1768940(9)$ & 0.61408 & $0.1595(4)$ & 0.016 & \\
\hline OGLE-BLG-RRLYR-31950 & $0.28700626(3)$ & $0.1756031(10)$ & 0.61184 & $0.1418(3)$ & 0.013 & \\
\hline OGLE-BLG-RRLYR-31960 & $0.29491649(6)$ & $0.1807751(6)$ & 0.61297 & $0.1354(5)$ & 0.033 & \\
\hline OGLE-BLG-RRLYR-31994 & $0.28845104(6)$ & $0.177339(1)$ & 0.6148 & $0.1030(4)$ & 0.02 & cand. \\
\hline OGLE-BLG-RRLYR-32002 & $0.28662713(5)$ & $0.1757953(5)$ & 0.61332 & $0.1333(4)$ & 0.032 & \\
\hline \multirow[t]{3}{*}{ OGLE-BLG-RRLYR-32054 } & $0.31481782(4)$ & $0.193420(1)$ & 0.61439 & $0.1265(2)$ & 0.012 & \\
\hline & $0.31481782(4)$ & $0.195716(2)$ & 0.62168 & $0.1265(2)$ & 0.009 & \\
\hline & $0.31481782(4)$ & $0.198673(1)$ & 0.63107 & $0.1265(2)$ & 0.01 & \\
\hline OGLE-BLG-RRLYR-32074 & $0.24342902(6)$ & $0.1492172(7)$ & 0.61298 & $0.1029(6)$ & 0.033 & \\
\hline OGLE-BLG-RRLYR-32091 & $0.28894518(2)$ & $0.17690307(9)$ & 0.61224 & $0.1294(2)$ & 0.027 & \\
\hline \multirow{3}{*}{ OGLE-BLG-RRLYR-32139 } & $0.31002046(3)$ & $0.1902447(4)$ & 0.61365 & $0.1275(3)$ & 0.036 & $0.5 f_{x}$ \\
\hline & $0.31002046(3)$ & $0.192991(1)$ & 0.62251 & $0.1275(3)$ & 0.013 & \\
\hline & $0.31002046(3)$ & $0.195867(1)$ & 0.63179 & $0.1275(3)$ & 0.011 & \\
\hline OGLE-BLG-RRLYR-32143 & $0.32123808(4)$ & $0.202574(1)$ & 0.6306 & $0.1225(3)$ & 0.014 & \\
\hline \multirow[t]{2}{*}{ OGLE-BLG-RRLYR-32145 } & $0.30004964(3)$ & $0.1842304(2)$ & 0.614 & $0.1147(2)$ & 0.028 & \\
\hline & $0.30004964(3)$ & $0.186719(3)$ & 0.62229 & $0.1147(2)$ & 0.01 & \\
\hline OGLE-BLG-RRLYR-32151 & $0.32801366(8)$ & $0.201576(1)$ & 0.61454 & $0.1276(6)$ & 0.024 & \\
\hline OGLE-BLG-RRLYR-32158 & $0.30945091(3)$ & $0.189532(1)$ & 0.61248 & $0.1282(3)$ & 0.012 & \\
\hline \multirow[t]{2}{*}{ OGLE-BLG-RRLYR-32180 } & $0.338096820(10)$ & $0.212233(2)$ & 0.62773 & $0.1191(2)$ & 0.009 & $0.5 f_{x}$ \\
\hline & $0.338096820(10)$ & $0.214222(2)$ & 0.63361 & $0.1191(2)$ & 0.008 & \\
\hline OGLE-BLG-RRLYR-32199 & $0.27712149(10)$ & $0.1717888(9)$ & 0.6199 & $0.1148(8)$ & 0.024 & \\
\hline \multirow[t]{3}{*}{ OGLE-BLG-RRLYR-32213 } & $0.24889440(5)$ & $0.1574083(7)$ & 0.63243 & $0.0806(3)$ & 0.03 & \\
\hline & $0.24889440(5)$ & $0.1524801(6)$ & 0.61263 & $0.0806(3)$ & 0.03 & \\
\hline & $0.24889440(5)$ & $0.1574083(7)$ & 0.63243 & $0.0806(3)$ & 0.03 & \\
\hline OGLE-BLG-RRLYR-32252 & $0.36309769(6)$ & $0.228489(1)$ & 0.62928 & $0.1140(2)$ & 0.013 & \\
\hline
\end{tabular}


Table A1 - Continued from previous page

\begin{tabular}{c} 
ID \\
\hline OGLE-BLG-RRLYR-32263 \\
OGLE-BLG-RRLYR-32282 \\
OGLE-BLG-RRLYR-32289 \\
OGLE-BLG-RRLYR-32295 \\
OGLE-BLG-RRLYR-32304 \\
\\
OGLE-BLG-RRLYR-32348 \\
OGLE-BLG-RRLYR-32396 \\
OGLE-BLG-RRLYR-32573 \\
OGLE-BLG-RRLYR-32636 \\
OGLE-BLG-RRLYR-32706 \\
OGLE-BLG-RRLYR-32713 \\
OGLE-BLG-RRLYR-32782 \\
OGLE-BLG-RRLYR-32796 \\
OGLE-BLG-RRLYR-32816 \\
OGLE-BLG-RRLYR-32820 \\
OGLE-BLG-RRLYR-32831 \\
OGLE-BLG-RRLYR-32877
\end{tabular}

OGLE-BLG-RRLYR-33162

OGLE-BLG-RRLYR-33189 OGLE-BLG-RRLYR-33228 OGLE-BLG-RRLYR-33270 OGLE-BLG-RRLYR-33378 OGLE-BLG-RRLYR-33545

OGLE-BLG-RRLYR-33775

OGLE-BLG-RRLYR-33964 OGLE-BLG-RRLYR-34003 OGLE-BLG-RRLYR-34171 OGLE-BLG-RRLYR-34206 OGLE-BLG-RRLYR-34233 OGLE-BLG-RRLYR-34332 OGLE-BLG-RRLYR-34346

OGLE-BLG-RRLYR-34359 OGLE-BLG-RRLYR-34376 OGLE-BLG-RRLYR-34391 OGLE-BLG-RRLYR-34401 OGLE-BLG-RRLYR-34517

OGLE-BLG-RRLYR-34766 OGLE-BLG-RRLYR-34808 OGLE-BLG-RRLYR-35006 OGLE-BLG-RRLYR-35129 OGLE-BLG-RRLYR-35202 OGLE-BLG-RRLYR-35261 OGLE-BLG-RRLYR-35325 OGLE-BLG-RRLYR-35336 OGLE-BLG-RRLYR-35369 OGLE-BLG-RRLYR-35396 OGLE-BLG-RRLYR-35412 OGLE-BLG-RRLYR-35434 OGLE-BLG-RRLYR-35436 OGLE-BLG-RRLYR-35458 OGLE-BLG-RRLYR-35479

\begin{tabular}{|c|c|c|c|c|c|}
\hline$P_{1 \mathrm{O}}[\mathrm{d}]$ & $P_{\mathrm{X}}[\mathrm{d}]$ & $P_{1 \mathrm{O}} / P_{\mathrm{X}}$ & $A_{1 \mathrm{O}}[\mathrm{mag}]$ & $A_{\mathrm{X}} / A_{1 \mathrm{O}}$ & Remarks \\
\hline $0.29433189(5)$ & $0.179923(1)$ & 0.61129 & $0.1332(5)$ & 0.023 & $\mathrm{bl}$ \\
\hline $0.31760989(6)$ & $0.200220(1)$ & 0.63040 & $0.1330(2)$ & 0.013 & $0.5 f_{x}$ \\
\hline $0.30627376(2)$ & $0.1885143(5)$ & 0.61551 & $0.1258(1)$ & 0.015 & \\
\hline $0.31434021(6)$ & $0.199234(2)$ & 0.63382 & $0.1136(3)$ & 0.01 & cand. \\
\hline $0.34573419(7)$ & $0.212025(1)$ & 0.61326 & $0.1317(4)$ & 0.021 & \\
\hline $0.34573419(7)$ & $0.215105(2)$ & 0.62217 & $0.1317(4)$ & 0.017 & \\
\hline $0.34573419(7)$ & $0.218200(2)$ & 0.63112 & $0.1317(4)$ & 0.014 & \\
\hline $0.33697369(5)$ & $0.205291(2)$ & 0.60922 & $0.1342(3)$ & 0.009 & cand. \\
\hline $0.37030911(6)$ & $0.232470(1)$ & 0.62777 & $0.1136(3)$ & 0.017 & $0.5 f_{x}$ \\
\hline $0.33186925(6)$ & $0.208210(2)$ & 0.62739 & $0.1288(4)$ & 0.016 & cand. \\
\hline $0.29001784(7)$ & $0.178090(1)$ & 0.61407 & $0.1339(7)$ & 0.028 & \\
\hline $0.29223304(9)$ & $0.1791172(10)$ & 0.61293 & $0.1281(8)$ & 0.037 & \\
\hline $0.28631104(6)$ & $0.1761364(9)$ & 0.61519 & $0.1710(8)$ & 0.027 & cand. \\
\hline $0.33324349(6)$ & $0.204224(1)$ & 0.61284 & $0.1219(4)$ & 0.018 & \\
\hline $0.21774439(5)$ & $0.1339305(5)$ & 0.61508 & $0.0606(3)$ & 0.033 & \\
\hline $0.31308618(6)$ & $0.194813(1)$ & 0.62223 & $0.1193(4)$ & 0.016 & \\
\hline $0.29418472(5)$ & $0.1802570(9)$ & 0.61273 & $0.1314(5)$ & 0.024 & \\
\hline $0.31124914(9)$ & $0.190610(1)$ & 0.6124 & $0.1229(6)$ & 0.015 & \\
\hline $0.25065626(6)$ & $0.1534253(6)$ & 0.61209 & $0.0731(3)$ & 0.036 & \\
\hline $0.25065626(6)$ & $0.155976(1)$ & 0.62227 & $0.0731(3)$ & 0.022 & \\
\hline $0.23658973(4)$ & $0.1448679(6)$ & 0.61232 & $0.0651(3)$ & 0.038 & \\
\hline $0.23658973(4)$ & $0.1473943(8)$ & 0.623 & $0.0651(3)$ & 0.018 & \\
\hline $0.36514752(9)$ & $0.224241(2)$ & 0.61411 & $0.1512(5)$ & 0.016 & \\
\hline $0.3181641(1)$ & $0.195039(1)$ & 0.61301 & $0.1208(7)$ & 0.022 & cand. \\
\hline $0.3007049(2)$ & $0.184471(1)$ & 0.61346 & $0.128(1)$ & 0.018 & \\
\hline $0.24330738(6)$ & $0.1489998(3)$ & 0.61239 & $0.0810(2)$ & 0.04 & \\
\hline $0.34284320(3)$ & $0.2150169(9)$ & 0.62716 & $0.1216(2)$ & 0.011 & $0.5 f_{x}$ \\
\hline $0.34284320(3)$ & $0.216420(2)$ & 0.63125 & $0.1216(2)$ & 0.009 & \\
\hline $0.24956761(4)$ & $0.1553826(7)$ & 0.62261 & $0.0832(3)$ & 0.024 & $0.5 f_{x}$ \\
\hline $0.24956761(4)$ & $0.1579050(6)$ & 0.63271 & $0.0832(3)$ & 0.018 & \\
\hline $0.21544350(7)$ & $0.1331931(4)$ & 0.61823 & $0.0503(4)$ & 0.095 & bl \\
\hline $0.2664148(2)$ & $0.1689182(5)$ & 0.63404 & $0.0592(9)$ & 0.054 & \\
\hline $0.2845978(2)$ & $0.172472(1)$ & 0.60602 & $0.1139(9)$ & 0.037 & cand. \\
\hline $0.31296335(10)$ & $0.191968(1)$ & 0.61339 & $0.0485(2)$ & 0.023 & \\
\hline $0.30667044(8)$ & $0.188302(1)$ & 0.61402 & $0.1235(5)$ & 0.023 & \\
\hline $0.2971743(2)$ & $0.182503(2)$ & 0.61413 & $0.132(1)$ & 0.02 & \\
\hline $0.26654026(7)$ & $0.1630731(9)$ & 0.61181 & $0.1082(5)$ & 0.026 & cand. \\
\hline $0.26654026(7)$ & $0.1656029(9)$ & 0.62131 & $0.1082(5)$ & 0.025 & \\
\hline $0.29417536(6)$ & $0.180493(1)$ & 0.61356 & $0.1375(5)$ & 0.022 & \\
\hline $0.3096863(1)$ & $0.1895385(10)$ & 0.61203 & $0.1235(6)$ & 0.035 & \\
\hline $0.31664949(6)$ & $0.194074(1)$ & 0.6129 & $0.1251(3)$ & 0.017 & $0.5 f_{x}$ \\
\hline $0.27249053(2)$ & $0.167629(6)$ & 0.61517 & $0.1505(2)$ & 0.008 & cand. \\
\hline $0.31834976(6)$ & $0.197895(1)$ & 0.62163 & $0.1216(3)$ & 0.012 & \\
\hline $0.31834976(6)$ & $0.195074(2)$ & 0.61277 & $0.1216(3)$ & 0.012 & \\
\hline $0.3102331(3)$ & $0.189451(2)$ & 0.61067 & $0.123(2)$ & 0.065 & cand. \\
\hline $0.31487440(6)$ & $0.1934919(10)$ & 0.61451 & $0.1248(4)$ & 0.02 & \\
\hline $0.29745(3)$ & $0.1827037(9)$ & 0.61423 & $0.13(2)$ & 0.026 & \\
\hline $0.25505101(3)$ & $0.1562090(8)$ & 0.61246 & $0.1031(5)$ & 0.034 & \\
\hline $0.25138603(5)$ & $0.1540225(7)$ & 0.61269 & $0.0856(3)$ & 0.022 & \\
\hline $0.31208884(10)$ & $0.191734(3)$ & 0.61436 & $0.1333(6)$ & 0.025 & \\
\hline $0.30297353(7)$ & $0.186059(1)$ & 0.61411 & $0.1251(5)$ & 0.018 & \\
\hline $0.28635102(5)$ & $0.175925(1)$ & 0.61437 & $0.1293(4)$ & 0.018 & \\
\hline $0.28166515(2)$ & $0.1732000(6)$ & 0.61491 & $0.1460(3)$ & 0.016 & \\
\hline $0.30821088(7)$ & $0.1891652(7)$ & 0.61375 & $0.1212(5)$ & 0.032 & \\
\hline $0.30144849(8)$ & $0.185196(1)$ & 0.61435 & $0.1322(6)$ & 0.023 & \\
\hline $0.23154470(3)$ & $0.1421632(2)$ & 0.61398 & $0.1141(4)$ & 0.017 & \\
\hline $0.23590825(4)$ & $0.1445258(5)$ & 0.61264 & $0.0801(3)$ & 0.03 & \\
\hline $0.31163149(8)$ & $0.1909877(9)$ & 0.61286 & $0.1136(6)$ & 0.031 & \\
\hline $0.310921(2)$ & $0.190720(1)$ & 0.6134 & $0.119(1)$ & 0.026 & cand. \\
\hline
\end{tabular}


Table A1 - Continued from previous page

$$
\text { ID }
$$

OGLE-BLG-RRLYR-35524

OGLE-BLG-RRLYR-35665

OGLE-BLG-RRLYR-35788

OGLE-BLG-RRLYR-35820

OGLE-BLG-RRLYR-35864

OGLE-BLG-RRLYR-36146

OGLE-BLG-RRLYR-36165

OGLE-BLG-RRLYR-36173

OGLE-BLG-RRLYR-36303

OGLE-BLG-RRLYR-36522

OGLE-BLG-RRLYR-36685

OGLE-BLG-RRLYR-38634

OGLE-BLG-RRLYR-38729

OGLE-BLG-RRLYR-38777

OGLE-BLG-RRLYR-39022

$P_{10}[\mathrm{~d}]$

$0.28133186(6)$

$0.31548138(7)$

$0.28865510(6)$

$0.23863501(7)$

$0.28760561(7)$

$0.3076168(1)$

$0.31859264(8)$

$0.29459646(7)$

$0.30359726(8)$

$0.35432453(8)$

$0.2891668(2)$

$0.2382020(1)$

$0.2250438(1)$

$0.2074844(1)$

$0.3687967(2)$

$P_{\mathrm{X}}[\mathrm{d}]$

$0.173036(1)$

$0.193523(1)$

$0.1774204(10)$

$0.1464143(8)$

$0.176621(1)$

$0.188724(1)$

$0.201045(1)$

$0.181036(1)$

$0.186170(1)$

$0.216845(2)$

$0.177459(2)$

$0.1462079(8)$

$0.1393534(6)$

$0.1290387(4)$

$0.232673(2)$
$P_{1 \mathrm{O}} / P_{\mathrm{X}} \quad A_{1 \mathrm{O}}[\mathrm{mag}]$ $A_{\mathrm{X}} / A_{1 \mathrm{O}} \quad$ Remarks $\begin{array}{lll}0.61506 & 0.1494(5) & 0.019\end{array}$

$\begin{array}{lll}0.61342 & 0.1282(5) & 0.019\end{array}$

$\begin{array}{lll}0.61464 & 0.1346(5) & 0.022\end{array}$

$\begin{array}{lll}0.61355 & 0.1141(7) & 0.046\end{array}$

$\begin{array}{lll}0.61411 & 0.1334(6) & 0.02\end{array}$

$\begin{array}{lll}0.6135 & 0.1300(8) & 0.032\end{array}$

$\begin{array}{lll}0.63104 & 0.1391(6) & 0.026\end{array}$

$\begin{array}{lll}0.61452 & 0.1346(5) & 0.023\end{array}$

$\begin{array}{lll}0.61321 & 0.1264(5) & 0.023\end{array}$

$\begin{array}{lll}0.612 & 0.1359(6) & 0.015\end{array}$

$\begin{array}{lll}0.61369 & 0.1378(8) & 0.033\end{array}$

$\begin{array}{lll}0.6138 & 0.0744(8) & 0.055\end{array}$

$\begin{array}{lll}0.61923 & 0.0328(4) & 0.079\end{array}$

$\begin{array}{lll}0.62192 & 0.0403(7) & 0.206\end{array}$

$0.6309 \quad 0.0858(5) \quad 0.016 \quad$ cand. 
Table A2: Properties of $\mathrm{RR}_{0.68}$ stars. Consecutive columns provide name of a star, its first-overtone period, period of the additional signal, ratio between the two periods, amplitude of the first overtone and ratio of amplitude of the additional signal to the amplitude of the first overtone mode. Last column contains remarks on individual stars: 'comb.' - there is a combination between the first overtone and the additional signal, 'bl' - Blazhko effect is present in the star.

\begin{tabular}{|c|c|c|c|c|c|c|}
\hline ID & $P_{1 \mathrm{O}}[\mathrm{d}]$ & $P_{\mathrm{X}}[\mathrm{d}]$ & $P_{\mathrm{X}} / P_{1 \mathrm{O}}$ & $A_{1 \mathrm{O}}[\mathrm{mag}]$ & $A_{\mathrm{X}} / A_{1 \mathrm{O}}$ & Remarks \\
\hline OGLE-BLG-RRLYR-01064 & $0.31486155(4)$ & $0.458607(6)$ & 0.68656 & $0.1239(3)$ & 0.016 & \\
\hline OGLE-BLG-RRLYR-01152 & $0.34759513(10)$ & $0.507535(4)$ & 0.68487 & $0.1084(5)$ & 0.04 & comb. \\
\hline OGLE-BLG-RRLYR-01408 & $0.3853534(3)$ & $0.56176(1)$ & 0.68598 & $0.112(1)$ & 0.04 & \\
\hline OGLE-BLG-RRLYR-01436 & $0.3242810(5)$ & $0.47942(2)$ & 0.6764 & $0.120(3)$ & 0.04 & \\
\hline OGLE-BLG-RRLYR-01808 & $0.3161546(2)$ & $0.460225(5)$ & 0.68696 & $0.0937(8)$ & 0.063 & \\
\hline OGLE-BLG-RRLYR-03053 & $0.2898407(1)$ & $0.423478(6)$ & 0.68443 & $0.1273(9)$ & 0.042 & $\mathrm{bl}$ \\
\hline OGLE-BLG-RRLYR-03058 & $0.3862909(2)$ & $0.56395(1)$ & 0.68497 & $0.1342(9)$ & 0.019 & $\mathrm{bl}$ \\
\hline OGLE-BLG-RRLYR-03581 & $0.3763837(3)$ & $0.549241(5)$ & 0.68528 & $0.0964(9)$ & 0.059 & \\
\hline OGLE-BLG-RRLYR-04192 & $0.3350892(1)$ & $0.489131(7)$ & 0.68507 & $0.1101(5)$ & 0.03 & \\
\hline OGLE-BLG-RRLYR-04264 & $0.25384340(4)$ & $0.371494(1)$ & 0.6833 & $0.1166(3)$ & 0.021 & $\mathrm{bl}$ \\
\hline OGLE-BLG-RRLYR-04506 & $0.27507883(3)$ & $0.403839(6)$ & 0.68116 & $0.1526(3)$ & 0.013 & \\
\hline OGLE-BLG-RRLYR-04714 & $0.28915072(10)$ & $0.421093(7)$ & 0.68667 & $0.1189(8)$ & 0.02 & \\
\hline OGLE-BLG-RRLYR-04994 & $0.3623021(1)$ & $0.527853(6)$ & 0.68637 & $0.0802(3)$ & 0.039 & \\
\hline OGLE-BLG-RRLYR-05080 & $0.29969806(8)$ & $0.436809(2)$ & 0.68611 & $0.1358(3)$ & 0.046 & comb. \\
\hline OGLE-BLG-RRLYR-05428 & $0.35477206(5)$ & $0.517599(5)$ & 0.68542 & $0.1290(2)$ & 0.019 & \\
\hline OGLE-BLG-RRLYR-05709 & $0.4102718(2)$ & $0.600101(4)$ & 0.68367 & $0.0844(4)$ & 0.062 & comb. \\
\hline OGLE-BLG-RRLYR-06402 & $0.40747615(7)$ & $0.593479(9)$ & 0.68659 & $0.1086(2)$ & 0.015 & \\
\hline OGLE-BLG-RRLYR-06446 & $0.35821090(7)$ & $0.515816(3)$ & 0.69445 & $0.1111(3)$ & 0.034 & \\
\hline OGLE-BLG-RRLYR-06754 & $0.3530891(2)$ & $0.513926(7)$ & 0.68704 & $0.1113(7)$ & 0.022 & \\
\hline OGLE-BLG-RRLYR-06791 & $0.31127928(5)$ & $0.459519(4)$ & 0.6774 & $0.0790(2)$ & 0.028 & \\
\hline OGLE-BLG-RRLYR-06913 & $0.30005824(3)$ & $0.437305(4)$ & 0.68615 & $0.1243(7)$ & 0.027 & comb. \\
\hline OGLE-BLG-RRLYR-06963 & $0.3701000(3)$ & $0.540224(5)$ & 0.68509 & $0.120(1)$ & 0.028 & \\
\hline OGLE-BLG-RRLYR-06970 & $0.42988951(2)$ & $0.626501(4)$ & 0.68618 & $0.12694(8)$ & 0.012 & \\
\hline OGLE-BLG-RRLYR-07054 & $0.3522689(1)$ & $0.524518(6)$ & 0.6716 & $0.1078(5)$ & 0.019 & \\
\hline OGLE-BLG-RRLYR-07127 & $0.377884(3)$ & $0.550563(7)$ & 0.68636 & $0.103(5)$ & 0.032 & \\
\hline OGLE-BLG-RRLYR-07428 & $0.3268696(1)$ & $0.477291(7)$ & 0.68484 & $0.1123(5)$ & 0.026 & \\
\hline OGLE-BLG-RRLYR-07653 & $0.31117973(5)$ & $0.454155(2)$ & 0.68518 & $0.1133(3)$ & 0.025 & $\mathrm{bl}$ \\
\hline OGLE-BLG-RRLYR-07673 & $0.36628701(5)$ & $0.534252(1)$ & 0.68561 & $0.1165(2)$ & 0.06 & \\
\hline OGLE-BLG-RRLYR-07795 & $0.28498705(10)$ & $0.416035(5)$ & 0.68501 & $0.1118(7)$ & 0.018 & \\
\hline OGLE-BLG-RRLYR-08006 & $0.3005622(2)$ & $0.438004(5)$ & 0.68621 & $0.148(2)$ & 0.049 & comb. \\
\hline OGLE-BLG-RRLYR-08076 & $0.28448241(4)$ & $0.4153347(7)$ & 0.68495 & $0.1353(3)$ & 0.1 & comb. \\
\hline OGLE-BLG-RRLYR-08243 & $0.32507556(10)$ & $0.473290(7)$ & 0.68684 & $0.1098(5)$ & 0.018 & \\
\hline OGLE-BLG-RRLYR-08466 & $0.3015875(2)$ & $0.439480(6)$ & 0.68624 & $0.072(2)$ & 0.057 & \\
\hline OGLE-BLG-RRLYR-08680 & $0.34769682(6)$ & $0.505277(3)$ & 0.68813 & $0.1366(4)$ & 0.015 & \\
\hline OGLE-BLG-RRLYR-08748 & $0.29154582(4)$ & $0.424904(1)$ & 0.68615 & $0.1172(3)$ & 0.039 & \\
\hline OGLE-BLG-RRLYR-08808 & $0.34781176(10)$ & $0.507064(6)$ & 0.68593 & $0.1324(6)$ & 0.031 & \\
\hline OGLE-BLG-RRLYR-08832 & $0.30360473(4)$ & $0.441876(3)$ & 0.68708 & $0.1117(3)$ & 0.03 & $\mathrm{bl}$ \\
\hline OGLE-BLG-RRLYR-08879 & $0.32433994(5)$ & $0.468103(3)$ & 0.69288 & $0.1158(2)$ & 0.015 & comb. \\
\hline OGLE-BLG-RRLYR-08884 & $0.30405745(6)$ & $0.442167(3)$ & 0.68765 & $0.1306(4)$ & 0.035 & \\
\hline OGLE-BLG-RRLYR-09146 & $0.35215975(2)$ & $0.512426(1)$ & 0.68724 & $0.1479(1)$ & 0.028 & comb. \\
\hline OGLE-BLG-RRLYR-09152 & $0.29421007(6)$ & $0.428739(3)$ & 0.68622 & $0.1413(6)$ & 0.028 & comb. \\
\hline OGLE-BLG-RRLYR-09217 & $0.29391373(2)$ & $0.428721(2)$ & 0.68556 & $0.1077(1)$ & 0.024 & \\
\hline OGLE-BLG-RRLYR-09315 & $0.35667080(6)$ & $0.520653(9)$ & 0.68505 & $0.1093(2)$ & 0.012 & \\
\hline OGLE-BLG-RRLYR-09323 & $0.32622775(6)$ & $0.475398(5)$ & 0.68622 & $0.1171(4)$ & 0.027 & \\
\hline OGLE-BLG-RRLYR-09426 & $0.22339317(2)$ & $0.325072(2)$ & 0.68721 & $0.0715(1)$ & 0.017 & \\
\hline OGLE-BLG-RRLYR-09471 & $0.27545071(4)$ & $0.408273(4)$ & 0.67467 & $0.1207(3)$ & 0.039 & comb. \\
\hline OGLE-BLG-RRLYR-09516 & $0.40034558(7)$ & $0.583925(6)$ & 0.68561 & $0.1208(3)$ & 0.017 & \\
\hline OGLE-BLG-RRLYR-09671 & $0.28267064(2)$ & $0.4141634(9)$ & 0.68251 & $0.1536(2)$ & 0.02 & comb. \\
\hline OGLE-BLG-RRLYR-09684 & $0.30376835(8)$ & $0.443086(7)$ & 0.68557 & $0.0900(4)$ & 0.02 & \\
\hline OGLE-BLG-RRLYR-09752 & $0.33273947(7)$ & $0.485611(2)$ & 0.6852 & $0.1345(2)$ & 0.026 & \\
\hline OGLE-BLG-RRLYR-09788 & $0.2821596(1)$ & $0.410939(4)$ & 0.68662 & $0.137(1)$ & 0.019 & \\
\hline OGLE-BLG-RRLYR-09921 & $0.3249314(1)$ & $0.476746(7)$ & 0.68156 & $0.1248(8)$ & 0.038 & \\
\hline
\end{tabular}


Table A2 - Continued from previous page

\begin{tabular}{|c|c|c|c|c|c|c|}
\hline ID & $P_{1 \mathrm{O}}[\mathrm{d}]$ & $P_{\mathrm{X}}[\mathrm{d}]$ & $P_{1 \mathrm{O}} / P_{\mathrm{X}}$ & $A_{10}[\mathrm{mag}]$ & $A_{\mathrm{X}} / A_{1 \mathrm{O}}$ & Remarks \\
\hline OGLE-BLG-RRLYR-09998 & $0.3603170(4)$ & $0.525014(2)$ & 0.6863 & $0.0748(9)$ & 0.025 & \\
\hline OGLE-BLG-RRLYR-10100 & $0.4174338(3)$ & $0.609478(1)$ & 0.6849 & $0.0732(6)$ & 0.094 & \\
\hline OGLE-BLG-RRLYR-10153 & $0.34635591(3)$ & $0.505007(3)$ & 0.68584 & $0.1439(2)$ & 0.015 & \\
\hline OGLE-BLG-RRLYR-10318 & $0.36481785(5)$ & $0.533031(5)$ & 0.68442 & $0.0956(2)$ & 0.021 & \\
\hline OGLE-BLG-RRLYR-10390 & $0.28294441(10)$ & $0.411908(3)$ & 0.68691 & $0.1215(7)$ & 0.019 & \\
\hline OGLE-BLG-RRLYR-10401 & $0.27062947(3)$ & $0.394159(4)$ & 0.6866 & $0.1278(3)$ & 0.016 & comb. \\
\hline OGLE-BLG-RRLYR-10427 & $0.34424092(4)$ & $0.507088(7)$ & 0.67886 & $0.1064(2)$ & 0.011 & comb. \\
\hline OGLE-BLG-RRLYR-10474 & $0.28901302(3)$ & $0.417259(6)$ & 0.69265 & $0.1378(3)$ & 0.011 & \\
\hline OGLE-BLG-RRLYR-10537 & $0.3665323(3)$ & $0.535687(5)$ & 0.68423 & $0.0986(9)$ & 0.047 & \\
\hline OGLE-BLG-RRLYR-10766 & $0.31436187(3)$ & $0.456659(5)$ & 0.6884 & $0.1093(1)$ & 0.009 & \\
\hline OGLE-BLG-RRLYR-10836 & $0.28888881(2)$ & $0.422426(2)$ & 0.68388 & $0.1061(1)$ & 0.027 & bl \\
\hline OGLE-BLG-RRLYR-10924 & $0.39507662(4)$ & $0.575102(5)$ & 0.68697 & $0.1278(1)$ & 0.011 & \\
\hline OGLE-BLG-RRLYR-11091 & $0.3641257(1)$ & $0.531420(5)$ & 0.68519 & $0.1306(6)$ & 0.019 & \\
\hline OGLE-BLG-RRLYR-11099 & $0.2932960(1)$ & $0.422374(4)$ & 0.6944 & $0.132(1)$ & 0.023 & \\
\hline OGLE-BLG-RRLYR-11167 & $0.26610202(3)$ & $0.389773(2)$ & 0.68271 & $0.0859(1)$ & 0.026 & $\mathrm{bl}$ \\
\hline OGLE-BLG-RRLYR-11332 & $0.27560220(6)$ & $0.401585(4)$ & 0.68629 & $0.1260(5)$ & 0.025 & \\
\hline OGLE-BLG-RRLYR-11457 & $0.31576255(2)$ & $0.460148(5)$ & 0.68622 & $0.1402(1)$ & 0.006 & \\
\hline OGLE-BLG-RRLYR-11508 & $0.29317212(2)$ & $0.4289023(10)$ & 0.68354 & $0.1086(1)$ & 0.025 & bl \\
\hline OGLE-BLG-RRLYR-11573 & $0.3531318(1)$ & $0.516871(7)$ & 0.68321 & $0.1203(5)$ & 0.017 & \\
\hline OGLE-BLG-RRLYR-11615 & $0.36799364(4)$ & $0.534057(5)$ & 0.68905 & $0.1596(2)$ & 0.013 & \\
\hline OGLE-BLG-RRLYR-11651 & $0.28954120(5)$ & $0.422708(6)$ & 0.68497 & $0.1430(5)$ & 0.016 & \\
\hline OGLE-BLG-RRLYR-11719 & $0.2944020(1)$ & $0.430238(5)$ & 0.68428 & $0.1327(9)$ & 0.017 & \\
\hline OGLE-BLG-RRLYR-11753 & $0.25910064(2)$ & $0.375306(1)$ & 0.69037 & $0.0907(1)$ & 0.03 & bl \\
\hline OGLE-BLG-RRLYR-11780 & $0.30307160(5)$ & $0.444715(2)$ & 0.6815 & $0.0581(2)$ & 0.045 & \\
\hline OGLE-BLG-RRLYR-11856 & $0.29625757(4)$ & $0.431400(2)$ & 0.68674 & $0.1365(3)$ & 0.025 & \\
\hline OGLE-BLG-RRLYR-11875 & $0.3468205(1)$ & $0.506607(3)$ & 0.68459 & $0.0714(3)$ & 0.064 & \\
\hline OGLE-BLG-RRLYR-11977 & $0.3591727(3)$ & $0.524350(4)$ & 0.68499 & $0.0919(8)$ & 0.025 & \\
\hline OGLE-BLG-RRLYR-12014 & $0.3549333(2)$ & $0.520279(6)$ & 0.6822 & $0.1290(9)$ & 0.02 & \\
\hline OGLE-BLG-RRLYR-12086 & $0.27799888(7)$ & $0.411307(5)$ & 0.67589 & $0.1343(6)$ & 0.028 & \\
\hline OGLE-BLG-RRLYR-12096 & $0.36764697(6)$ & $0.535590(7)$ & 0.68643 & $0.1088(4)$ & 0.027 & \\
\hline OGLE-BLG-RRLYR-12218 & $0.3508042(1)$ & $0.510965(2)$ & 0.68655 & $0.1397(9)$ & 0.009 & \\
\hline OGLE-BLG-RRLYR-12297 & $0.3218767(1)$ & $0.470563(6)$ & 0.68402 & $0.1007(6)$ & 0.021 & \\
\hline OGLE-BLG-RRLYR-12448 & $0.31994492(8)$ & $0.466865(5)$ & 0.68531 & $0.0502(2)$ & 0.024 & \\
\hline OGLE-BLG-RRLYR-12659 & $0.2644574(1)$ & $0.386308(2)$ & 0.68458 & $0.0780(8)$ & 0.067 & bl \\
\hline OGLE-BLG-RRLYR-13148 & $0.31094608(3)$ & $0.454121(2)$ & 0.68472 & $0.1215(1)$ & 0.029 & $\mathrm{bl}$ \\
\hline OGLE-BLG-RRLYR-13364 & $0.32131868(9)$ & $0.468434(5)$ & 0.68594 & $0.1161(4)$ & 0.028 & \\
\hline OGLE-BLG-RRLYR-13470 & $0.3636541(1)$ & $0.5273196(2)$ & 0.68963 & $0.1051(6)$ & 0.239 & bl \\
\hline OGLE-BLG-RRLYR-13473 & $0.2841456(2)$ & $0.414489(1)$ & 0.68553 & $0.0885(10)$ & 0.054 & comb. \\
\hline OGLE-BLG-RRLYR-13649 & $0.28090957(3)$ & $0.413852(3)$ & 0.67877 & $0.1382(4)$ & 0.024 & \\
\hline OGLE-BLG-RRLYR-13946 & $0.3659967(3)$ & $0.533990(7)$ & 0.6854 & $0.0667(6)$ & 0.066 & \\
\hline OGLE-BLG-RRLYR-14175 & $0.34508350(6)$ & $0.508744(6)$ & 0.6783 & $0.1303(3)$ & 0.032 & \\
\hline OGLE-BLG-RRLYR-14249 & $0.3271560(2)$ & $0.477165(5)$ & 0.68562 & $0.0838(8)$ & 0.041 & \\
\hline OGLE-BLG-RRLYR-14509 & $0.3223140(1)$ & $0.467379(7)$ & 0.68962 & $0.1207(6)$ & 0.03 & \\
\hline OGLE-BLG-RRLYR-14576 & $0.35193153(9)$ & $0.513251(7)$ & 0.68569 & $0.1373(5)$ & 0.02 & \\
\hline OGLE-BLG-RRLYR-14603 & $0.35182806(9)$ & $0.513162(3)$ & 0.68561 & $0.1276(5)$ & 0.024 & \\
\hline OGLE-BLG-RRLYR-14643 & $0.31803437(6)$ & $0.464795(6)$ & 0.68425 & $0.1082(3)$ & 0.028 & $\mathrm{bl}$ \\
\hline OGLE-BLG-RRLYR-14712 & $0.3159050(1)$ & $0.457925(3)$ & 0.68986 & $0.1149(8)$ & 0.028 & \\
\hline OGLE-BLG-RRLYR-14775 & $0.33467567(6)$ & $0.486812(4)$ & 0.68748 & $0.1374(4)$ & 0.02 & comb. \\
\hline OGLE-BLG-RRLYR-14793 & $0.29167885(3)$ & $0.42505(2)$ & 0.68622 & $0.1388(6)$ & 0.015 & \\
\hline OGLE-BLG-RRLYR-15292 & $0.31023726(7)$ & $0.452367(5)$ & 0.68581 & $0.1097(4)$ & 0.025 & \\
\hline OGLE-BLG-RRLYR-15306 & $0.29835917(5)$ & $0.434121(4)$ & 0.68727 & $0.1089(3)$ & 0.025 & comb. \\
\hline OGLE-BLG-RRLYR-15316 & $0.30598042(5)$ & $0.445961(5)$ & 0.68611 & $0.1246(4)$ & 0.025 & $\mathrm{bl}$ \\
\hline OGLE-BLG-RRLYR-15453 & $0.3120852(1)$ & $0.451768(5)$ & 0.69081 & $0.0793(5)$ & 0.043 & \\
\hline OGLE-BLG-RRLYR-18477 & $0.2735329(1)$ & $0.399581(6)$ & 0.68455 & $0.1103(9)$ & 0.039 & \\
\hline OGLE-BLG-RRLYR-20374 & $0.35273038(4)$ & $0.513955(4)$ & 0.68631 & $0.1158(7)$ & 0.035 & \\
\hline OGLE-BLG-RRLYR-20527 & $0.3893246(2)$ & $0.567486(6)$ & 0.68605 & $0.1019(6)$ & 0.046 & bl \\
\hline OGLE-BLG-RRLYR-21483 & $0.4232227(2)$ & $0.61761(1)$ & 0.68526 & $0.1112(7)$ & 0.014 & \\
\hline OGLE-BLG-RRLYR-23400 & $0.29065598(4)$ & $0.427389(4)$ & 0.68007 & $0.1287(3)$ & 0.025 & \\
\hline OGLE-BLG-RRLYR-23539 & $0.2641039(3)$ & $0.388081(6)$ & 0.68054 & $0.123(2)$ & 0.089 & \\
\hline
\end{tabular}


Table A2 - Continued from previous page

\begin{tabular}{|c|c|c|c|c|c|c|}
\hline ID & $P_{1 \mathrm{O}}[\mathrm{d}]$ & $P_{\mathrm{X}}[\mathrm{d}]$ & $P_{1 \mathrm{O}} / P_{\mathrm{X}}$ & $A_{10}[\mathrm{mag}]$ & $A_{\mathrm{X}} / A_{1 \mathrm{O}}$ & Remarks \\
\hline OGLE-BLG-RRLYR-23583 & $0.3729788(4)$ & $0.544064(9)$ & 0.68554 & $0.092(1)$ & 0.087 & $\mathrm{bl}$ \\
\hline OGLE-BLG-RRLYR-24161 & $0.35717771(10)$ & $0.520925(9)$ & 0.68566 & $0.1333(6)$ & 0.02 & \\
\hline OGLE-BLG-RRLYR-24204 & $0.27472585(6)$ & $0.397967(4)$ & 0.69032 & $0.1406(6)$ & 0.031 & \\
\hline OGLE-BLG-RRLYR-25192 & $0.27129879(9)$ & $0.398920(3)$ & 0.68008 & $0.0978(6)$ & 0.052 & \\
\hline OGLE-BLG-RRLYR-25498 & $0.26460343(8)$ & $0.385529(4)$ & 0.68634 & $0.1326(9)$ & 0.038 & \\
\hline OGLE-BLG-RRLYR-26809 & $0.26095299(8)$ & $0.380805(3)$ & 0.68527 & $0.0971(6)$ & 0.045 & \\
\hline OGLE-BLG-RRLYR-27023 & $0.3172598(2)$ & $0.465487(9)$ & 0.68157 & $0.142(1)$ & 0.038 & \\
\hline OGLE-BLG-RRLYR-28293 & $0.3684074(3)$ & $0.537252(3)$ & 0.68573 & $0.124(2)$ & 0.029 & \\
\hline OGLE-BLG-RRLYR-28763 & $0.3518545(3)$ & $0.512690(4)$ & 0.68629 & $0.106(1)$ & 0.024 & \\
\hline OGLE-BLG-RRLYR-29582 & $0.3053653(4)$ & $0.44747(1)$ & 0.68243 & $0.109(1)$ & 0.083 & \\
\hline OGLE-BLG-RRLYR-30601 & $0.28369900(7)$ & $0.412857(2)$ & 0.68716 & $0.1155(5)$ & 0.076 & comb. \\
\hline OGLE-BLG-RRLYR-30913 & $0.3601654(3)$ & $0.526911(7)$ & 0.68354 & $0.0850(9)$ & 0.052 & \\
\hline OGLE-BLG-RRLYR-31374 & $0.31427292(4)$ & $0.465617(2)$ & 0.67496 & $0.1104(2)$ & 0.041 & \\
\hline OGLE-BLG-RRLYR-31675 & $0.28193075(4)$ & $0.411128(4)$ & 0.68575 & $0.1499(5)$ & 0.021 & \\
\hline OGLE-BLG-RRLYR-31689 & $0.37833658(5)$ & $0.55111(1)$ & 0.6865 & $0.1416(4)$ & 0.016 & \\
\hline OGLE-BLG-RRLYR-31756 & $0.29651674(3)$ & $0.431651(2)$ & 0.68694 & $0.1389(3)$ & 0.028 & comb. \\
\hline OGLE-BLG-RRLYR-32193 & $0.3072905(1)$ & $0.454946(6)$ & 0.67544 & $0.0764(4)$ & 0.033 & \\
\hline OGLE-BLG-RRLYR-32196 & $0.26774759(5)$ & $0.390688(3)$ & 0.68532 & $0.1000(3)$ & 0.033 & $\mathrm{bl}$ \\
\hline OGLE-BLG-RRLYR-32230 & $0.35933125(7)$ & $0.526130(8)$ & 0.68297 & $0.1300(4)$ & 0.017 & \\
\hline OGLE-BLG-RRLYR-32334 & $0.3930328(3)$ & $0.573630(4)$ & 0.68517 & $0.0737(7)$ & 0.05 & \\
\hline OGLE-BLG-RRLYR-32789 & $0.34975545(8)$ & $0.510378(4)$ & 0.68529 & $0.0920(3)$ & 0.039 & $\mathrm{bl}$ \\
\hline OGLE-BLG-RRLYR-33635 & $0.28068403(4)$ & $0.409564(2)$ & 0.68532 & $0.0462(1)$ & 0.044 & \\
\hline OGLE-BLG-RRLYR-33638 & $0.35814586(5)$ & $0.52441(2)$ & 0.68295 & $0.0624(1)$ & 0.021 & $\mathrm{bl}$ \\
\hline OGLE-BLG-RRLYR-33695 & $0.3602471(2)$ & $0.525550(6)$ & 0.68547 & $0.0800(6)$ & 0.046 & $\mathrm{bl}$ \\
\hline OGLE-BLG-RRLYR-33903 & $0.2822250(2)$ & $0.414125(10)$ & 0.6815 & $0.0800(4)$ & 0.031 & \\
\hline OGLE-BLG-RRLYR-33993 & $0.3710790(1)$ & $0.540394(8)$ & 0.68668 & $0.1273(6)$ & 0.02 & \\
\hline OGLE-BLG-RRLYR-34106 & $0.31537940(10)$ & $0.455995(8)$ & 0.69163 & $0.1273(6)$ & 0.021 & \\
\hline OGLE-BLG-RRLYR-34273 & $0.28007257(2)$ & $0.408058(6)$ & 0.68635 & $0.1208(6)$ & 0.021 & \\
\hline OGLE-BLG-RRLYR-34336 & $0.4250681(6)$ & $0.619784(4)$ & 0.68583 & $0.080(1)$ & 0.028 & \\
\hline OGLE-BLG-RRLYR-34540 & $0.27718010(4)$ & $0.406985(2)$ & 0.68106 & $0.1393(4)$ & 0.035 & $\mathrm{bl}$ \\
\hline OGLE-BLG-RRLYR-35159 & $0.30767254(7)$ & $0.448514(5)$ & 0.68598 & $0.1019(4)$ & 0.021 & \\
\hline OGLE-BLG-RRLYR-35328 & $0.30681327(7)$ & $0.445975(4)$ & 0.68796 & $0.1257(4)$ & 0.022 & $\mathrm{bl}$ \\
\hline OGLE-BLG-RRLYR-35896 & $0.3231642(1)$ & $0.473267(2)$ & 0.68284 & $0.1273(7)$ & 0.031 & \\
\hline OGLE-BLG-RRLYR-36049 & $0.3574694(3)$ & $0.521800(9)$ & 0.68507 & $0.0886(9)$ & 0.053 & $\mathrm{bl}$ \\
\hline OGLE-BLG-RRLYR-36404 & $0.28782534(10)$ & $0.420318(6)$ & 0.68478 & $0.1399(9)$ & 0.035 & \\
\hline OGLE-BLG-RRLYR-36675 & $0.3271381(1)$ & $0.479327(8)$ & 0.68249 & $0.1324(5)$ & 0.042 & \\
\hline
\end{tabular}

This paper has been typeset from a $\mathrm{TE}_{\mathrm{E}} \mathrm{L} / \mathrm{L} \mathrm{T} \mathrm{E}$ file prepared by the author. 